\title{
Abstracts for the 34th HGSA Annual Scientific Meeting, Melbourne, Australia November 16-18, 2010
}

\section{Invited Abstracts}

\section{HEMATOPOIETIC STEM CELL GENE THERAPY WITH LENTIVIRAL VECTOR IN X-LINKED ADRENOLEUKODYSTROPHY}

P.Aubourg ${ }^{1,2}$, S. Hacein-Bey-Abina ${ }^{3}$, C. Bartholomä4 ${ }^{4}$ M. Schmidt ${ }^{4}$, I. Kutschera ${ }^{4}$, M.Vidaud', L. Dal-Cortivo ${ }^{3}$, N. Malhaoui', C. Bellesme' ${ }^{2}$, F. Lefrère ${ }^{3}$, B. L'Homme', P. Bougnères ${ }^{2}$, A. Fischer ${ }^{5}$, M. Cavazzana-Calvo ${ }^{3}$, N. Cartier ${ }^{1,2}$

'University Paris-Descartes, Inserm UMR745, Paris, France

${ }^{2}$ Hôpital Saint-Vincent de Paul, Department of Pediatric Endocrinology and Neurology, Paris, France

${ }^{3}$ Hôpital Necker-Enfants Malades, Department of Biotherapy, Paris, France ${ }^{4}$ DKFZ, National Center for Tumor Diseases and German Cancer Research Center Heidelberg, Germany

${ }^{5}$ Hôpital Necker-Enfants Malades, Department of Pediatric Immuno-Hematology, Paris, France

$\mathrm{X}$-linked adrenoleukodystrophy (X-ALD) is characterized by two main phenotypes. Childhood cerebral ALD with onset of cerebral demyelination between 5-12 years results in a devastating neurologic degradation, and often death before adolescence. Adrenomyeloneuropathy (AMN) that affects adults males and heterozygous women is characterized by a pure myelopathy. However, about $35 \%$ of AMN males develop also cerebral demyelination with the same poor prognosis as in boys. The X-ALD ( $A B C D 1)$ gene codes for a peroxisomal transporter (ALD protein) are involved in the metabolism of verylong-chain fatty acids. Cerebral demyelination of X-ALD can be arrested in children and adults by allogeneic HSC transplantation (HCT) within 12-18 months, provided the procedure is performed at an early stage of the disease. The long-term beneficial effects of HCT in X-ALD are likely due to the progressive turn-over of brain microglia that are derived from myeloid progenitors in the bonemarrow. Despite the increased availability of cord blood, not all boys or adults with cerebral and who are candidate for HCT have a suitable HLA-matched donor. In addition, allogeneic HCT remains associated with significant mortality risk, particularly in adults. In two boys with cerebral ALD but without HLA-matched donor, we recently reported that HSC gene therapy with lentiviral vector has comparable effects to those seen after allogeneic HCT in arresting cerebral demyelination. Data on ALD protein expression as well as the identification of identical lentiviral insertion sites in myeloid and lymphoid lineages strongly suggested that HSCs were transduced in the patients. Hematopoiesis remained polyclonal without evidence of clonal skewing or dominance. Data will be presented on longer follow-up of these two treated patients, as well as on a third patient who has been treated more recently by HSC gene therapy.

\section{NEW GENETIC TECHNOLOGIES: PRACTICAL,} CLINICAL, AND SOCIETAL IMPLICATIONS

\section{S. Bale}

GeneDx, Gaithersburg, United States

Technologies developed in the research laboratory, especially those for analysis of many genes at a time, are being adapted to the clinical laboratory setting, and bringing with them issues related to sample preparation, turn-around time, sensitivity, data acquisition and interpretation, governmental regulation and oversight, and ethical complications.

\section{MENDELIAN GENETICS BY EXOME SEQUENCING}

M. Bamshad $1,2,3$

Pediatrics, University of Washington, United States

${ }^{2}$ Genome Sciences, University of Washington, United States

${ }^{3}$ Seattle Children's Hospital, Seattle, United States

Researchers, health-care providers and the public alike are interested in knowing to what extent a person's genetic makeup can help guide decisions about individual health and medical care. But finding genetic variants that influence human diseases has been a challenging task and remains one of the major obstacles to realizing the goal of personalized medicine. Over the past few years, great strides have been made toward developing new DNA sequencing technologies and computational tools for analysis of DNA sequence to help in the search for disease-causing variants throughout the human genome. To this end, it has recently become possible and affordable to sequence all the protein-coding exons of each gene in the human genome, otherwise known as the exome', in a single experiment. We have developed a strategy to use exome sequencing to find genes for monogenic disorders that have been intractable to conventional gene discovery. Our first application of this strategy identified the gene for a rare, autosomal recessive disorder of unknown cause, Miller syndrome (MIM \%263750). Specifically, comparison of the exomes of four affected individuals pinpointed a single candidate gene, $\mathrm{DHODH}$, which encodes a key enzyme in the pyrimidine de novo biosynthesis pathway. More recently, we used exome sequencing to identify the gene for a rare, autosomal dominant disorder, Kabuki syndrome (MIM \%147920). Specifically, nine of the ten affected individuals had mutations in a gene that encodes a Trithorax-group histone methyltransferase, a protein which is important in the epigenetic control of active chromatin states. These results demonstrate that exome sequencing of a small number of unrelated, affected individuals can be a powerful, efficient strategy for identifying the genes underlying rare Mendelian disorders that will likely transform the genetic analysis of monogenic traits. Subsequently, we are adapting exome sequencing to search for rare DNA sequence variants underlying the heritability of complex traits. This poses far more challenges. Use of exome sequencing for genetic analysis raises a number of important ethical questions: How many clinically relevant mutations reside in the exome of every individual? Should information about these mutations be given to each patient or research participant? How should decisions about the clinical relevance of mutations be made? And who should make these decisions? Developing a framework to study what are the best answers to these questions will be critical as exome, and eventually whole genome, sequencing becomes a standard instrument of scientists and clinicians. 
MECHANISMS OF NEURONAL DEATH IN BATTEN'S DISEASE

M. J. Bennett

Pathology \& Laboratory Medicine, The Children's Hospital of Philadelphia,

Philadelphia, United States

The neuronal ceroid-lipofuscinoses (NCLs) are a group of severe recessively inherited neurodegenerative disorders that are characterized by retinal pigmentary changes and vision loss, seizures, loss of motor and cognitive function and early death. The pathogenesis of neuronal loss has not been established. My laboratory has been defining the pathophysiology of Juvenile NCL (CLN3, Batten's disease). Many hypotheses have been suggested regarding the function of the CLN3 protein (Battenin) including neuronal anti-apoptosis via the ceramide pathway, synaptic neurotransmission, vacuolar $\mathrm{pH}$ regulation, an autoimmune reaction to neuronal proteins, and endosomal/lysosomal trafficking. We have shown Battenin to function in a novel palmitoylprotein delta-9 desaturase pathway. We have developed a siRNA knock down model in cultured neuroblastoma cells to study pathways that are perturbed in Battenin deficiency. This model was shown to have increased intracellular calcium which we hypothesized would be a trigger for neuronal apoptosis. We have studied the effects of a panel of 42 calcium channel modulators on this abnormal calcium content to determine if any of these drugs can modulate the effect and thus be candidates for therapeutic intervention. 7 were shown to normalize the abnormal calcium content and some of these drugs are available for different indications. Although the pathogenesis of CLN3 remains incomplete, it is now clear that at least part of the neuronal cell death is triggered by increased intracellular calcium content and the apoptosis pathway. This pathway can be modulated in vitro by the use of calcium channel antagonists that are already on the market for other indications and for which safety profiles are established. At the moment, it is not known if data from CLN3 studies can be applied to the other NCL's or other causes of neuronal death but if a common pathway is established then this may open up a significant therapeutic potential.

\section{MALDI-TOF CHARACTERISATION OF CDG DISORDERS}

F. Bowling ${ }^{1,2}$, T. M.Wallis ${ }^{2}$

${ }^{\prime}$ Biochemical Genetics, Mater Children's Hospital, Brisbane, Australia

${ }^{2}$ Mater Pathology, Brisbane, Australia

Introduction: The congenital disorders of protein glycosylation are a family of enzyme defects in assembly (Type I), transfer, and remodeling (Type II) of glycan structures to proteins. The features have wide variation and, while certain common presentations are known, the correlation between genotype and phenotype is not strong. Screening for these disorders in any unexplained syndrome is recommended. In protein glycosylation, the glycan structure is assembled in the endoplasmic reticulum and transferred for attachment to either an Asparagine residue with an $\mathrm{N}$-linkage or to Serine/Threonine residues with an O-linkage. The current method of screening for these disorders is IEF, which discriminates charge differences in Transferrin or Apolipoprotein C-III due to failure to attach sialic acid residues to an incomplete glycan. Charge based screens cannot differentiate individual enzyme defects, gives false positive results with protein variants, and reportedly cannot identify $11 / 44$ known disorders. We report the development and evaluation of MALDO-TOF/TOF methods for the characterisation of both $\mathrm{N}$ and O-glycan disorders. Methods: Briefly, the Apo C-III method uses acetonitrile for removal of albumin followed by TFA acidification and then MALDI ionisation on a DHB matrix. The $\mathrm{N}$-glycan method uses affinity chromatography to isolate IgG and Transferrin, a yeast enolase internal standard, sialidase and PNGase-F to release free glycans which are extracted on a carbon column, and then MALDI ionisation on a DHB matrix. Results: The Apo C-III method discriminated and quantitated all forms of the mucin Type $1 \mathrm{O}$-glycan. Comparison against patient samples identified COG7, ATP6VOA2, and two novel disorders of ApoC-III -1 and Apo C-III -2. The N-glycan method can identify and quantitate 15 glycan structures, and has confirmed primary abnormalities of PMM2, MPI, COG7 and also Galactosemia. Normal structures were apparent in protein variant samples with abnormal IEF patterns. Conclusion: MALDI-TOF mass analysis of glycans provides a more informative investigation of possible CDG patients.

\section{DETECTION OF CHROMOSOME MOSAICISM, CHIMERISM AND CLINICALLY SIGNIFICANT LONG CONTINUOUS STRETCHES OF HOMOZYGOSITY BY MOLECULAR KARYOTYPING WITH SNP MICROARRAYS}

\section{L. Bruno ${ }^{1,2}$}

${ }^{1}$ Cytogenetics, Victorian Clinical Genetics Services Pathology, Melbourne, Australia ${ }^{2}$ Murdoch Childrens Research Institute, Melbourne, Australia

Several studies have demonstrated the ability of SNP arrays, which provide genotyping data in addition to $\operatorname{LogR}$ 'copy number' data, for gene level autozygosity mapping, estimating low levels of mosaicism, assessing long continuous stretches of homozygosity (LCSH), uniparental disomy (UPD) and regions identical by descent. However, there remains little information on the clinical utility of SNP genotyping data for the investigation of individuals with intellectual disability, developmental delay, abnormal growth, autism or congenital abnormalities. In the course of testing 4000 clinical samples using the Illumina HumanCytoSNP-12 array our laboratory detected clinically significant 'LogR-neutral' genotyping abnormalities in approximately $0.7 \%$ of cases. Among these were a single case of chimerism, 1 segmental UPD, 11 cases involving low level chromosome mosaicism, 11 cases with a LCSH associated with a uniparental disomy, and a further 3 cases involving a LCSH harbouring a 'recessive type' genetic defect. These demonstrate the utility of SNP array analysis for detection of clinically significant ' $\operatorname{LogR}$ neutral' chromosome abnormalities, including chimerism/mosaicism down to 5-10\% and recessive Mendelian disorders associated with autozygosity. Notably, SNP genotyping data was available for 130 parental-proband trios, enabling estimation of the frequency of cryptic heterodisomy in this patient cohort. The growing clinical demand for SNP genotyping data poses significant challenges for the interpretation of LCSH, especially where there is no genotype-phenotype correlation. From an analytical perspective, genotyping patterns can be difficult to interpret and follow up testing using conventional cytogenetic and molecular methods is often required to delineate the genetic abnormality in detail. Finally, the ability of SNP arrays to reveal high degrees of consanguinity raises potential ethical and legal implications of reporting of such results.

\section{LABORATORY INVESTIGATION OF FATTY ACID OXIDATION DEFECTS}

\section{K. Carpenter, K. Sim}

Biochemical Genetics Service, Children's Hospital at Westmead, Westmead, Australia

The laboratory investigation of patients for Fatty Acid Oxidation Defects (FAOD) is often a multi-step process. Many FAOD can be detected on expanded newborn screening utilizing the accumulation of typical acylcarnitines species during the period of catabolism following birth. Confirmation of the defect may only require follow up plasma acylcarnitines and urinary organic acids with abnormalities being constantly present and virtually pathognomonic (e.g., MCADD). Some of the defects suspected on newborn screening however may have no urinary markers and may give normal plasma acylcarnitines when the child is well (VLCADD). As an alternative to stressing the patient we can use cultured fibroblasts and probe the $\beta$ oxidation pathway using stable isotope substrates and measure accumulating acylcarnitines species to pinpoint the defect or select the most appropriate direct enzyme assay to perform. Other FAOD do not result in acylcarnitine accumulation and will only be revealed by urinary organic acids during an acute catabolic episode (e.g., HMGCoA synthase deficiency). While mutation analysis is increasingly performed as a confirmatory test for suspected FAOD, interpretation of any sequence variation can be difficult. Analysis of biochemical markers in biological fluids has the advantage of offering a window into the metabolism of the patient and still has a role in diagnosis. 


\section{GENOME WIDE ASSOCIATION STUDIES - HAVE WE HAD ENOUGH ALREADY?}

\section{G. Chenevix-Trench}

Genetics, Queensland Institute of Medical Research, Brisbane, Australia

The explosion in genome wide association studies (GWAS) in the last five years has been remarkable, resulting in more than 800 published associations (at $p<5 \times 10^{-8}$ ) for more than 150 complex traits. The cynics profess disappointment at the proportion of risk for complex diseases that common SNPs currently appear to explain, but there is increasing evidence that there might be hundreds, or thousands, of common SNPs that contribute to these risks. Most of these SNPs will not be identifiable at these stringent significance levels, but cumulatively they will probably explain a large part of the variation in risk. With the advent of massively parallel sequencing, we can now expect an explosion in the identification of genes for rare Mendelian traits. However, neither of these approaches has yet been very successful at identifying genes for pharmacogenetic traits although there is considerable interest this area because of the potential for translation of this research into personalized medicine. Furthermore, there is a reasonable expectation that the effect sizes for pharmacogenetic traits might be quite large because of the lack of selective pressure, and so these genes might be identifiable with relatively small GWAS. The problem is that there is a paucity of studies available with both good quality germline DNA, and detailed clinical data from patients treated in a similar manner, which can be used for these analyses. We have carried out a GWAS for chemoresponse in ovarian cancer in the Australian Ovarian Cancer Study, and the publicly available The Cancer Genome Atlas, and will present the results of this study.

\section{GENETIC METABOLIC SEIZURE DISORDERS IN INFANCY}

\section{P. Clayton}

Clinical and Molecular Genetics Unit, UCL Institute of Child health, London,

United Kingdom

Faced with a neonate or young infant presenting with seizures, it is important to consider metabolic causes at the same time as considering meningitis, hypoxic ischemic encephalopathy etc. Examination may provide specific clues as may skeletal radiology, MR imaging and spectroscopy. A full work-up requires measurements of plasma sodium, calcium, magnesium, glucose, ammonia, lactate, LFT's (including alk phos), CK amino acids, biotinidase, urate, copper, transferrin isoelectric focussing, very long chain fatty acids, white cell CoQ and blood spot acylcarnitine profile. A paired CSF sample should be sent for analysis of glucose, amino acids, lactate, pyridoxal phosphate [PLP], neurotransmitter amine metabolites, 5 -methyl-tetrahydrofolate. Urine tests should include organic acids, sulphite, purine and pyrimidine metabolites, amino acids, aaminoadipic semiladehyde [AASA], creatine, creatinine and guanidinoacetic acid. A muscle biopsy may be indicated. For antiepileptic drug-resistant seizures, consideration should be given to trials of vitamin B6 (with resuscitation facilities available and with full EEG monitoring). Pyridoxine $50 \mathrm{mg}$ i/v daily for $3 \mathrm{~d}$, can be followed, if unsuccessful, by pyridoxal phosphate via $\mathrm{n} / \mathrm{g}$ tube $(50 \mathrm{mg}$ followed by $30 \mathrm{mg} / \mathrm{kg} / \mathrm{d}$ in 4 doses for $4 \mathrm{~d}$ ). Other specific treatments that may be indicated by test results include: biotin, folinic acid, serine \pm glycine, $\mathrm{CoQ}$, creatine, protein restriction, ammonia-lowering drugs, dialysis, copper histidinate, cyclic pyranopterin etc. DNA analyses to be considered include: Antiquitin (ALDH7A1) [raised urine AASA/response to pyridoxine], pyridox(am)ine phosphate oxidase (PNPO) [low CSF PLP/response to PLP], GLUT1 [CSF/plasma glucose ratio <0.4], POLG1 [raised lactate, progressive liver dysfunction], RARS2 [raised lactate, pontocerebellar hypoplasia, cerebellar cysts], SLC25A22 [MRI, reduced glutamate oxidation in fibroblasts], $C D K L 5$ [refractory tonic-clonic seizures, gross hypotonia, poor eye contact, later microcephaly and Rett-like features], $C T S D$ [slowing of head growth last trimester, spasticity, apnoea, granular osmophilic deposits in brain], FOLRI [progressive motor dysfunction, epilepsy and very low CSF folate].

\section{FROM PHENOTYPE TO MUTATION USING} MASSIVELY PARALLEL SEQUENCING

\section{M.A. Corbet}

Genetics and Molecular Pathology, SA Pathology, Adelaide, Australia

The intellectual disabilities (IDs) are a phenotypically heterogenous group of disorders with an estimated prevalence of between $1-3 \%$ of the population. Mutations associated with ID have been identified in over 200 genes that have diverse functional roles. Such diversity confounds novel mutation discovery using candidate gene screening approaches. We used sequence enrichment and massively parallel (or next-generation) sequencing technology to make a simultaneous and unbiased interrogation of all genes within disease associated chromosomal loci as identified by linkage. We present a summary of 11 different autosomal and entire X-chromosome exome projects using both chip and in-solution array based enrichment strategies capturing up to $5 \mathrm{Mb}$ of genomic DNA sequences. Between 4 and 8 million 65 base pair single end reads from the Illumina GAII platform were assembled to the hg18 human genome build. On average, 93\% of the bases in open reading frames (ORFs) that were also present on the array were covered with at least one sequence read. Based on data from the X-chromosome in males and autosomal regions homozygous by descent; 200-500 single nucleotide polymorphisms (SNPs) were identified for a single allele per Mbp of tiled sequence. We filtered out known SNPs comprising approximately $90 \%$ of the sequence variants we identified, using available public data and prioritized analysis of rare variants predicted to affect protein translation. Using this strategy we discovered and functionally validated (using mouse primary neuronal cells) a mutation in a previously uncharacterized, autosomal ARF6 GTPase binding protein, TBC1D24 in a family with focal epilepsy and intellectual disability. We also identified an 18 bp deletion in the OFD1 gene underlying X-linked Joubert syndrome. We conclude that sequence enrichment and massively parallel sequencing combined with appropriate functional validation is a highly effective strategy for mutation discovery.

\section{THE HUMAN VARIOME PROJECT -} PILOT PROJECTS AND PROGRESS

R. G. H. Cotton ${ }^{1,2}$

'Genomic Disorders Research Centre, Florey Neuroscience Institutes, Melbourne, Australia

${ }^{2}$ Department of Medicine, University of Melbourne, Australia

The Human Variome Project (Cotton et al. 2007) (www.human variomeproject.org) was initiated in June 2006 (Ring et al. 2006) drawing attention to the importance of collection of variation and its phenotype and to develop programs to put this into effect. The project builds on work and concepts of the HGVS over many years (www.hgvs.org) to focus on all variation associated with disease. The project will include those discovering mutations and their effects and then collecting the data making it instantly available for those who need it to inform clinical decisions, therapy, and research. A high level meeting in Spain (Kaput et al. 2009) developed plans to implement the recommendations of the HVP Melbourne meeting (Cotton et al. 2007). The HVP was featured recently in relation to Neurogenetic databases in Science (Cotton et al. 2008) and a HVP Neurogenetic Database Initiative is being formed after an HVP Neurogenetics forum in Hawaii in October 2009. The Nutrigenomics community is establishing protocols in partnership with HVP. This activity culminated in the third HVP meeting hosted by and under the patronage of UNESCO in Paris (www.humanvariomeproject.org). A manuscript on the Paris meeting is in preparation (Maija Kohonen Corish et al. 2010). The HVP and InSiGHT (International Society for Gastrointestinal Hereditary Tumours) (www.insight-group.org) has developed a major pilot study to develop procedures and systems to allow effortless flow of de-identified data for the colon cancer genes from the patient/clinic/diagnostic laboratory via curated locus or gene specific databases to central databases/genome browsers such as NCBI, UCSC and EBI. The system will be easily adaptable to other genes and to multiple laboratories, states and countries worldwide. A country specific collection pilot is underway in Australia and an 
International Confederation of these countries has been initiated with Malaysia, Korea, China, Australia and Saudi Arabia in the application process. Other pilot studies developed include specific ethical studies related to mutation collection, loading of LSDB content to NCBI databases, funding of curation of LSDBs, a system of Microattribution/reward for mutation submission.

\section{FROM PATERNALISM TO THE INTERNET:}

\section{ETHICS OF THE NEW GENETICS}

\author{
R. E. Duncan ${ }^{1,2,3,4}$ \\ ${ }^{\prime}$ Centre for Adolescent Health, Melbourne, Australia \\ Children's Bioethics Centre, Melbourne, Australia \\ Bruce Lefroy Centre for Genetic Health Research, Melbourne, Australia \\ Murdoch Childrens Research Institute, Melbourne, Australia
}

Until recently, the way genetic technology was presented to and used by the public was closely regulated by the medical profession and, some would say, rationed. Times have changed. Direct to consumer gene testing means that anyone with the interest plus a small amount of money can have a great slice of their personal genetic knowledge offered to them. As testing becomes faster and cheaper, financial constraints will cease to be a limiting factor. Because of the freedoms offered to international trade, there is no turning back from this scenario. What does this mean for the way in which we think about genetics, offer testing to individuals and support the public when they receive genetic information about themselves and their family? Drawing from a range of examples and theories, I will focus on the new ethical challenges we face in the field of genetic testing, and the changes these demand if we are to avoid being left behind.

\section{REPORTING INCIDENTAL FINDINGS IN WHOLE-GENOME}

\section{TESTING}

\section{W. Faucett}

Department of Human Genetics, Emory University School of Medicine, United States

The clinical use of whole-genome cytogenomic arrays provides a model system for learning how to communicate test results from diagnostic technologies such as whole-genome sequencing or exome sequencing. Cytogenomic arrays have enhanced the genetic evaluation of children with developmental disabilities. A recent consensus statement by (Miller et al., 2010) stated that cytogenomic arrays should be considered the first tier diagnostic test. In tested individuals the cause of their disability may be found but many pathogenic CNVs may also be found that are large, de novo or inherited events. These chromosomal changes may reveal unsuspected future health risks for these individuals and family members in addition to explaining the developmental disabilities. These 'incidental findings' include deletions of dominant genes for cancer (e.g., p53), cardiac arrhythmia (e.g., long QT syndrome), renal disease, diabetes and other serious conditions. How do we prepare patients, families and the clinical community for these unexpected clinically important results that are not related to the current clinical question? Knowledge of these specific risks may lead to life-saving interventions and preventive medical management. Developing methods to report and communicate these unexpected results and to ensure the information will be available at the appropriate age for intervention is a clinical challenge and will require the integration of this predictive health information into each patient's electronic health record. The International Standards for Cytogenomic Arrays (ISCA) Consortium is working with clinical genetic testing labs around the world and will be seeking consensus among member laboratories on how best to handle 'incidental findings'.

NUTRITION MANAGEMENT OF LONG-CHAIN FATTY ACID OXIDATION DISORDERS

M. Gillingham

Molecular \& Medical Genetics, Oregon Health \& Science University, United States

The primary goal of nutrition management in all long-chain fatty acid oxidation defects is to minimize fatty acid oxidation (FAO) by avoidance of fasting and providing adequate nonfat calories during stress, either orally or parenterally if needed. If dietary treatment is initiated immediately the acute symptoms such as hypoketotic hypoglycemia most often resolve and can be prevented. However, most patients develop some chronic complications such as recurrent rhabdomyolysis, or chorioretinopathy of LCHAD deficiency despite dietary treatment. New research on determining total energy needs, the role of dietary protein and the effects of MCT supplementation on exercise tolerance in patients with long-chain FAO disorders will be presented. Further advances in the nutritional management of long-chain FAO disorders will improve overall health and may decrease chronic complications associated with these disorders.

\section{INBORN ERRORS OF DOPAMINE METABOLISM, THEIR RECOGNITION AND MANAGEMENT}

\section{P. Grattan-Smith}

Neurology, Westmead Children's Hospital, Westmead, Australia

Inborn errors of dopamine are rare but important to recognize as they are treatable and inherited. Depending on the severity of the defect, onset of symptoms may be in the first months of life or later in childhood. It is common for the younger children to carry the mistaken diagnosis of 'cerebral palsy'. There are a number of clinical features that give clues to the presence of a dopamine deficiency disorder. In the infant these include oculogyric crises and a coarse tremor which may affect the limbs and the tongue. The infant may look 'myopathic' with a tented upper lip, ptosis drooling, and truncal hypotonia but arguing against a myopathy, limb tone is increased and the reflexes are very brisk. Bradykinesia can be extreme with child almost immobile. Older children may appear 'diplegic'. In school-age children with classic Segawa's syndrome, there is diurnal variation with the development of progressive leg dystonia over the course of the day. CSF neurotransmitters are important in confirming the presence of a dopamine deficiency state. They may also suggest the specific error, for example, an isolated reduction in homovanillic acid is typical of tyrosine hydroxylase deficiency. In older children with classic Segawa's disease, treatment with L-dopa is relatively easily. In younger children, especially if there is combined dopamine and serotonin deficiency, treatment may be very difficult. The presentation will include videos of representative cases.

\section{INTEGRATED TRANSCRIPTOME ANALYSIS} OF PANCREATIC CANCER

\section{S. Grimmond}

Queensland Centre for Medical Genomics, Institute for Molecular Bioscience, University of Queensland, Australia

Over the last decade, array-based transcriptome profiling has revolutionized our ability to survey gene activity and provided the foundations for many systems based analyses of tumorigenesis. It is now clear however that array expression profiling fails to adequately capture the true complexity and dynamics of the mammalian transcriptome and this has limited network analyses to gene centric approaches. As part of the International Cancer Genome Consortium, we have undertaken systematic sequencing of matched (tumour and normal) genomes, transcriptomes and methylomes in a large cohort of patients with the aim of defining the underlying molecular networks driving of pancreatic, ovarian and breast cancer. This exhaustive sequencing of the mRNA transcriptome has redefined our understanding of transcriptional output in individual patient samples by providing sensitive estimates of gene activity, promoter usage, alternative splicing and novel expression. Deep sequencing of the small RNA fraction from matched normal and tumour tissues have provided opportunities to model cancer related miRNA-mRNA networks playing roles in proliferation and cell cycle control. Most importantly, transcriptome sequencing has also provides the opportunity to also study variant expression in cancer, to assess whether somatic mutations found in the genome sequencing effort are active in the tumour. The impact of mutations on transcript-centric Vs genecentric models an also be addressed. Finally we will present how surveying interplay between genomic and transcriptome events can refine models of gene networks driving individual cancers. 


\section{A TRIP DOWN MEMORY LANE:} PROGRESS IN PRENATAL TESTING

J. L. Halliday ${ }^{1,2}$

${ }^{\prime}$ Public Health Genetics, Murdoch Childrens Research Institute, Melbourne, Australia

${ }^{2}$ Paediatrics, University of Melbourne, Australia

Prenatal diagnostic testing began in Victoria over 30 years ago and we were present right from beginning to report and monitor in detail the indications for testing, and the karyotypes and Mendelian disorders detected. This wealth of data has triggered numerous research projects, informed screening programs, played a role in policy development and education of women and health professionals. There have been many societal and technological changes that have influenced both the approach to and the methods associated with prenatal testing. The growth of consumerism in health care has produced many changes from a system where medically-driven decisions were the norm, to a time when shared decision making involves the individual and the health professions as partners; the goal of obtaining informed 'choice' for testing has replaced that of informed 'consent'. As an epidemiologist working alongside many clinical and laboratory-based geneticists, I have been able to witness and study aspects of many of these changes. This oration is an opportunity to reflect on 30 years of my research career to highlight some of the most interesting developments and outcomes related to prenatal testing, concluding with some future potential scenarios for consideration.

\section{CURRENT AND EMERGING THERAPIES} FOR LYSOSOMAL STORAGE DISORDERS

\section{J.Y. Lee}

Metabolic Medicine, Genetic Health Services Victoria, Murdoch Children's Research Institute, Melbourne, Australia

Lysosomal Storage Disorders (LSD) are a group of $>40$ disorders, the majority of which are due to deficient enzyme activity. Clinical aspects are characterized by considerable genetic and phenotypic heterogeneity. LSD are progressive conditions affecting multiple organ systems. Before the 1980 s, supportive treatment was the mainstay of therapy. However, in the last three decades, therapeutic options, although not curative, have been available. Bone marrow transplantation (BMT) has been used for certain LSD since the early 1980s. Transplantation can correct some clinical manifestations but it does not prevent disease progression of other. Additional limitations include the need to perform the BMT prior to onset of neurological symptoms, difficulty in finding matched donors and graft failure. Enzyme Replacement therapy (ERT) is currently commercially available for Gaucher, Fabry, and Pompe diseases, and for Mucopolysaccharidoses I, II and VI. ERT has been effective in improving many symptoms of these LSD without significant side effects. However, a major limitation is the inability of the enzyme to cross the blood brain barrier, hence, not affecting the CNS. ERT is also very expensive and requires frequent hospital visits disrupting school and family life, adding to the disease burden, which is extremely high in any case. Substrate Reduction Therapy (SRT) involves small molecules acting on the synthetic pathways to reduce substrate production. N-butyldeoxynojirimycin (Miglustat, Zavesca) is currently an accepted alternative to ERT for non-neuropathic Gaucher. It has also been shown to have some benefits in GM2 gangliosidoses and Niemann-Pick Type C. However, its efficacy depends on the presence of residual enzyme activity. Research is ongoing for other modalities such as gene therapy, CNS directed therapy, chaperone therapy, stop codon read through and use of genistein. These options might serve as future therapies or as adjunct to the existing ones, overcoming their limitations.

\section{PATIENT-DERIVED NEURAL STEM CELLS FROM PATIENTS:} NOVEL MODELS FOR BRAIN DISEASES

A. Mackay-Sim

National Centre for Adult Stem Cell Research, Griffith University, Australia

Understanding complex genetic and environmental contributions to brain diseases would benefit from relevant and robust patient-derived cell models which carry the full complement of genetic variation and not simply an identified mutation in a gene of interest. Patientderived fibroblasts and lymphocytes are commonly used but may not reflect nervous system biology. Olfactory mucosa, the organ of smell, is a regenerating part of the nervous system in which neurogenesis continues throughout life making new sensory neurons from a multipotent neural stem cell. Our novel cellular model is based on cells from olfactory mucosa biopsies obtained from healthy controls and patients. Biopsies are grown as neurospheres in defined medium before dissociation and growth as adherent monolayers. Our initial experiments investigated whether olfactory neurosphere-derived cell lines from patients would reflect biologies relevant to schizophrenia, a neurodevelopmental psychiatric disorder, or sporadic Parkinson's disease, a neurodegenerative disease. By comparing 42 patient and control cell lines we demonstrated significant disease-specific alterations in gene expression and protein expression, including dysregulated neurodevelopmental pathways in schizophrenia and dysregulated mitochondrial function, oxidative stress and xenobiotic metabolism in Parkinson's disease. Subsequent cell functional assays confirmed alterations in specific neurodevelopmental functions in schizophrenia and xenobiotic metabolism and responses to environmental toxins in Parkinson's disease. Given the difficulty in identifying causal genes for many diseases, it is notable that significant disease-specific alterations in gene expression and cell functions can be identified in relatively small groups ( 8 patients and 8 controls) and replicated in independent groups, even in genetically complex diseases like schizophrenia and Parkinson's disease. It is possible that genetic (and epigenetic) factors get amplified in the dynamic homeostasis of an appropriate cellular model. This is illustrated in Hereditary Spastic Paraplegia in which a single gene mutation causes altered transcription of $60 \%$ of expressed genes with little change in cell function.

\section{IDENTIFICATION OF CNVS USING ARRAY CGH THE INTERPRETATION DILEMMAS OF THIS NEW TECHNOLOGY}

J. Nicholl', W.Waters', E. Haan², E. Thompson², C. Barnett' ${ }^{2}$, S. Yu

Cytogenetics, SA Pathogoly - WCH site, Adelaide, Australia

${ }^{2}$ SA Clinical Genetics, SA Pathology, Adelaide, Australia

Microarray technology is now widely used to diagnose pathogenic copy number variants (CNVs) in neurological disorders (Intellectual disability, autism, schizophrenia). As powerful as microarray technology is, interpretation of CNVs can be difficult and invariably requires follow up in the patient or other family members. Microarray also has the potential to unmask CNVs which are unrelated to the patients neurological presentation, but have long term deliterious consequences. To illustrate this point, three case studies will be discussed in detail. Case 1: A $2.66 \mathrm{Mb}$ interstitial deletion of 21q22.11-q22.12 was detected in a child with multiple malformations and developmental delay. The deletion resulted from an unbalanced insertional translocation between chromosomes 21q and $7 \mathrm{p}$ and included the gene RUNX1. Deletion of this gene predisposes the patient to development of myeloid malignancies. Many difficult counseling issues needed to be raised with the parents that went beyond finding a cause of the developmental delay and malformations in their child. Case 2: This invoved a sib-pair; both with an interstitial deletion of the region 16q11.2-q21.1. Examination of relevant databases indicated that this appeared to be a rare $\mathrm{CNV}$. Family studies showed co-segregation of the 16q deletion with learning difficulties. Is this then enough evidence to suggest that this change is responsible for the intellectual disability in this family? Case 3: This study involved a family with six children, all diagnosed with autism spectrum disorder. The etiology of autism spectrum disorder is complex with multiple candidate genes and suscepibility loci or genes reported. Array CGH did not show a single CNV affecting all family members, differing CNVs were seen in two or more family members; these included gain of $2 \mathrm{p} 21.1$, loss of $5 \mathrm{q} 12.3$ and gain of $16 \mathrm{q} 11.2$. This family presented a challenge when determining the significance of these copy number changes. 


\section{DATABASES AND GENETIC VARIATION}

S. J. Patton

Genetic Medicine, Central Manchester University Hospitals NHS Foundation Trust, Manchester, United Kingdom

In this talk I discuss the collection, interpretation, reporting and storage of gene variant data. The documentation and analysis of genetic variation has been a major theme of genetics, arising from research into the causes of disease and phenotypic traits and routine clinical genetic analysis. Interpretation of the effect of discovered variants continues to be a major challenge. Collection of variant data in databases has been a common response to this challenge: these databases range in scope and aim from core databases for all locuses to locus-specific databases covering single genes or diseases in great depth, some publicly available, some limited to defined groups; and from collections of large-scale structural variants to single base changes. In addition, the source of the variant data varies from the results of research, through re-sequencing projects to the outcomes of medical diagnoses, and this affects the standard of the data in terms of both the quality assurance applied during analysis and at the time of reporting and the depth and nature of the data sets generated. With reference to the Diagnostic Mutation Database project in the UK, I discuss how diagnostic laboratories have approached the problem of collecting and sharing variant data in order to aid interpretation, and how this has related to other databases and projects worldwide. Particular issues that we will discuss include data protection and governance, the challenges of data collection, security, standardisation, modeling, exchange, sustainability and federation of resources.

\section{THE DOSAGE-SENSITIVE AND -INSENSITIVE GENETIC LOCI: CAN WE DISCOVER WHICH IS WHICH? AND SO PREDICT THE CRITICAL CNVS?}

G. B. Peters, A. P. Darmanian

Cytogenetics, The Childrens Hospital at Westmead, Westmead, Australia

DNA microarray testing for constitutional change in gene copy number has become common in clinical genetics laboratories. This technology has improved detection of clinically relevant copy number changes, from around 3-4\% [as found via conventional cytogenetics], to $12 \%-15 \%$ of relevant test referrals. It has also made possible the discovery of new genomic disorders [eg the 17q21.31 and 2q23.1 microdeletion syndromes]. A major problem, though, has been the simultaneous detection of many copy number variants $[\mathrm{CNV}]$ of unknown clinical significance. These latter require exclusion as potential causes of disease, usually by expensive and laborious follow-up testing. Can we avoid some of this follow-up in the near future, or must we wait till the human genome, and its expression, is 'fully understood'? An a priori ability to discriminate benign from pathogenic CNVs would be desirable, especially when family testing proves inconclusive, or is unavailable. But is such prediction possible? Two recent studies have made a start on this problem. One study has produced a 'naïve Bayesian tree classifier' named 'GeCCO' [for 'Genomic Classification of CNVs Objectively'], while a second is now an adjunct to the 'Decipher' consortium database. The latter aims to estimate what genes [of 17,000 annotated so far] are the more dosage-sensitive, via a 'haploinsufficiency score'. If they achieve sufficient power, these new discriminating algorithms may teach us much about what sorts of genes are dosage-sensitive, and so aid in prediction of clinical impacts. We apply both discriminators to some of our own set of 2000 array test cases, to evaluate the utility of these approaches.

\section{FINALLY DIAGNOSED — SO WHERE TO FROM HERE?}

\section{Petrie}

Association of Genetic Support of Australasia, NSW, Australia

The Association of Genetic Support of Australasia (AGSA) was established in 1988 as an umbrella group for genetic conditions so rare that there is no support group. AGSA provides peer support, information and contact with others with the same or similar condition. AGSA has an extensive Database/Contact Register representing over 850 genetic conditions in 2200 families. AGSA is sandwiched between science and affected families. With the development of micro array testing, AGSA experienced an increase in enquiries regarding chromosomal abnormalities. There is also a significant increase in callers requesting information on who is researching their particular genetic condition, how to get up-to-date research information, who is the clinical expert on their condition, and what are the treatment options. Unfortunately science is ahead of the support capabilities. Communication and collaboration with support groups is lacking. In Australia, there are poorly coordinated services. Those affected by rare conditions are part of a fragmented system lacking any holistic approach. In Europe, Eurordis established a rare disease task force and was successful in changing European legislation to protect the rights of 30 million people with rare diseases and lobby for clinical centres of expertise throughout Europe. In Australia, the AGSA is in a strong position to work with other parties to achieve similar outcomes for the 1.5 million people with rare diseases, of which 300,000 are children. AGSA is currently collaborating with the Australian Paediatric Surveillance Unit, Steve Waugh Foundation and SMILE to research the impact of rare diseases in Australia. This presentation will outline what various support groups have achieved in the area of research and how AGSA can be the interface between researchers and families to provide first hand information on what individuals and families need for future successful collaboration. It is our genome, our mutation and it should be our future.

\section{GENE MUTATION AND PATIENT REGISTRY DATABASES -} TRANSLATING THE IDEAL INTO PRACTICE

D. Ravine ${ }^{1,2}$

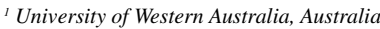

${ }^{2}$ PathWest, WA, Australia

Many internet-accessible relational databases with details of genetic variants within disease-associated genes are now available. The initial drive for creating these databases arose from research scientists, many of whom were involved with gene discovery. The early beneficiaries of 'locus-specific' databases were genetic diagnostic laboratories, which required ready access to information that allowed variants to be categorized from the viewpoint of disease causality. Although many hundreds of mutation databases are now available, the degree to which they achieve their aim of providing a comprehensive picture of the range and frequency of gene variants with the gene(s) of interest, as well as an accurate summary of the phenotypic consequences of each variant often falls short of the ideal. This has remained an ongoing significant issue that hinders those who are required to evaluate the likely clinical significance of rare gene variants, as well as hindering opportunities for future research. More recently, internet-accessible rare genetic disease-specific registries of patients are being established, many driven by the prospect of therapeutic trails. However the information stored within registry databases about genetic variants is typically restricted to details about the primary disease-causing mutation. There is a need now to upgrade the process of amassing details of all identified variants within genes associated with disease phenotypes and, at the same time, ensure that these details are integrated with the high-quality clinical details now being gathered within genetic patient registries.

\section{OVERVIEW OF GENE THERAPY FOR PROSTATE CANCER}

\section{P.J. Russell}

Australian Prostate Cancer Research Centre - Queensland, Institute of Health and Biomedical Innovation, Queensland University of Technology, Australia

Thirty years from its initiation, human gene therapy is now in an exciting phase, due to clearer principles of biology, progress in genomics and proteomics and developments in genetic engineering. Strategies for cancer gene therapy require an understanding of the biology of that cancer, and include targeting a defective gene, such a tumour suppressor gene, upregulation of drug resistance genes on 
normal tissues plus chemotherapy, down-regulation of specific genes using siRNA, targeting tumour angiogenesis, targeted killing within a non-essential organ, or upregulation of anti-cancer immune responses. Late stage castrate resistant prostate cancer (CRPC) remains incurable. Novel alternatives such as transcriptionally or transductionally targeted cytoreductive Gene Directed Enzyme Prodrug Therapy (GDEPT) offer great hope, the prostate being dispensable. They generate GDEPT-associated bystander effects - in situ amplification of cytotoxicity (local bystander effect) and potentially an immune response to eliminate micrometastases (distant bystander effect). Our team has used an ovine atadenovirus with transcriptional targeting of GDEPT to generate a new prostate targeted gene medicine effective against hormone-sensitive and CRPC xenografts and in TRAMP mice; this treatment will progress to Phase I clinical trial. Despite progress, even targeted gene therapy suffers from inefficient gene transfer in the clinical scenario. Combining strategies offers improved results and can overcome prostate cancer heterogeneity, allowing lower dosing to be used, with improvement in the quality of life. Combining GDEPT with immunotherapy (interleukin-12 plus interleukin-18) in an immunocompetent mouse model led to increased growth reduction of local prostate cancer, reduced pseudometastases to the lungs and provided a clear survival advantage compared with either treatment alone. Synergy between GDEPT and docetaxel treatment (the preferred option for CRPC) has also been achieved in immunocompetent mouse models. Other novel approaches include combining viral/nonviral gene vectors with nanotechnology (based on preferential accumulation of nanoparticles within tumour cells), allowing concomitant therapeutic delivery and real time imaging. These methods provide great potential for translation to the clinic for men with late stage prostate cancer.

\section{CNV IN THE EPILEPSIES}

\section{E. Scheffer}

Department of Medicine, Austin Health, University of Melbourne, Australia

The key role that copy number variants (CNV) play in causation of disease has become increasingly apparent in recent years. The importance of CNV in the epilepsies is only just emerging and providing a complex and intriguing picture of their role in this group of serious neurological disorders. As in intellectual disability, CNV may be the monogenic cause of severe epilepsies often in the setting of dysmorphic features and other congenital anomalies such as malformations of cortical development. Of greater novelty are the increasing reports of $\mathrm{CNV}$ underlying severe epilepsy syndromes that may occur without other stigmata to suggest a genetic etiology. Here a CNV in a single patient with a specific syndrome may point to a specific gene that can then be interrogated for mutations in other patients with the same epilepsy syndrome. Following our discovery of PCDH19 in the rare familial epilepsy syndrome of Epilepsy limited to Females with Mental Retardation (EFMR), another group found a CNV including just PCDH19 in a boy with Dravet syndrome. They then found 13 girls with Dravet syndrome who had mutations of PCDH19 highlighting this gene as the 'second' gene for Dravet syndrome although subtle phenotypic differences exist. Larger studies of CNV in epileptic encephalopathies are yielding interesting findings in terms of monogenic causes. Most common forms of epilepsy are not associated with intellectual disability and follow complex rather than monogenic inheritance. It was therefore surprizing when the well-described $15 \mathrm{q} 13.3$ microdeletion was found in $1 \%$ of the commonest group of genetic epilepsies, the Genetic Generalized Epilepsies (GGE, formerly called Idiopathic Generalized Epilepsies) compared with schizophrenia $(0.2 \%)$, autism $(0.4 \%)$, epilepsy with intellectual disability $(0.3 \%)$ and $0.015 \%$ of controls. Familial segregation of the 15q13.3 microdeletion shows the pattern expected of a susceptibility variant; it does not track with affected status and may be non-penetrant. The odds ratio of having epilepsy for a person carrying the $15 \mathrm{q} 13.3$ microdeletion is 68 increasing their risk of GGE from 1 in 80 to 1 in 3 . Several microdeletions have now been identified in GGE and while 15q13.3 remains the most frequent, $2 \%$ of GGE patients have a specific microdeletion likely to contribute to the etiology of their epilepsy.

\section{MICROARRAYS IN THE CLINICAL LABORATORY: DIAGNOSING CHROMOSOME ABNORMALITIES AND SINGLE GENE DISORDERS}

\section{G. Shaffer}

Signature Genomic Laboratories, Spokane, WA, United States

Microarrays have been used for clinical diagnosis since $\sim 2004$. The collective experience shows that microarrays have advantages over conventional karyotyping including higher resolution, comprehensive whole genome coverage, and detection rate of $15-20 \%$ for abnormalities. Based on conventional cytogenetics, there have been some unexpected results, such as higher rates of mosaicism and for certain rearrangements (i.e., insertions) based on what could be visualized through the light microscope. In addition, through a 'genotype-first' approach, microarrays have allowed for identification of previously unrecognized genetic disorders and elucidated the etiologies of known syndromes. We have studied over 43,000 cases submitted for array testing for a variety of clinical indications. Over 10,000 abnormalities have been identified. Although most are large structural changes in the genome, more than 400 of the abnormalities are intragenic alterations, most of which are deletions of single or multiple exons within a gene. As the resolutions increase, copy number changes are frequent and pose challenges to the clinical interpretation of the data. While much of karyotyping can be replaced by microarray testing, FISH is an indispensible adjunct to microarray analysis. Microarrays will identify changes in DNA copy number, but they cannot reveal the specific rearrangements responsible for these changes or the mechanisms of their formation. Therefore, the information is incomplete and counseling is not accurate in the absence of a clear understanding of these mechanisms.

\section{WHAT IT TAKES: INTRODUCING GENETIC TESTS INTO THE AUSTRALIAN HEALTH CARE SYSTEM}

\section{G. Suthers}

SA Clinical Genetics Service, SA Pathology, North Adelaide, SA, Australia

In the 1980s, genetic testing in Australia was provided by enthusiasts operating from research laboratories. Diagnostic genetic testing was, in effect, a cottage industry. With dramatic increases in the scope, demand, and utility of genetic tests, the discipline has had to adapt to the mainstream regulatory and funding mechanisms that apply to other forms of medical testing. In 2010, a diagnostic genetic test provided in Australia must be safe to administer, having approval from the Therapeutic Goods Administration. The test must be funded by the Federal (Medicare) or State governments, or be paid for by the patient. At present, there is a marked disparity in the rates of genetic testing in different jurisdictions, and a Federal mechanism will probably be essential to ensure equity of access to genetic tests. Federal funding can be in the form of additional funding for Medicare or modification of current items on the Medical Benefits Schedule; there are assessment hurdles associated with each pathway. The test must be performed by an accredited laboratory under the supervision of an accredited laboratory professional (medical scientist or pathologist). There are different regulatory agencies covering the laboratories and personnel requirements. The standards for accreditation provide a mandatory framework for the delivery of genetic testing. The utilisation of genetic tests rests with health professionals who do not necessarily have accredited training in medical genetics. At present, there is no mandatory regulatory mechanism for managing the utilisation of genetic tests in clinical practice. This implementation process can be challenging for a simple genetic test. It is a daunting challenge to consider the implementation of new forms of genetic testing (such as whole genome sequencing) or new contexts for testing (such as direct-to-consumer testing). 


\section{Submitted Abstracts}

\author{
LIVER TRANSPLANT FOR MAPLE SYRUP URINE DISEASE - \\ A FIRST FOR NEW ZEALAND \\ R. M.Akroyd, S. Chin, C.J.Wilson \\ Starship Children's Health, Auckland, New Zealand
}

Classical maple syrup urine disease (MSUD) is a metabolic disorder caused by a branched-chain $\alpha$-keto acid dehydrogenase complex deficiency. Early diagnosis and treatment is essential to prevent a metabolic crisis and neurological insult. Treatment includes a very low leucine diet, supplemented with a BCAA free formula, maintaining plasma levels of BCAAs in the mildly elevated range; and inhibition of muscle breakdown in times of catabolic stress. Recently, liver transplantation in MSUD has been shown to normalise BCAAs and enable an unrestricted protein intake. The proband, a 4-year-old female, presented day 7 of age with a 48-hour history of lethargy and poor feeding. At time of presentation the newborn screening results taken on day three of age showed a 'leucine' of 1536 $\mu \mathrm{mol} / \mathrm{L}(N=59$ $187 \mu \mathrm{mol} / \mathrm{L})$ with a significant amount of alloisoleucine. A repeat leucine was $2878 \mu \mathrm{mol} / \mathrm{L}$, confirming MSUD. She required hemofiltration and by day three of admission, leucine was $232 \mu \mathrm{mol} / \mathrm{L}$. She was discharged day 14 (age 21 days) managing full oral feeds. Since diagnosis and initial stabilisation, she has maintained exceptional metabolic control, with a mean leucine $206 \mu \mathrm{mol} / \mathrm{L}$. She has normal intelligence and no evidence of cognitive insult from the neonatal presentation. The parents requested a liver transplant. International consultation was sought and extensive discussion of diet treatment versus liver transplant for MSUD undertaken. She was listed on the donor waiting list aged 3.8 years. At 4.3 years she received a deceased donor liver; post transplant leucine at 12 hours was $188 \mu \mathrm{mol} / \mathrm{L}$ and subsequent levels have remained normal. She has had two early rejection episodes post transplant and at fourteen months post transplant remains well. This case demonstrates liver transplant is an option for MSUD. Liver transplant is not without risks and parents need to be fully informed and counseled on the possible morbidities and complications of transplantation.

\section{NOVEL INSIGHTS INTO THE ETIOLOGY OF 11 P15 IMPRINTING DISORDERS FROM A COHORT ANALYSIS OF BECKWITH WIEDEMANN AND RUSSELL SILVER SYNDROMES}

E. M. Algar,V. Dagar, M. Sebalj

Molecular Oncology Laboratory, Murdoch Children's Research Institute, Melbourne, Australia

For ten years our laboratory has provided genetic testing for Beckwith Wiedemann syndrome (BWS), and more latterly for Russell Silver syndrome (RSS), growth disorders that are commonly characterized by opposing imprinting defects, affecting Imprinting Centre 1 (IC1) within the H19 locus on $11 \mathrm{p} 15.5$, and by perturbations to a proximal 11p15 imprinting centre (IC2) involving KCNQ1OT1/LIT1, in BWS alone. Analysis of the mutation spectrum in BWS and RSS has led us to develop new hypotheses concerning the etiology of abnormal imprinting. Of 149 BWS cases positive by molecular testing, $28(19 \%)$ had mosaic paternal UPD $11 \mathrm{p} 15,12(8 \%)$ had isolated gain of methylation (GOM) at IC1, 94 $(62 \%)$ had isolated loss of methylation (LOM) at IC2, one had 11 p15 trisomy (pat dup) and three (2\%) CDKN1C mutations. Four families were identified with $11 \mathrm{p} 15$ copy number variations in 11 individuals. 133 cases with clinical features of RSS were tested. 28/133 (21\%) had LOM at IC1 and one case, a deletion within IC1 $(1 / 29,3.4 \%)$. Gender differences were noted in BWS and RSS mutation subgroups. In BWS, females were more commonly affected by 11p UPD (17F: 11M), and by IC2 LOM (53F: 41M) and were more likely to have complete versus partial IC2 LOM $(P=.084)$, suggesting that XX influences IC2 methylation in early embryogenesis. In contrast, in RSS, IC1 defects were more common in males (19M : 9F) $(P=.093)$, suggesting that XX protects against IC1 LOM. Reproductive history was available for $28 \mathrm{BWS}$ cases with IC2 LOM and UPD. Nine cases with IC2 LOM (9/20, 45\%) were conceived using ART. Of eight cases with 11p UPD, four mothers had a history of recurrent miscarriage (3) or gonadotrophin treatment (1). These findings suggest that infertility or infertility treatments are directly implicated in BWS and deserve further investigation.

\section{EVALUATION OF THE USE OF DRIED BLOOD-SPOT HOMOCYSTEINE MEASUREMENT FOR NEONATAL DETECTION OF HOMOCYSTINURIAS \\ K. Carpenter ${ }^{1,2}$, A. Alodaib $^{2}$,V.Wiley ${ }^{2,3}$, T.Wooton $^{3}$, B.Wilcken ${ }^{1,2,3}$ \\ ${ }^{1}$ Biochemical Genetics Service, Children's Hospital at Westmead, Australia ${ }^{2}$ Discipline of Paediatrics and Child Health, University of Sydney, Australia ${ }^{3}$ NSW Newborn Screening Programme, Children's Hospital at Westmead, Australia}

Newborn screening for the homocystinurias by tandem mass-spectrometry involves initial measurement of methionine to detect cystathionine $\beta$-synthase (CBS) deficiency, and propionyl and acetyl carnitines $(\mathrm{C} 3, \mathrm{C} 2)$ to detect cobalamin C (CblC) defect. Sensitivity is poor for CBS deficiency and specificity somewhat poor for both. Measurement of total homocysteine in dried blood spots has been used as a second-tier test in screening for CBS deficiency, and recently as an initial test. We have measured homocysteine in dried blood spots in normal newborns, and retrospectively in newborns with established disorders, using an LC-MS/MS method. The reference range for current neonatal samples was 5.4-10.7 $\mu \mathrm{mol} / \mathrm{L}$, and for samples stored for 10 years was $1.7-5.5 \mu \mathrm{mol} / \mathrm{L}(n=50)$, markedly reduced. The neonatal dried-blood spot homocysteine, measured retrospectively in five patients with confirmed CBS deficiency, was 21.6 to $43.6 \mu \mathrm{mol} / \mathrm{L}$, and that in four $\mathrm{CblC}$ deficient patients was $40.7,58.5,60.6,68.5 \mu \mathrm{mol} / \mathrm{L}$. Ages at sampling were 2-4 days, and sample ages at testing 1-12 years. All CBS patients were pyridoxine non-responsive, and initially detected by raised methionine levels at routine newborn screening. No newborns with pyridoxine-reponsive CBS deficiency have been detected. The cobalamin $\mathrm{C}$ deficient patients were detected because of elevated $\mathrm{C} 3 / \mathrm{C} 2$ ratios, without elevated $\mathrm{C} 3$ alone. We conclude that pyridoxine-nonresponsive CBS deficient patients have very clear elevations of homocysteine at 2-4 days of age. The sensitivity of this test for pyridoxine-responsive patients remains unknown. The newborn levels found in cobalamin $\mathrm{C}$ deficient babies were even higher, and seem fully discriminatory.

\section{EXPERIENCE WITH A UPLC-MS/MS METHOD FOR ANALYSIS OF BRANCHED CHAIN AMINO ACIDS IN DRIED BLOOD SPOTS \\ A. Alodaib $^{3}$, K. Carpenter ${ }^{1,3}$, V.Wiley ${ }^{2,3}$, K. Sim ', B.Wilcken ${ }^{1,2,3}$ \\ ${ }^{\prime}$ Biochemical Genetics Service, Children's Hospital at Westmead, Australia ${ }^{2}$ NSW Newborn Screening Programme, Children's Hospital at Westmead, Australia ${ }^{3}$ Discipline of Paediatrics and Child Health, University of Sydney, NSW, Australia}

Analysis of branched chain amino acids is required for diagnosis and management of patients with Maple Syrup urine Disease. Tandem mass spectrometry (MS/MS) based newborn screening is unable to distinguish between isobaric leucine species so identification of the pathognomonic marker, alloisoleucine, requires further testing. Traditional ion exchange chromatography is time consuming and plasma assays require venipuncture and transport of samples to the reference laboratory. Second tier testing of the initial dried blood 
spot (DBS) combining liquid chromatography with MS/MS has been used to improve specificity for MSUD newborn screening. We report our experience in using such a method and highlight the utility of the method for monitoring patients from home using dried blood spots and as a backup for traditional plasma assays. The method is based on that of Oglesbee et al. and utilises stable isotope labeled internal standards for valine, alloisoleucine and leucine. Analytes are separated using ultra high performance liquid chromatography with gradient elution with a total run time of only 5 minutes. DBS standard values were assigned to give optimal correlation for DBS results with plasma samples taken at the same time and analyzed by amino acid analyser (Biochrom 30). Comparison of 60 plasma values with DBS taken at the same time showed excellent correlation for all analytes, $\left(r^{2}>0.97\right)$. Within batch precision ranged from $3 \%$ for Leucine to $10.67 \%$ for Isoleucine, between batch precision is typically around $10 \%$.

\section{CAN THE MULTIDIMENSIONAL MEASURE OF INFORMED CHOICE (MMIC) MEASURE INFORMED DECISION MAKING IN POPULATION CARRIER SCREENING FOR FRAGILE $X$ SYNDROME?}

A.Ames ${ }^{1,2}$,A.Archibald ${ }^{1,2}$, M. Hill ${ }^{1,7}$, M. Martyn', O. Ukoumunne ${ }^{3}$, J. Cohen ${ }^{5,6}$, J. Emery ${ }^{4}$, S. Metcalfe ${ }^{1,2}$

${ }^{I}$ Genetic Education and Health Research, Murdoch Children's Research Institute, Melbourne, Australia

${ }^{2}$ Department of Paediatrics, University of Melbourne, Melbourne, Australia ${ }^{3}$ Clinical Epidemiology and Biostatistics, Murdoch Childrens Research Institute, Parkville, VIC, Australia

${ }^{4}$ Department of General Practice, The University of Western Australia, Australia ${ }^{5}$ Fragile X Alliance, Melbourne, Australia

${ }^{6}$ Centre for Developmental Disability Health Victoria, Department of General Pract, Monash University, Australia

${ }^{7}$ Clinical and Molecular Genetics, Institute of Child Health and Great Ormond Street Hospital for Children, NHS Tru, London, United Kingdom

Informed decision-making (IDM) is important so that individuals can make autonomous decisions without coercion or deception. Models have been developed to measure IDM in prenatal Down syndrome screening, eg the multidimensional measure of informed choice (MMIC), plus a deliberation (D) scale to measure weighing up of the pros and cons (i.e., IDM = MMIC+D). These models have not been applied to carrier screening for inherited conditions. We are currently offering carrier screening for fragile X syndrome (FXS) to non-pregnant women from the general population. Existing models were applied to examine IDM in this context. 156 women completed a questionnaire when deciding about testing. The questionnaire contained scales of FXS knowledge, attitudes and deliberation, as well as other questions. The MMIC and IDM models were applied to these data. Results of these measures were: test uptake (85\%); good knowledge $(78 \%)$; positive attitudes $(79 \%)$; deliberated $(86 \%) .71 \%$ of women made an 'informed choice' (i.e., tested, having good knowledge and positive attitudes or not tested, good knowledge and negative attitudes). 64\% of women made an 'informed decision' (an informed choice and deliberated). A subgroup of 28 women were interviewed. Interview responses were coded by three independent researchers using content analysis. Responses from these semi-structured interviews were compared with responses in the questionnaires to triangulate the findings. Interview and questionnaire findings did not concur in 12/28 cases, that is, 12 women were misclassified according to the measures. Factors other than poor knowledge, primarily the inconsistency between attitudes and behavior contributed to an apparent lack of IDM. These factors, such as not planning a family at the time or apparent lack of deliberation because of prior experiences, are not captured by these models. To assess IDM in population carrier screening, alternate approaches to the MMIC/MMIC+D models may be needed to account for these other factors.

\section{THE MERRY-GO-ROUND OF DISCOVERY: FAMILIES' EXPERIENCES OF THE DIAGNOSIS OF FRAGILE X SYNDROME}

A. D. Archibald ${ }^{1,2}$, A. M. Jaques ${ }^{2,5}$, S. Wake ${ }^{2,3}$, J. Cohen ${ }^{4}$, S. A. Metcalfe ${ }^{1,2}$

${ }^{\prime}$ Genetics Education and Health Research, Murdoch Childrens Research Institute, Melbourne, Australia

${ }^{2}$ Department of Paediatrics, The University of Melbourne, Australia

${ }_{3}^{3}$ Genetic Health Services Victoria, Melbourne, Australia

${ }^{4}$ Fragile X Alliance Inc., Melbourne, Australia

${ }^{5}$ Public Health Genetics, Murdoch Childrens Research Institute, Melbourne, Australia

Fragile X syndrome (FXS) is the leading cause of inherited intellectual disability. Research indicates that there are often long delays between the time parents first notice their child is not meeting developmental milestones and when a child is diagnosed with FXS. We explored the experiences of relatives of people with FXS as part of a larger study investigating screening for FXS. Here we report how relatives experienced, coped and adapted to the diagnosis of FXS. A qualitative approach was taken involving in-depth interviews with 29 relatives of people with FXS in Victoria, Australia. Relatives included parents, grandparents, aunts, uncles, siblings and cousins. The data were analyzed using thematic analysis. Participants gave in-depth insights into their experiences of having a child or relative with FXS and shared their recollections of the time of the diagnosis and thereafter. In the vast majority of families, delays were experienced in receiving a diagnosis of FXS. Almost all parents described taking a proactive and persistent approach in searching for a diagnosis for their child's developmental delay and consulting multiple healthcare providers before the FXS testing was undertaken. Relatives described initial emotional reactions following the diagnosis of shock and grief which, over time, gave way to relief at having an explanation for the child's difficulties. In many cases, having a diagnosis empowered families. Families utilized a variety of coping strategies many of which demonstrated resiliency and assisted families to adjust to and make sense of the diagnosis of FXS in their family. These results provide a valuable insight into families' experiences of living with FXS and indicate the variety of ways in which a delayed diagnosis can impact a family. The findings will be considered with respect to genetic counseling practice and strategies for supporting families who have received a diagnosis of FXS will be discussed.

\section{NO NOVEL PATHOGENIC TCF4 AND MEF2C SEQUENCE VARIATIONS IDENTIFIED IN RTT PATIENTS}

R. Armani' ${ }^{1,2}$, N.Yang ${ }^{4}$, D. Cloosterman', J. Christodoulou ${ }^{1,2,3}$

'NSW Centre for Rett Syndrome Research, Children's Hospital at Westmead, Australia

${ }^{2}$ Discipline of Paediatrics and Child Health, Sydney Medical School,

University of Sydney, Australia

Department of Genetic Medicine, Sydney Medical School, University of Sydney, Australia

${ }^{4}$ Institute for Neuroscience and Muscle Research, Children's Hospital at Westmead, Australia

Rett Syndrome (RTT), now believed to be a disorder of neuronal plasticity, affects primarily females, and is characterized by an apparent normal development for the first 6-18 months of life followed by a loss of both fine and gross motor skills. Pathogenic mutations have been identified in MECP2 (Methyl-CpG Binding Protein) in classical and atypical RTT cases, in CDKL5 (CyclinDependent Kinase Like 5) in early onset atypical RTT patients and patients with epileptic encephalopathy, and in FOXGl (Fork-head Box $G 1$ ) in girls with the congenital RTT variant. Although mutations identified in the three genes to date have accounted for the majority of patients diagnosed with RTT, there are still a substantial number of patients with a RTT phenotype and no identifiable pathogenic mutation. In this study extensive screening of a large RTT cohort $(n=81)$ of $M E C P 2, C D K L 5$ and FOXG1 mutation negative patients was undertaken to identify novel pathogenic sequence variations in two new RTT candidate genes, TCF4 (Transcription Factor 4) and MEF2C (Myocyte Enhance Factor 2C). Mutation screening of the two candidate genes was performed through the use of high resolution melt, direct sequencing and diagnostic restriction digests. 
Our screening study did not identify any pathogenic sequence variations in the TCF4 and MEF2C gene in the patients cohort free of mutations in MECP2,CDKL5 and FOXG1, suggesting that these two genes are unlikely to account for a substantial proportion of mutation-negative RTT cases. The search for possible RTT candidate genes remains ongoing.

\section{TWO CASES OF PRENATALLY DETECTED I(18)(010)} WITH CONTRASTING OUTCOMES

\section{L. Baluyot, L. Carey \\ Genetics, Sydney IVF, Sydney, Australia}

We review 2 cases of i(18)(q10) detected at CVS by G banded karyotyping. Case 1: A 39 year old with DCDA twins, was referred due to AMA. A CVS was received from the lower right twin only and showed a 46,XX,i(18)(q10) karyotype in all cells examined. Fetal ultrasound at 19 and 20 weeks revealed no abnormalities and at 32 weeks an amniocentesis was performed. Rapid aneuploidy screening through QFPCR and uncultured FISH was consistent with a female fetus with no abnormalities detected. Cytogenetic analysis revealed a 46,XX female karyotype in all cells analyzed, suggesting the $\mathrm{i}(18)(\mathrm{q} 10)$ cell line is likely to be confined to the placenta. Case 2: CVS was received from a 39 year old referred due to a high risk combined nuchal translucency and 1st trimester biochemical screen consistent with trisomy 18. Rapid aneuploidy screening by QFPCR was consistent with a trisomy 18 female fetus. Cytogenetic analysis revealed a 46,XX,i(18)(q10) karyotype in all cells analyzed. On review, the single 18p STR marker assessed by QFPCR was homozygous, consistent with the i(18)(q10) karyotype. The pregnancy was terminated at 14 weeks gestation. Discussion: Whilst $\mathrm{i}(18)(\mathrm{q} 10)$ is a rare cytogenetic finding, trisomy 18 is seen in 1 in 8000 live births. The clinical phenotype of $\mathrm{i}(18)(\mathrm{q} 10)$ is consistent with full trisomy 18 with features including microcephaly, craniofacial dysmorphism, and congenital malformations. CPM has been reported in 1-2\% of CVS; however complete fetal-placental discordance is the extreme. Where an abnormal karyotype is reported (even with no normal cell line), and fetal abnormalities are not supportive of the diagnosis it is important to consider further fetal ultrasound and amniocentesis. A normal karyotype at amniocentesis is reassuring, however it does not rule out the possibility of fetal abnormality and therefore presents difficulties in genetic counseling.

\section{IMPACT ON TRUST AND ACTION WHEN DROWNING IN A SEA OF INCONSISTENT RISK PREDICTION GENERATED BY DTC GENETIC TESTING}

\section{K. Barlow-Stewart', C. Cormick ${ }^{3}$ \\ Centre for Genetics Education, NSW Health, Sydney, Australia \\ 2 Sydney Medical School, University of Sydney, Australia \\ Public Awareness and Community Engagement, Department of Innovation, Industry. Science and Research, Canberra, Australia}

Direct to consumer (DTC) genetic testing is being offered to Australians online by overseas companies whose laboratories are based in the US (23andMe and Navigenics) and Iceland (DeCODEme). Anecdotal evidence suggests that consumers who are early adopters of DTC are bringing the masses of data generated by these genome wide scans to genetics service providers for assistance with interpretation and advice. To investigate this potential impact, NSW and ACT based clinical geneticists and genetic counsellors were surveyed regarding the use of DTC testing over the last two years by current or new patients; possible reasons for its use; relevance and informativeness of the results; and how many GPs or other health professionals had sought advice where their patients had had DTC testing. To further understand the challenges facing consumers and genetics service providers with interpretation of the test results, the same person undertook DTC testing with the three companies. A diary was kept of his feelings and reactions during the testing process and following receipt of the result. The presentation will cover some of these including his apprehension while waiting for the results, particularly in relation to the conditions for which he had a family history; his reactions to dealing with the mass of risk data about so many health conditions generated, including analysis of how differently it was presented by the three companies; his initial perception that he may never need to see a doctor because it was all there; his confusion at receiving an increased risk for prostate cancer by two companies but a decreased risk by the third; who to go to for advice, when to seek expert advice, how to access genetic counseling, and what that may in turn trigger - as well as the crucial question of can genetic testing results be trusted?

\section{PRODUCING GENETICS EDUCATION RESOURCES FOR PRIMARY CARE PRACTITIONERS IS JUST THE BEGINNING: CHALLENGES IN DISSEMINATION AND ENGAGEMENT}

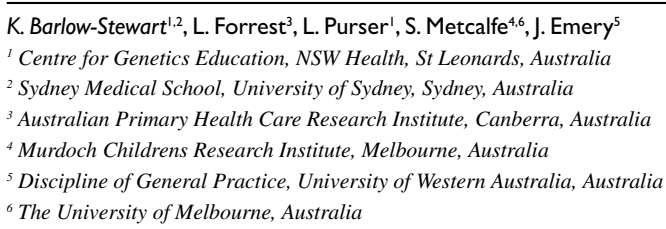

Genetics in Family Medicine: The Australian Handbook for General Practitioners (GPS) (http://www.nhmrc.gov.au/your_health/egenetics/ practitioners/gems.htm ) was developed by the Genetics Education in Medicine consortium for the Australian Government in both print and on-line formats (June 2007). A summary sheet (Genetics at a Glance $(\mathrm{G} @ \mathrm{G})$ ) contained guidelines for practice from the 17 modules, contacts for genetics services throughout Australia and a web-link to the full resource that was also available in hardcopy (\$A40). Promotion included a small launch at a national GP conference and a mail-out of the G@G with the October 2007 edition of the journal Australian Family Physician to every registered medical practitioner. Two years later, GPs working in an urban $(n=$ $690)$ in NSW and a rural and remote area $(n=70)$ in Queensland were approached to participate in a survey of the dissemination strategy and use and relevance of the resource to GPs' practice. While participation in the dissemination survey has been small (14\% RR), early findings confirm the consortium's concerns that the dissemination strategy and lack of associated educational activities has resulted in extremely limited awareness of the resource: $17 \%$ remember receiving G@G and 6\% (6/107) can still locate it, and only three of these GPs have accessed the full resource online or in print format. However, of the 68 urban and 9 regional GPs who opted into the learning activity based on use of the resource for two CPD points, the vast majority found the information useful or very useful in completing the activity and all the modules of information were rated highly as relevant to GP practice. Educational activities suggested by GPs essential to promote this valuable resource will be presented.

\section{PITT-HOPKINS SYNDROME ASSOCIATED} WITH TYPE 1 DIABETES AND ANORECTAL MALFORMATION IN A PATIENT WITH 18021.2 DELETION

\section{J. K. Nicholl'2, C. P. Barnett'}

${ }^{\prime}$ South Australian Clinical Genetics Service, Women's and Children's Hospital, Adelaide, Australia

${ }^{2}$ Cytogenetics Unit, Genetics and Molecular Pathology, SA Pathology at Women's and Children's Hospital, Adelaide, Australia

Background: Pitt-Hopkins syndrome (PTHS) is characterized by specific dysmorphic features (deep set eyes, broad-beaked nose, wide mouth with bow-shaped upper lip and widely spaced teeth), childhood-onset hyperventilation episodes, seizures, microcephaly, severe psychomotor retardation and a happy disposition. PTHS is caused by missense, nonsense, frameshift and splice-site mutations in TCF4 as well as deletions involving TCF4. Here we report on a 17-year-old girl with a deletion involving TCF4 with features of PTHS and previously unreported features of type 1 diabetes mellitus and a severe anorectal malformation. Case History: The patient was born at term following a normal pregnancy. The anus was markedly anteriorly displaced requiring serial dilatation and surgical correction at 6 months. 
She was referred to clinical genetics at age 5 because of dysmorphic features and severe psychomotor delay; all investigations were normal. Typical paroxysms of breathing and seizures have not been noted in this patient at any stage. At 8 years of age the patient developed acute ketoacidosis and was diagnosed with type 1 diabetes mellitus. At age 17 she was re-referred to clinical genetics and an oligoarray detected a $4.5 \mathrm{Mb}$ deletion involving 13 genes including TCF4 and a known susceptibility locus for type 1 diabetes (IDDM6). Literature review indicates two new features in our patient not previously described in PTHS caused by deletions, namely significant anorectal malformation and type 1 diabetes mellitus. In addition, the absence of breathing abnormalities or seizures in our patient supports recent evidence that breathing abnormalities and seizures appear to occur more commonly in PTHS patients with missense mutations. Deletion-type PTHS should not be discounted on the basis of absence of these features.

\section{DEXAMETHASONE NORMALIZES ABERRANT ELASTIC FIBRE PRODUCTION AND COLLAGEN 1 SECRETION IN LOEYS-DIETZ SYNDROME FIBROBLASTS: A POSSIBLE FUTURE THERAPY?}

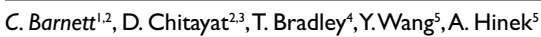 \\ ${ }^{I}$ South Australian Clinical Genetics service, Women's and Children's Hospital, Adelaide, \\ Australia \\ ${ }^{2}$ Division of Clinical and Metabolic Genetics, The Hospital for Sick Children, \\ University of Toronto, Canada \\ ${ }^{3}$ The Prenatal Diagnosis and Medical Genetics Program, Department of Obstetrics, \\ Mount Sinai Hospital; University of Toronto, Canada \\ ${ }^{4}$ Division of cardiology, The Hospital for Sick Children, University of Toronto, Canada \\ ${ }^{5}$ Division of Cardiovascular Research, The Hospital for Sick Children, \\ University of Toronto, Canada
}

Loeys-Dietz syndrome is an autosomal dominant connective tissue disorder characterized by facial dysmorphism, cleft palate, dilation of the aortic arch, blood vessel tortuousity and a high risk of aortic dissection. It is caused by mutations in the transforming growth factor beta receptor 1 and 2 (TGFBR 1 and TGFBR2) genes. Fibroblasts derived from 12 Loeys-Dietz syndrome patients, six with TGFBR1 mutations and six with TGFBR2 mutations, were analyzed using RT-PCR, biochemical assays, immuno-histochemistry and electron microscopy for production of elastin, fibrillin 1, fibulin 1 and fibulin 4 and deposition of collagen type I. All Loeys-Dietz syndrome fibroblasts with TGFBR1 mutations demonstrated decreased expression of elastin and fibulin 1 genes and impaired deposition of elastic fibres. In contrast, fibroblasts with TGFBR2 mutations consistently demonstrated intracellular accumulation of collagen type 1 in the presence of otherwise normal elastic fibre production. Treatment of the cell cultures with dexamethasone induced remarkable up-regulation in expression of tropoelastin, fibulin 1 and fibulin 4-encoding mRNAs leading to normalization of elastic fibre production in fibroblasts with TGFBR 1 mutations. Treatment with dexamethasone also corrected the abnormal secretion of collagen type 1 from fibroblasts with TGFBR2 gene mutations. Since the organogenesis-relevant elastic fibre production occurs exclusively in late fetal and early neonatal life, this time-line raises the intriguing question of whether our in vitro results could be replicated in pregnancy by dexamethasone treatment of women known to be carrying fetuses with LDS. Dexamethasone already has a proven track record in the prenatal treatment of congenital adrenal hyperplasia. Further studies are required to determine if dexamethasone treatment of fetuses prenatally diagnosed with Loeys-Dietz syndrome would prevent or alleviate the connective tissue and vascular defects seen in this syndrome.

\section{LATE-ONSET VARIANT FIBRODYSPLASIA OSSIFICANS PROGRESSIVA LEADING TO MISDIAGNOSIS OF ANKYLOSING SPONDYLITIS}

C. P. Barnett', M. Dugar' ${ }^{2}$, E.A. Haan'

'South Australian Clinical Genetics service, Women's and Children's Hospital, Adelaide, Australia

${ }^{2}$ Department of Rheumatology, Royal Adelaide Hospital, Adelaide, Australia

Background: Fibrodysplasia ossificans progressiva (FOP) is a rare (1 per 2 million) condition characterized by childhood-onset extra-skeletal heterotopic bone formation in the soft tissues, leading to severe disability. Patients classically have congenital malformation of the great toes (hallux valgus/malformed first metatarsals/monophalangism). A recurrent mutation in the ACVR1 gene, a gene encoding a type 1 bone morphometric protein (BMP) transmembrane receptor, cause most cases of FOP. In this report we describe a woman with variant FOP with normal great toes and late onset heterotopic ossification, who was misdiagnosed with ankylosing spondylitis for ten years and unnecessarily treated with sulfasalazine and methotrexate. Case History: The patient presented at 21 years of age with a 'stiff back'. Plain x-ray of the lumbar spine was initially normal and after extensive investigation non-specific spondyloarthropathy was diagnosed. Sulfasalazine treatment was commenced. Three years later, the patient developed a painless mass on the inferior border of the mandible. The mass resolved completely over 3 months with no remaining clinical or radiological evidence of the lesion. A similar self-resolving lesion adjacent to the thyroid gland occurred three years later. Worsening back pain led to further imaging which showed extensive ankylosis and new bone formation extending from L3-S1. Ankylosing spondylitis was diagnosed and methotrexate was added with little symptomatic improvement. Three years later a CT scan of the entire spine was performed which revealed extensive ossification of the ligamentum flavum in the thoracic spine and interspinous ligament ossification in the lumbar spine, suggestive of FOP. Both first toes were clinically and radiologically normal. The patient was referred to clinical genetics and sequencing of the ACVR1 gene revealed heterozygosity for a non-recurrent mutation $(\mathrm{c} .605 \mathrm{G}>\mathrm{T})$ in ACVR1. This case highlights the importance of considering FOP in patients with ankylosing spondylitis, even without the characteristic toe abnormalities seen in classic FOP.

\section{DRAYER'S SHORT STATURE SYNDROME: REPORT OF AN ADDITIONAL CASE OF 15026 MICRODELETION INVOLVING IGF1R AND REVIEW OF THE LITERATURE}

\author{
L. I. Rudaks', J. K. Nicholls², D. Bratkovic', C. P. Barnett' \\ 'South Australian Clinical Genetics Service, Women's and Children's Hospital, \\ SA Pathology, Adelaide, Australia \\ ${ }^{2}$ Cytogenetics Unit, Genetics and Molecular Pathology, SA Pathology at Women's \\ and SA Pathology, Adelaide, Australia
}

Background: Terminal microdeletion of the long arm of chromosome 15 is a rare cause of short stature, with only a few cases reported to date. Most cases have been associated with pre- and postnatal growth restriction, microcephaly, developmental delay and various skeletal and facial dysmorphic features (Drayer's syndrome). The gene within the 15q26.3 region responsible for growth restriction is the insulin-like growth factor 1 receptor gene (IGF1R, OMIM 147370), a gene that plays a role in skeletal growth, brain development and carbohydrate metabolism. Case report: We report on a patient with a $4.07 \mathrm{Mb} 15 \mathrm{q} 26.2$-q26.3 terminal deletion, diagnosed using Agilent Technologies $60 \mathrm{~K}$ oligonucleotide array, presenting with speech delay, subtle dysmorphic features and short stature. The patient was born in China at 36 weeks gestation with a birth weight of $1.9 \mathrm{~kg},(<3 \mathrm{rd}$ percentile) and although birth length was not recorded he was noted to be very short. When seen at 4 years and 5 months his height was $90 \mathrm{~cm}(4 \mathrm{~cm}$ below the $1 \mathrm{st}$ centile), weight $14.5 \mathrm{~kg}$ (3rd-10th centile) and head circumference $49 \mathrm{~cm}$ ( $25 \mathrm{th}$ centile). He had down-turned corners of the mouth, a thin upper lip, hypoplasia of the alae nasi (not previously described), clinodactyly 
of the fifth fingers bilaterally, brachydactyly, short nails and an undescended testis. At 4 years of age he had a vocabulary of less than 20 words and was not constructing sentences but had only mildly delayed motor milestones. Discussion: Normal intrauterine and postnatal growth is dependent upon appropriate insulin-like growth factor 1 (IGF-I) and IGF1R function. Deletions involving IGF1R at $15 \mathrm{q} 26.2$ are a very rare cause of pre- and postnatal growth restriction that is potentially responsive to growth hormone therapy. Our case adds to the few cases with deletions encompassing IGF1R described in the literature and further clarifies the phenotypic picture of this emerging microdeletion syndrome.

\section{NON-CODING ELEMENTS THAT REGULATE MECP2 EXPRESSION}

E. Bettella ${ }^{1,2}$, G. Ho ${ }^{1,3}$, A. Murgia ${ }^{2}$, J. Christodoulou ${ }^{1,3}$

Centre for Rett Syndrome Research, Children's Hospital at Westmead, Australia 'Department of Paediatrics, University of Padua, Italy

Disciplines of Paediatrics \& Child Health and Genetic Medicine, University of Sydney, Australia

Rett syndrome (RTT, OMIM 312750) is a severe X-linked neurodevelopmental disorder with onset during early childhood and with an incidence of approximately $1 / 8,000$ females by the age of $15 \mathrm{yrs}$. Mutations in the methyl CpG-binding protein 2 (MECP2, OMIM \#300005) gene are found in up to $95 \%$ of classical RTT cases. However, a significant number of RTT patients have no identified mutations in $M E C P 2$ or other genes associated with RTT, including $C D K L 5$ and FOXG1. We speculate that sequence variations in 3'UTR/polyadenylation signals of the $M E C P 2$ gene may lead to alterations in gene regulation. Characterization of the MECP2 3'UTR in these patients would potentially highlight the role of non-coding regions in the regulation of its translation. We are currently measuring the variation in $M E C P 2$ mRNA levels in $M E C P 2, C D K L 5$ and FOXG1 mutation-negative RTT patients by quantitative PCR. Those patients with reduced $M E C P 2$ mRNA levels will then be screened for sequence variations in the 3'UTR. Performing an in silico analysis, we have identified the putative regulatory region of the human $M E C P 2$ gene. We have generated a luciferase reporter vector driven by the human MECP2 promoter and containing the $1800 \mathrm{bp} \mathrm{3'UTR}$ downstream of the luciferase coding sequence. Using this expression system, we aim to examine the effect of sequence variations in the MECP2 3'UTR by performing in vitro transfection experiments using non-neuronal (COS-7) and neuronal (SH-SY5Y) cell lines. The 3'UTR is known to play an important role in mRNA stability/degradation, nuclear export, subcellular localization and translational efficiency. The MECP2 3'UTR is one of the longest UTRs documented and variations in this region have been shown to alter mRNA levels in autistic patients. We plan to explore whether differences in expression levels of $M E C P 2$ could potentially be attributed to the creation of new target sites for specific miRNAs.

\section{GENETIC COUNSELLORS' PERCEPTIONS OF THE BLURRED BOUNDARIES BETWEEN CLINICAL RESEARCH AND CLINICAL CARE}

\section{R. Bogwitz}

Familial Cancer Centre \& Genetics, Royal Melbourne Hospital, Australia

As genetics embeds itself in mainstream medicine, the degree of specialisation of genetic counsellors is growing and we are finding ourselves with increasing opportunities to be involved in research as part of our everyday clinical care. We recognise that research improves our clients' lives, and that our involvement in research helps to develop genetic counseling as an independent profession. Research and clinical care are theoretically distinct due to their differing motivations, aims, and outcomes. However in practice, these activities can become blurred in the eyes of both the clients and the genetic counsellors, creating a sense of unease for both groups for differing reasons. I aimed to explore practising genetic counsellors' experiences in introducing clients into, or actively recruiting them for, clinical research. I was interested in uncovering some of our apprehensions about this process in the hope that our awareness of the issues can help us distinguish these two parts of our roles, enhancing the overall care for the client in an ethical and responsible way. Approximately 150 ASGC genetic counsellors were emailed an invitation to complete an online survey. The survey consisted of 10 multiple choice or matrix of choice questions and each included opportunity for comments. 87 Genetic counsellors responded from all states and territories in Australia (except NT) and from New Zealand. Results were compiled and are displayed as percentages in pie, bar, and line charts as appropriate. Some of the concerns include the appropriateness of timing of recruitment (soon after or during clinical care sessions), the feelings of coercion associated with development of client rapport, the ethical dilemmas associated with having incentives to recruit clients to projects, and being unable to ensure our clients are aware of the distinction between the clinical and research activities.

\section{CANCER IN MY FAMILY (CIMF): A NEW ONLINE SUPPORT} GROUP FOR PEOPLE WITH FAMILIAL CANCER RISK

M. Bogwitz ${ }^{1,4}$, K.Wakelin 2,4 J. Jenkins ${ }^{3,4}$, T. McArdle ${ }^{5}$,A. Spillare ${ }^{2}$, T.Waters $^{2}$, H. Lane ${ }^{3}$, M. Black, M. Cameron ${ }^{2,4}$

${ }^{I}$ Familial Cancer Centre \& Genetics, Royal Melbourne Hospital, Australia

${ }^{2}$ Cancer Information \& Support Service, Cancer Council Victoria, Melbourne, Australia

${ }^{3}$ Consumer Representative, Victorian Cooperative Oncology Group (VCOG),

Cancer Council Victoria, Melbourne, Australia

${ }^{4}$ Victorian Cooperative Oncology Group (VCOG), Cancer Council Victoria, Melbourne, Australia

${ }^{5}$ Victorian Family Cancer Register (VFCR), Cancer Council Victoria, Melbourne, Australia

Being at risk of carrying a genetic pre-disposition or having a strong family history of cancer presents many issues. These may include decision making about genetic testing, family relational issues, anxiety related to ongoing screening, implications for life or health insurance, and making decisions about prophylactic surgery such as mastectomy. Currently, people who have been identified to have an increased risk of developing cancer have several levels of accessible support. They can register with the Victorian Family Cancer Register, access several printed and online resources or re-initiate contact with a familial cancer centre. Other sources of support include Facebook and Yahoo online groups, however privacy can often be an issue with such sites. With regard to psychosocial support, people are of course able to contact familial cancer centres, but these services are unable to provide peer-to-peer support that some people need. Those who have been tested and found to carry a cancer susceptibility gene are able to access the Cancer Connect (telephone based) Gene Support Program. This service does not include people who are unable to be tested who may have significant risk or unmet support needs. It also cannot assist people who are going through the difficult process of deciding whether or not to be tested. We formed a working party to facilitate the formation of a new online support group, hosted via the www.cancerconnections.com.au website. This is an online community, moderated by Cancer Council staff. This group is available to anyone with a high risk of cancer because of their family history. Potential participants are assessed at an individual level to ensure appropriate membership. We hope that this group will assist in providing for the support needs for people with an increased risk of cancer. www.cancerconnections.com.au is an initiative of Cancer Council New South Wales.

\section{A STUDY OF THE BODY COMPOSITION OF CHILDREN AND ADOLESCENTS WITH PHENYLKETONURIA (PKU) \\ C. M. Bonifant', M. S.White', P. S. Davies', J. J. McGill ${ }^{3}$ \\ ${ }^{I}$ Nutrition and Dietetics Department, Royal Children's Hospital, Brisbane, Australia ${ }^{2}$ Children's Nutrition Research Centre, Brisbane, Australia \\ ${ }^{3}$ Department of Metabolic Medicine, Royal Children's Hospital, Brisbane, Australia}

PKU is treated with a low protein diet and supplementation with a phenylalanine-free, formula for life. Growth impairment has been described even in patients with a total protein intake at or above the recommended allowance and there is conflicting information about 
the body composition of patients with PKU in the literature. Huemer et al and Allen et al report no difference in fat mass or fat free mass in a group of children and adolescents with PKU compared with healthy controls, while Alberson et al. reports an increased percentage of body fat in older girls with PKU compared with healthy controls. It is unknown if simple anthropometric measures are indicative of body composition in this group. This study aims to determine if children and adolescents with PKU have an altered body composition in comparison with existing normative data, and to compare skinfold thickness against body fat measures via air displacement plethysmography (Bod Pod) measurements to determine possible bias. Twenty children and adolescents were identified for the study. All participants had anthropometric measurements taken and underwent body composition testing (Bod Pod, Total Body Potassium, skinfold testing). These measures were compared with a normative data set using matched controls and body fat determined by skin fold thickness and the Bod Pod was compared. Upon preliminary analyses both TBK $z$ score and percentage body fat are significantly lower $(p<.05)$ in patients with PKU. Further analyses with the full data set will be presented. By identifying the body composition using several methods of body composition methodology, and validating simple, noninvasive measures of body composition, it is hoped that practitioners without access to sophisticated body composition equipment will be able to accurately identify fat mass in this group, enabling more precise and nutritionally adequate dietary prescription.

\section{AN EXPLORATION OF HEALTH PROFESSIONALS VIEWS ON PROVIDING A GENETIC COUNSELING SERVICE TO FAMILIES WITH AN UNEXPECTED POSTNATAL DIAGNOSIS OF DOWN SYNDROME}

\section{S. L. Borrie', E. Muggli ${ }^{3}$, J. Hodgson', C. McAlpine ${ }^{2}$ \\ ${ }^{\prime}$ Genetic Health Services Victoria, Murdoch Children's Research Institute, Melbourne, Australia \\ ${ }^{2}$ Down Syndrome Association of Victoria, Melbourne, Australia \\ ${ }^{3}$ Public Health, Murdoch Children's Research Institute, Melbourne, Australia}

Background: In Victoria, Australia, approximately 60 children are born with Down syndrome each year. This occurs against a backdrop of shifting maternal age distribution and widespread access to prenatal screening and testing. It is well described that families with a baby receiving a diagnosis, such as Down syndrome, undergo a period of grief and adaptation while adjusting to their 'new' life path. Provision of support for families during this time has been shown to improve their adaptation and adjustment to the diagnosis. One of the suggested professions to support families in hospital following diagnosis of Down syndrome is genetic counseling. Methods: To explore the opinions and beliefs of health professionals currently involved in supporting families when a baby is diagnosed with Down syndrome, either prenatally or after birth, about the utility of genetic counseling following a postnatal diagnosis. A total of 7-10 health professionals were recruited for this study, each undergoing in-depth interviews of approximately 45 minutes. Thematic analysis was undertaken on de-identified transcripts. Results: Differing interpretations of the role of a genetic counsellor, in particular the demarcation between prenatal and paediatric as well as health service provider differences were found to be important factors in deciding whether genetic counseling may be appropriate in supporting families following a postnatal diagnosis of Down syndrome. Discussion: A unified approach amongst health professionals may increase the level of support provided to families when a baby is diagnosed with Down syndrome after birth.

\section{COEXISTENCE OF THE PHILADELPHIA CHROMOSOME AND INVERSION 16 DURING BLAST CRISIS IN CHRONIC MYELOID LEUKAEMIA: A CASE REPORT ON THIS RARE OCCURRENCE}

\section{T. Bowen',V.Yagnesh', G. Romeo', J. O'Reilly',A. McQuillan ${ }^{2}$}

${ }^{\prime}$ Haematology, Pathwest Laboratory Medicine, Royal Perth Hospital, Perth, Australia

${ }^{2}$ Haematology, Saint John of God Pathology, Perth, Australia

We report a rare case of chronic myeloid leukaemia (CML) in chronic phase with the classic $\mathrm{t}(9 ; 22)(\mathrm{q} 34 ; \mathrm{q} 11.2)$ accompanied by the $\operatorname{inv}(16)(\mathrm{p} 13.1 \mathrm{q} 22)$, in a 54 year old lady, approximately 18 months post diagnosis and several weeks before she rapidly progressed to acute myeloid leukemia (AML). The patient was initially treated with imatinib $400 \mathrm{mg}$ daily and monitored by quantitative PCR (Q-PCR). The patient did not achieve a $3 \mathrm{log}$ reduction of their BCR-ABL transcript levels at 12 months on imatinib and the dose of imatinib was increased to $600 \mathrm{mg}$ daily. At 18 months post diagnosis, a 4 fold increase in the BCR-ABL transcript levels was observed in the peripheral blood. The presence of the $\mathrm{t}(9 ; 22)$ and inv 16 was confirmed by cytogenetics and fluorescence in situ hybridisation (FISH) in the bone marrow. Although morphologically still in chronic phase at this time, the patient soon became neutropenic and thrombocytopenic with myeloid blasts in the peripheral blood. The patient received induction chemotherapy but developed acute respiratory failure and required two weeks in the intensive care unit. The patient is now on nilotinib and is in hematological remission with a falling BCR-ABL transcript level and a cytogenetically normal karyotype. Cytogenetics, FISH and Q-PCR used together to monitor this patient provided an early indication of imminent progression of CML and a superior means of monitoring disease response to therapy. Although rare, this case provides evidence that the emergence of inv(16), as a secondary abnormality in CML, leads to the rapid progression to accelerated phase and blast crisis and is not associated with a favourable prognosis, as seen in de novo AML.

\section{WHEN OCCAM'S RAZOR FAILED CANCER GENETICS: BIRT HOGG DUBÉ AND ATTENUATED FAP IN THE SAME KINSHIP}

\section{J. Burgess', M. J. Field ${ }^{2}$}

${ }^{2}$ Clinical Genetics Service, Austin Health, Melbourne, Australia

${ }^{2}$ Department of Clinical Genetics, Royal North Shore Hospital, Sydney, Australia

Our consultand, a 59-year-old women, self-referred for predictive testing for Birt-Hogg-Dubé syndrome (BHDS). Her sister (the proband) was diagnosed with BHDS after presenting with a renal malignancy and fibrofolliculomas. BHDS is an autosomal dominant condition predisposing to various cutaneous manifestations, pulmonary cysts \& pneumothoraces and renal tumours. Colorectal tumour risk may be elevated. BHDS is caused by mutations in the FLCN gene (chromosome 17). Our client had had bowel adenomas removed over a 20 -year period $(>10)$. She had clinical features of BHDS. The family also had a history of colorectal cancer and this was believed to be linked to BHDS. Her genetic result revealed she had inherited her family's mutation (FLCN c.1285delC). Occam's razor states when diagnosing a given illness or disease, doctors should strive to look for the fewest possible causes that will account for all symptoms. Subsequently, her 37-year-old son presented for predictive testing for BHDS. He was found to be gene negative for the BHDS mutation but had multiple adenomas detected during a colonoscopy $(>30)$. He subsequently underwent mutation testing for familial adenomatous polyposis (FAP). Familial Adenomatous Polyposis is an autosomal dominantly inherited cancer predisposition syndrome in which hundreds of precancerous colonic polyps develop. Without colectomy, colon cancer is inevitable. Attenuated FAP has a significant risk of colon cancer but fewer colonic polyps and diagnosis of colon cancer at a later age. Both classical and attenuated FAP is caused by mutations in the $A P C$ gene (chromosome 5). The 37-year-old son was found to have a maternally inherited $A P C$ mutation $(A P C$ c. $412 \mathrm{G}>\mathrm{T}$ ) giving rise to attenuated FAP. We believe 
this is the only reported case of a family with the dual diagnoses of BHD and FAP where both conditions are inherited independently.

THE IMPORTANCE OF RNA ANALYSIS IN INTERPRETING A NOVEL SPLICE MUTATION

\section{Calabro, D. Du Sart}

Molecular Genetics, VCGSPathology, Murdoch Childrens Research Institute, Melbourne, Australia

Hereditary non-polyposis colorectal cancer (HNPCC) is a dominantly inherited cancer syndrome characterized by familial accumulation of early onset colorectal, endometrial and other extracolonic tumours. HNPCC is caused by germline mutations in the mismatch repair (MMR) genes MLH1, MSH2, MSH6 and PMS2. Several molecular techniques are used to screen the various MMR mutation types, which include truncating, missense, deletion mutations or variations in splice regulatory sites. The difficulty in HNPCC genetic testing is interpreting the pathogenicity of these mutations. A patient presented with colorectal cancer at 31 years of age and breast cancer at 37 years of age. There was no significant family history of breast or colorectal cancer; however the tumour samples presented with low microsatellite instability and absent immunohistochemical staining of the MSH2 and MSH6 genes. Gene sequencing identified a splice site mutation in intron 15 of the MSH2 gene $\{$ NM_000251.1\}:c.2635-1G >A and no mutations in the MSH6 gene. The MSH2 mutation occurs at the highly conserved, invariant splice site sequence. A similar mutation at the same nucleotide position was listed on the HNPCC database which predicted a possible effect on RNA splicing. In silico splicing software analysis was inconsistent, with suggestions that this mutation may or may not alter splicing. Testing was offered to other family members on the basis that RNA analysis was in progress. However, another interstate genetics service questioned the pathogenicity of this mutation as the splicing would involve the last exon of the gene, indicating that further testing was required before this result could be used for predictive testing. We will present methods used to detect splicing in the terminal region of the gene, in order to classify the pathogenicity of the mutation.

\section{IDENTIFICATION OF NUCLEAR LOCALISATION SIGNAL SEQUENCES IN ADENOVIRAL TERMINAL PROTEIN}

V.A. Chailertvanitkul, C.W. Pouton

Medicinal Chemistry and Drug Action, Monash University, Australia

The future of gene therapy is dependent on the development of vectors that can selectively and efficiently deliver therapeutic DNA to target cells in vivo with minimal toxicity. Virus vectors are the most widely used transducing systems. However, there are many ethical and safety concerns associated with the use of viruses in humans. Non-viral vectors are more suitable because of the lack of a specific immune response; but these are currently inefficient due to poor gene transport. There is a widespread interest in the use of adenoviruses (Adv) primarily because of their ability to efficiently deliver doublestranded DNA to the nucleus. In addition, their large genome allows for extensive modification and incorporation of therapeutic genes. The basic understanding of how Adv nuclear delivery systems work is fundamental to ensure further development and enhancement of the non-viral gene therapy. As part of an investigation into the mechanisms of Adv nuclear delivery we are examining the role of the Adv Terminal Protein (TP), a protein that is covalently coupled to the 5' ends of the Adv DNA. Previous research suggested that the TP encodes the nuclear localisation signal (NLS). However, the specific encoding sequence has not been identified. To identify the encoded NLS, PCR-based approaches were used to amplify several truncated TP derivatives and to create site-directed TP mutants. The products were then inserted into mammalian expression plasmids pcDNA6.2/C-EmGFP (fused in frame to the C-terminus of the GFP) and pcDNA6.2/N-YFP (fused in frame to the N-terminus of the YFP) using the Gateway recombination system (Invitrogen). Transfection of mammalian cells (HeLa and COS-7 cells) allowed evaluation of the extent of nuclear delivery of each fusion protein by determining the nuclear/cytoplasmic fluorescence ratio $\mathrm{F}(\mathrm{n} / \mathrm{c})$. Using this method, the fusion protein encoding the NLS was identified.

\section{PRENATAL DIAGNOSIS OF BALANCED AND UNBALANCED TRANSLOCATIONS USING THE AFFYMETRIX ${ }^{\circledR}$ CYTOGENETICS WHOLE-GENOME 2.7M ARRAY}

J. Chan', C. Chua', P. L. Ng', S. Chan',V.Toh', A. Biswas'²,Y. C.Wong², L. Gole

${ }^{I}$ Cytogenetics Laboratory, Dept. of Laboratory Medicine, National University Health System, Singapore, Singapore

${ }^{2}$ Dept. of Obstetrics and Gynaecology, National University Health System, Singapore, Singapore

Interchromosomal rearrangements in which there are balanced exchanges of genetic material between two or more chromosomes are called reciprocal translocations whereby no loss or gain of any genetic material occurs. In the general human population, approximately 1 in 1000 individuals is a balanced translocation carrier. While these carriers are phenotypically normal, they are at risk for having miscarriages or abnormal offspring. Here we present two prenatal cases of translocation in which one parent was a known carrier of a reciprocal translocation. The first case was from amniotic fluid culture showing an apparently balanced translocation of 46,XX,t(1;12)(q42;q24.3)pat; the carrier of the translocation being the father. the second case was also from amniotic fluid cells which showed an unbalanced translocation giving rise to a karyotype of 46,XY,der(6)t(6;18)(p25;q21.3)mat; the mother being the carrier of the translocation. In both cases there was past history of children with multiple anomalies and abnormal karyotypes. DNA extracted from these amniotic fluid cells was analyzed using the Affymetrix ${ }^{\circledR}$ Cytogenetics Whole-Genome $2.7 \mathrm{M}$ Array high resolution SNP microarray. The first case study, the microarray analysis showed no gain or loss of any genetic material and hence confirmed that the translocation was balanced as seen in the karyotype. As for the second case study, the analysis showed that there was a loss of genetic material that corresponded to the region of $6 \mathrm{p} 25.1$ to 6 pter and a gain of genetic material that corresponded to the region of $18 \mathrm{q} 21.31$ to $18 \mathrm{qter}$. Interestingly, in addition to the expected pattern, the analysis also showed a gain of genetic material corresponding to the region of $18 \mathrm{p} 11.23$ to $18 \mathrm{p} 11.31$ that was not detected in the karyotype. These results demonstrated that high-resolution microarray platforms allow in-depth assessment of chromosomal aberrations or translocations.

\section{FLT3 AND NPM1 TESTING IN CYTOGENETICALLY NORMAL AMLS - A LABORATORY EXPERIENCE}

M. Chauhan, A. Mitra, G. Mullan, P. Dalzell, M. Caramins, M.Y.Yip

Molecular and Cytogenetics Unit, SEALS, Prince of Wales Hospital, Sydney, Australia

Acute myeloid leukaemia (AML) encompasses a broad range of disorders that are all characterized by an arrest of maturation along with uncontrollable proliferation of hematopoietic progenitor cells. A number of recurrent structural and numerical cytogenetic aberrations have been identified that serve as diagnostic and prognostic markers. However, the largest subgroup of AML patients $(\sim 40 \%)$ have no identifiable cytogenetic abnormalities. Cytogenetically Normal (CN) AML has been more closely examined in recent years, and a number of molecular mutations have been identified including NPM1, FLT3, CEBP $\alpha$, and MLL. CN AMLs have previously been reported with an intermediate prognosis. Two new provisional entities - AML with mutated NPM1 and AML with mutated CEBPA - have been included in the 2008 WHO classification of AML. The nucleophosmin gene NPM1 has been found to be mutated in $50-60 \%$ of patients with CN AML. The NPM1 protein is involved in regulation of the ARF-p53 tumour-suppressor pathway and the most frequent mutation in the gene is a 4-nucleotide insertion. This mutation in isolation is associated with a favourable prognosis. Mutations of the Fms-like tyrosine kinase 3 gene (FLT3) have been found to be strongly correlated with an adverse outcome in patients with AML. The most common mutation - an inframe Internal Tandem Duplication (ITD) is seen in $28-33 \%$ of CN AMLs. This laboratory has offered mutation 
testing for FLT3 and NPM1 since August 2008. Here we present our findings and compare the frequency of mutations with those reported in recent literature. To date our cohort has an abnormality rate of $60 \%$, demonstrating the importance of molecular testing in cytogenetically normal AMLs.

\section{UNFOLDING COMPLEXITIES IN LQT SYNDROME TESTING \\ B. Chong', D. Phelan', I. Macciocca'2, P. James², D. Du Sart' \\ ${ }^{\prime}$ Molecular Genetics, Victorian Clinical Genetics Services Pathology, Murdoch Childrens Research Institute, Melbourne, Australia \\ ${ }^{2}$ Clinical Services, Victorian Clinical Genetics Services, Murdoch Childrens Research Institute, Melbourne, Australia}

Long QT syndrome (LQTS) is a familial cardiac disorder that is associated with a prolonged QT segment on the ECG and polymorphic ventricular arrhythmias. These arrhythmias often occur in relation to exercise or emotional stress and may result in recurrent syncope, seizures, or sudden cardiac death. The prevalence of LQT is at least 1 in 5000 and may be up to 1 in 1000 . LQTS has been recognized as mainly Romano-Ward syndrome (an autosomal dominant from) and Jervell and Lang-Nielsen (JLN) syndrome (an autosomal recessive form). Two other more rare syndromes have also been described, namely, Andersen syndrome and Timothy syndrome. Defects in 12 genes $K C N Q 1$ (LQT1), KCNH2 (LQT2), SCN5A (LQT3), ANK2 (LQT4), KCNE1 (LQT5), KCNE2 (LQT6), KCNJ2 (LQT7), CACNA1C (LQT8), CAV3 (LQT9), SCN4B (LQT10), AKAP9 (LQT11), SNTA1 (LQT12) have so far been shown to cause LQT. The accepted practice currently for testing patients is sequencing of LQT 1, 2, 3, 5 and 6 genes. Sequencing will identify point mutations, small insertions or deletions but does not detect large deletions. To date a number of sequence variations and some large deletions have been identified. In addition, there are reports of about $20 \%$ compound heterozygocity of mutations within the same or in different genes. Therefore, the assignment of pathogenicity of the sequence variations is complex and many clinical implications arise regarding predictive testing and the counseling of at risk family members. We show examples of families with autosomal dominant LQT syndrome with more than one variant, an exon deletion and a variant in the rarer LQT7 gene. As an outcome, we propose a strategy for the management of LQT testing.

\section{POPULATION-BASED CARRIER SCREENING FOR CYSTIC FIBROSIS IN VICTORIA: \\ THE FIRST THREE YEARS EXPERIENCE}

B. Chong',A. Ritchie', S. Pantaleo', M. Chow', B. Ong'2, J. Massie', V. Petrou', D.Amor ${ }^{4}$ D. Du Sart'

${ }^{I}$ Molecular Genetics, VCGS Pathology, Murdoch Childrens Research Institute, Melbourne, Australia

${ }^{2}$ Scientific Services, Corporate Services, Murdoch Childrens Research Institute, Melbourne, Australia

${ }^{3}$ Respiratory Medicine, Victorian Clinical Genetics Services, Murdoch Childrens Research Institute, Melbourne, Australia

${ }^{4}$ Clinical Services, Victorian Clinical Genetics Services, Murdoch Childrens Research Institute, Melbourne, Australia

Cystic fibrosis (CF) is the most common inherited, lifeshortening condition affecting Australian children. The main clinical manifestations are chronic suppurative lung disease, pancreatic exocrine insufficiency and elevated sweat electrolytes. Although treatments have improved over the two decades since the CFTR gene was discovered there is still no cure. Most children survive to adulthood but the treatments are complex and there are many years of poor health. Genetic testing for CF has been incorporated into newborn screening programs for the early identification of affected individuals, and reproductive choices offered to parents for subsequent pregnancies. However, it is possible to offer carrier testing before a couple has an affected infant. While over 1500 CFTR gene mutations and polymorphisms have been described, the majority of carriers (80\%) can be detected with a panel of 12 mutations. The inheritance of CF is autosomal recessive and carriers are completely healthy. Carrier screening for CF was recommended in the USA in 2001 and subsequently there has been a $50 \%$ reduction in the incidence of affected infants. In the absence of a government-supported program, we initiated a fee-for-service population-based CF carrier screening program in Victoria, Australia, in 2006. Testing in our laboratory was initially carried out using a SNP based assay and now using MALDI-TOF mass spectrometry. Mass spectrometry based technology (SEQUENOM) combines the sensitivity of PCR and the accuracy of Matrix-Assisted Laser Desorption/Ionization Time Of Flight Mass Spectrometry (MALDI-TOF MS) for measuring the amount of genetic target material and the derived variants. This technology can be applied to the detection of single nucleotide polymorphisms (SNPs) by differentiating between their fragment masses, with a high degree of multiplexing capability and is amendable to high throughput analyses. We present our experience of the first three years of carrier screening for CF and the use of the MALDI-TOF technology.

\section{EVALUATION OF PATIENTS' EXPERIENCES OF A TELEPHONE GENETIC COUNSELING APPOINTMENT}

\section{Cicciarelli, A. Lewis, C. Michael-Lovatt, P. Chan, M. Young, G. Mitchell}

Peter MacCallum Familial Cancer Centre, Melbourne, Australia

Telephone genetic counseling appointments commenced at the Peter MacCallum Familial Cancer Centre in April 2009. This service was initiated to provide patients with an alternative way to receive an assessment of their familial risk of developing cancer and so provide a more flexible, patient-centred approach as well as reduce patient waiting times for appointments. Telephone appointments are intended predominately for people whose known family history of cancer indicates they do not have a high risk of developing cancer or whose case requires additional work up before attending a face to face clinic appointment. To evaluate our patients' experience of a telephone genetic counseling appointment, a questionnaire was developed and posted to the 152 patients who had received a telephone genetic counseling appointment in the period between April 2009 and March 2010. The questionnaire explored:

- how the patient perceives receiving medical information via telephone

- the patient's perceived ability to express their thoughts, worries and concerns over the telephone

- identifying areas of improvement to aid the continued development of the telephone clinic

Of the 152 patients, $93(61 \%)$ returned the questionnaire. Telephone appointments were well received with patients citing convenience as the main benefit of this type of appointment. Patients felt they were still receiving a personalized service where they were able to speak openly about their thoughts, worries and concerns even though the discussion was over the telephone. A telephone appointment allowed access for patients who may not have been able or willing to attend for a face-to-face appointment and the prearranged time allowed people to plan their day and find a suitable location for the call. We will continue our analysis of this study to try and identify additional areas of improvement.

\section{NUP214-ABL1 POSITIVE T-CELL ACUTE LYMPHOBLASTIC LEUKAEMIA PATIENT SHOWS A FAVOURABLE RESPONSE TO IMATINIB THERAPY POST-RELAPSE}

S. Clarke, J. O'Reilly, G. Romeo, J. Cooney

Department of Haematology, PathWest Laboratory Medicine WA, Royal Perth Hospital, Perth, WA, Australia

Recent studies have shown the presence of the NUP214-ABL1 fusion gene in $6 \%$ of cases of T-cell acute lymphoblastic leukemia (T-ALL). Like the BCR-ABL1 fusion gene seen in cases of chronic myeloid leukemia, the NUP214-ABL1 fusion gene encodes a constitutively activated tyrosine kinase. Given that tyrosine kinase inhibitors (TKIs) suppress the constitutive kinase activity of ABL1, there is potential for their use in the treatment of patients with NUP214-ABL1 positive T-ALL. However, to date only two case studies describing NUP214-ABL1 positive T-ALL patients treated 
with TKIs have been described, with mixed results. The first case showed no bone marrow response after being treated with $400 \mathrm{mg}$ imatinib daily for one-month post relapse, while the second was successfully treated with dasatinib monotherapy at time of diagnosis and attained a complete cytogenetic remission within three weeks. Here we present a patient with the NUP214-ABL1 fusion gene who has shown a favourable response to imatinib therapy post-relapse. The patient, a 41 year old male presenting with T-ALL, was found by conventional cytogenetics to have a karyotype of 46,XY,?inv(3)(p23q2?7)[20]. Fluorescence in situ hybridisation (FISH) with the LSI BCR-ABL dual fusion probe (Vysis) was performed, and an extra ABL1 signal was detected at $4 \mathrm{q} 21$ in addition to the ABL1 signals at 9q34. RT-PCR and SNP array analysis confirmed that the amplified fragment contained the NUP214-ABL1 fusion sequence. The patient initially received HyperCVAD induction chemotherapy, and attained a cytogenetic remission after two cycles of treatment. He received a sibling allograft 5 months after diagnosis, but relapsed 3 months later. The patient was subsequently treated with a combination of imatinib $(600 \mathrm{mg}$ daily), vincristine and prednisolone, and his response monitored by qualitative RT-PCR for the NUP214-ABL1 transcript. A quick remission was attained, and a decrease in NUP214-ABL1 transcript levels was detected over five months post-relapse.

\section{GLYCOGEN STORAGE DISEASE: TRANSITION TO ADULT SERVICES - PROCESSES AND CHALLENGES}

E. Clover', M. Netting', I. Chapman², D. Bratkovic ${ }^{3}$, D. Ketteridge ${ }^{3}$

${ }^{1}$ Department of Clinical Dietetics, Royal Adelaide Hospital, Adelaide, Australia ${ }^{2}$ Division of Medicine, University of Adelaide, Adelaide, Australia

${ }^{3}$ Metabolic Unit, Children, Youth and Women's Health Service, Adelaide, Australia

Background: Glycogen Storage Disease (GSD) is a disorder of carbohydrate metabolism due to absence or deficiency of one of the enzymes responsible for making or breaking down glycogen in the body. In severe forms of GSD, the risk of hypoglycaemia is treated with a diet of regular carbohydrate (CHO), uncooked cornstarch (UCCS), and if necessary overnight enteral feeds of a glucose polymer. Long-term complications include potentially malignant hepatic tumours and prospective liver transplantation. Aims: To review the medical and dietetic processes and challenges faced in the transition of the first two young adults from a paediatric to an adult metabolic service. Subjects: Two 18 year old men, one with GSD type Ia (glucose-6-phosphatase deficiency) and the other GSD type III (glycogen debrancher enzyme deficiency) were transitioned from a paediatric metabolic unit to an adult service in 2008. The GSD type Ia patient required a regular CHO diet, UCCS and overnight enteral feeds of a glucose polymer. The GSD type III patient required a regular $\mathrm{CHO}$ diet and UCCS before bed. Methods: Issues to be addressed in the adult service were:

- Patient issues: (1) education on the effects of alcohol consultation with hepatologists, (2) patient attendance to clinic, (3) treating the patient as an adult.

- System issues: (1) development of protocols for the Emergency Department (ED), (2) liaison with the formula room for emergency supplies of UCCS and glucose polymer, (3) introducing a new Home Enteral Nutrition program, (4) up-skilling an adult service dietitian, (5) appropriate alerts to the metabolic team during a patient admission.

Conclusions: Transitioning a patient with GSD from a paediatric service to an adult service provides challenges for the patient and the service. Early introduction to the new treating team to identify key patient and system issues prior to transfer is essential for smooth transition.

\section{HIGH-THROUGHPUT, POOLED SEQUENCING OF A PATIENT COHORT IDENTIFIES NOVEL GENES THAT CAUSE HUMAN MITOCHONDRIAL COMPLEX I DEFICIENCY}

A. G. Compton', S. E. Calvo 2,3,5, E. J. Tucker 1,6, D. M. Kirby', G. Crawford ${ }^{5}$, N. P. Burtt', M.A. Rivas ${ }^{2,5}$, C. Guiducci ${ }^{5}$, D. L. Bruno', O.A. Goldberger ${ }^{2,3}$, M. C. Redman ${ }^{5}$, E.Wiltshire ${ }^{7,8}$, C. J.Wilson', D.Altshuler ${ }^{2,4,5}$, S. B. Gabriel ${ }^{5}$, M. J. Daly ${ }^{2,5}$, V. K. Mootha ${ }^{2,3,5}$, D. R. Thorburn ${ }^{1,6}$

${ }^{1}$ Murdoch Childrens Research Institute and Victorian Clinical Genetics Services The Royal Children's Hospital, Melbourne, Australia

${ }^{2}$ Center for Human Genetic Research, Massachusetts General Hospital, Boston, United States

${ }^{3}$ Department of Systems Biology, Harvard Medical School, United States

${ }^{4}$ Department of Genetics, Harvard Medical School, United States

${ }^{5}$ Broad Institute of Harvard and MIT, Cambridge, MA, United States

${ }^{6}$ Department of Paediatrics, University of Melbourne, Melbourne, Australia

${ }^{7}$ Department of Paediatrics and Child Health, University of Otago, Wellington,

New Zealand

${ }^{8}$ Central Regional Genetics Service, Wellington, New Zealand

${ }^{9}$ National Metabolic Service, Starship Children's Hospital, Auckland, New Zealand

Complex I (NADH:ubiquinone oxidoreductase) is the first and largest complex in the mitochondrial respiratory chain. It contains 45 subunits, seven of which are encoded by mitochondrial DNA. Additional 'assembly factors' are required to correctly assemble subunits into a functional enzymatic complex. However, this process is still poorly defined. Deficiency of mitochondrial complex I is the most common cause of mitochondrial disease and can result from mutations in complex I subunit genes or assembly factors. However, we believe that mutations in the known genes account for the defect in $~ 50 \%$ of patients. Discovering the molecular basis of the remaining patients is challenging given their clinical heterogeneity and the large number of both mitochondrial and nuclear genes involved. We previously reported a number of potential new candidate genes involved in complex I biogenesis identified by phylogenetic profiling. Now we report results from pooled high-throughput sequencing of 103 genes encoding complex I subunits and proteins putatively involved in complex I biogenesis. Our cohort of 103 complex I deficient patients included 43 patients with known mutations, allowing us to demonstrate a sensitivity of $83 \%$ for detecting nuclear DNA mutations. We identified new genetic diagnoses in $22 \%$ of the remaining 60 patients, including mutations in known subunit and assembly factor genes. We also identified causative mutations in two novel complex I biogenesis genes, NUBPL and FOXREDI, and confirmed their pathogenicity by performing lentiviral correction studies in patient fibroblasts. Our study illustrates how high-throughput sequencing can reveal disease-causing mutations in individual patients and help uncover the molecular basis of complex I deficiency. However, this approach alone was unable to detect the causative mutations in $48 \%$ of our cohort. In a wider context, the study demonstrates that pooled high-throughput sequencing approaches can be an effective way to screen large numbers of candidate genes in patient cohorts.

PATTERNS OF REFERRALS AND TESTING FOR THE FMR1 GENE FOR THE LAST TEN YEARS IN VICTORIA

M. Cotter ${ }^{1,2}$,A.Archibald ${ }^{1,2}$, B. McClaren ${ }^{1,2}$,V. Petrou ${ }^{1,4}$, J. Cohen $^{3}$, D. Francis ${ }^{4}$ T. Burgess ${ }^{4}$, L. Hills ${ }^{4}$, R. Oertel ${ }^{4}$, H. Slater ${ }^{1,2,4}$, S. Metcalfe ${ }^{1,2}$

${ }^{\prime}$ Murdoch Childrens Research Institute, Royal Children's Hospital, Melbourne, Australia ${ }^{2}$ Department of Paediatrics, University of Melbourne, Australia

${ }^{3}$ Fragile X Alliance Inc. \& Centre for Developmental Disability Health Victoria, Department of General Practice, Monash University, Australia

${ }^{4}$ Victorian Clinical Genetics Service Pathology, Royal Children's Hospital, Melbourne, Australia

Molecular testing of the FMRI gene can identify individuals with fragile X syndrome (FXS) and other phenotypes related to the CGG expansion and methylation status. We present testing and referral patterns at Victorian Clinical Genetics Service Pathology from 2000 to 2009. Testing was performed on 19,112 individuals for the following clinical referrals: (1) developmental delay/global delay/intellectual disability/speech delay/learning difficulties, $72.7 \%$; (2) family history of FXS, 4\%; (3) autism/autism like spectrum, 17.6\%; (4) ovarian 
dysfunction, 1.6\%; neurological/FX tremor ataxia syndrome, $0.7 \%$; (5) epilepsy, $2.3 \%$; (6) other, $21.9 \%$; (7) not specified, $5.4 \%$. Referrals were made by clinical geneticists $(4.4 \%)$, GPs (3.4\%), paediatricians (49.5\%), obstetricians/gynaecologists (1.2\%), neurologists $(1.0 \%)$, pathologists $(19.5 \%)$, as well as other health professionals. Results from non-prenatal samples were: 187 (1.0\%) full mutations (FMs; 122 males, 64 females, 1 unknown); 316 (1.7\%) premutations (PMs; 84 males, 232 females); and 226 (1.2\%) grey zones (GZs; 82 males, 144 females). The age range (years) at testing was 0-90 overall. Median age at testing remained fairly constant over the 10year period: 5 years for males and 8-9 years for females. The majority of females with PMs were tested in their reproductive years. 128 tests were performed on CVS/amniocentesis samples with 39 FMs (30.1\%), 10 PMs (7.8\%) and $1 \mathrm{GZ}(0.8 \%)$ results found. The number of tests performed for clinical indications has been steadily increasing with 1607 tests in 2000 and 2379 in 2009. Before 2003, when the test became available on the Medicare Benefits Schedule, GZs were not reported. GZ results and PM results in females have significantly contributed to the number of test-positive reports. Following a positive result many individuals are not seen by the Victorian Clinical Genetics Service or the Fragile X Alliance Clinic. Further research is recommended to identify how families are being managed after receiving a test-positive result.

\section{'HE DIDN'T SAY THALASSAEMIA MIGHT COME UP' - EXPERIENCES AND ATTITUDES OF CARRIERS IN VICTORIA}

N. E. Cousens ${ }^{1,2}$, S.A. Metcalfe ${ }^{1,2}$, C. L. Gaff',2, M. B. Delatycki ${ }^{1,2,3}$

${ }^{\prime}$ Laboratory and Community Genetics, Murdoch Childrens Research Institute, Melbourne, Australia

${ }^{2}$ Department of Paediatrics, Faculty of Medicine, Dentistry and Health Sciences, The University of Melbourne, Australia

${ }^{3}$ Department of Clinical Genetics, Austin Health, Melbourne, Australia

Background: There is currently no universal beta-thalassemia carrier screening program in Victoria however antenatal carrier screening does occur through routine full blood examinations conducted early in a woman's pregnancy. This screening process has been highly successful at identifying at-risk couples as $78-100 \%$ of affected pregnancies between the years 2004-2008 were identified through prenatal diagnosis in Victoria. There is limited information about how women are screened for beta-thalassemia in Victoria, including whether they are notified that they are being screened and provide informed consent for the testing, as well as their attitudes towards this screening process. Aim: To explore the beta-thalassemia carrier screening process experienced by female carriers in Victoria and their attitudes towards this process. Method: Semistructured interviews were conducted with 26 female carriers of beta-thalassemia who are currently or have been pregnant within the last 12 months. Interviews were analyzed using thematic analysis. Results: There is great variability in the beta-thalassemia carrier screening process with different journeys experienced by each woman. Women were identified as carriers at different times of their lives, either before and/or during pregnancy. Most women were not informed that they were being screened for thalassemia until they were notified that they are a carrier. Although not providing consent was considered acceptable, with women happy for their doctors to decide what tests are conducted during their pregnancy, most women would have preferred to have been informed that they were being screened before being notified that they were a carrier. Conclusion: Most women in this study did not provide informed consent for thalassemia screening, but placed greater value on awareness of testing than choice or informed consent. These results suggest that routine screening as part of clinical care may be acceptable but further work to ascertain the views of a representative group, including carrier couples, is necessary.
DNA METHYLATION ANALYSIS IN MULTIPLE TISSUES FROM TWIN PAIRS AT BIRTH REVEALS BOTH GENETIC AND IN UTERO ENVIRONMENTAL/STOCHASTIC COMPONENTS TO HUMAN NEONATAL EPIGENOME

\section{ESTABLISHMENT}

J.M. Craig', M. Ollikainen', K. R. Smith², E. J. Joo', H. K. Ng', R.Andronikos', B. Novakovic', N. K. Abdul Aziz', J. C. Carlin², R. Morley', R. Saffery'

Developmental Epigenetics, Murdoch Childrens Research Institute, Melbourne, Australia

${ }^{2}$ CEBU, Murdoch Childrens Research Institute, Melbourne, Australia

We propose that the human epigenome is in constant flux from conception onwards, due to stochastic and environmental factors. This hypothesis is testable with monozygotic (MZ) twins and may explain differences in phenotype between MZ twin siblings. Genomic imprinting is an epigenetic phenomenon that inactivates one allele of a gene by methylation of $\mathrm{CpG}$ sites in a parent-of-origin-dependent manner. Imprints are established in the germline, are propagated stably during development, and may be sensitive to environmental factors. Differential methylation of imprinting control regions (ICRs) is involved in the regulation of the transcription of their target genes. There is evidence that MZ twins can have different epigenetic marks but it is unclear when such differences arise and how prevalent they are across different tissues. In order to address this, we examined methylation of four differentially methylated regions (associated with IGF2/H19 locus) in multiple birth tissues derived from 91 twin pairs: $56 \mathrm{MZ}$ and $35 \mathrm{DZ}$. Tissues included cord blood-derived mononuclear cells and granulocytes, human umbilical vein endothelial cells, buccal epithelial cells and placenta. This represents two embryonic germ layers and extraembryonic tissue. Considerable variation in methylation was observed between tissues in all individuals and between unrelated individuals in specific tissues. Most interestingly, methylation discordance was also present in individual tissues within all twin pairs with dizygotic (DZ) twins showing greater variability than monozygotic (MZ) pairs. These data highlight the contribution of both intrauterine environmental exposures and underlying genetic factors to the establishment of the neonatal epigenome, and confirm the intrauterine period as a sensitive time for the establishment of epigenetic variability in humans. This has implications for the effects of maternal environment on the development of the newborn epigenome and supports an epigenetic mechanism for the previously described phenomenon of 'fetal programming' of disease risk in humans.

\section{WHAT FACTORS INFLUENCE INDIVIDUALS WHEN DECIDING WHETHER OR NOT TO CONTACT A FAMILY CANCER CLINIC TO FIND OUT MORE ABOUT THEIR GENETIC TEST RESULTS AS A RESULT OF PARTICIPATING IN A POPULATION BASED RESEARCH STUDY?}

A. Crook ${ }^{* 1,2}$, L. Plunkett ${ }^{* 1,2,3}$, S. Wake ${ }^{2,4}$, M. Young

${ }^{\prime}$ Familial Cancer Clinic, Peter MacCallum Cancer Centre, Melbourne, Australia 'Department of Paediatrics, The University of Melbourne, Royal Children's Hospital Melbourne, Australia

${ }_{3}^{3}$ Genetics Education and Health Research, Murdoch Childrens Research Institute, Melbourne, Australia

${ }^{4}$ Genetic Health Services Victoria, Melbourne, Australia

* Joint first authors

The Australian Ovarian Cancer Study (AOCS) is an Australia-wide population based study which recruited women diagnosed with ovarian cancer between 2002 and 2006 and gathered both biospecimens and epidemiological data. The AOCS Genetic Profiling study is a side project, currently undertaking genetic testing to determine the prevalence of BRCA1/BRCA2 mutations in this cohort of women. If a mutation is identified, participants or their next of kin are notified in writing about the finding and availability of obtaining results at a familial cancer centre. Previous studies have generally shown a low uptake of people obtaining genetic test results in the 'research setting'. This study aims to increase understanding of the experiences and decision-making process of individuals offered 
genetic testing results obtained through a research project. AOCS research participants or next of kin are being interviewed regarding their decision making process of whether to follow up on the genetic information they are notified about through the study. Data is being collected through in depth telephone interviews, and analyzed using thematic analysis. To date, six individuals have been interviewed, of which only one was an original AOCS participant. The other five were next of kin, including one spouse, one son, two daughters and a daughter-in-law. For all the families approached, at least one family member has been interested in participating in this study. Results from the study will be presented, together with recommendations for genetic counseling practice in the research setting.

\section{IF AT FIRST YOU DON'T SUCCEED ... MAKING THE RIGHT DIAGNOSIS EVENTUALLY}

\section{K. Crowe', J. McGaughran ${ }^{2}$ \\ ${ }^{I}$ Nambour General Hospital, Genetic Health Queensland, Nambour, Australia \\ ${ }^{2}$ Royal Brisbane and Women's Hospital, Genetic Health Queensland, Brisbane, Australia}

In 2006, a patient presented to Genetic Health Queensland (GHQ) with a history of premenopausal breast cancer, bilateral dermoid ovarian cysts and multiple basal cell carcinomas (BCC). Her firstdegree relatives had histories of pre- and post-menopausal breast cancer, papillary thyroid cancer, bladder cancer and esophageal cancer. Based on her BRCAPRO score, testing for hereditary breast and ovarian cancer syndrome (BRCA1 \& BRCA2) was undertaken and no pathogenic mutations were identified. The differential diagnosis of Cowden syndrome (CS) was then considered given the family tumour spectrum; premenopausual breast cancer, thyroid cancer, and BCCs. However, macrocephaly was absent in the proband. Genetic testing of PTEN was undertaken and no pathogenic mutation was found. In 2010, the proband represented to GHQ with a sebaceous adenoma that demonstrated immunohistolological loss of $\mathrm{MSH2}$, one of five mismatch repair (MMR) genes responsible for Lynch syndrome. Genetic testing consequently found the proband to be a carrier a truncating $\mathrm{MSH} 2$ mutation. Lynch syndrome (HNPCC) is a dominantly inherited disorder characterized by the development of a variety of cancers, predominantly colorectal and endometrial malignancies. However no commonly associated tumours have been reported in this family. This case outlines some cancer genetic counseling issues that evolved and the emerging implications for family members with the culmination of the diagnosis Lynch syndrome specifically Muir-Torre syndrome.

\section{ATTITUDES TOWARD POPULATION-BASED CARRIER SCREENING FOR CYSTIC FIBROSIS (CF) BY CF HEALTHCARE PROFESSIONALS}

\section{F. Cunningham', S. Lewis ${ }^{2}$, J. Glazner', J. Massie ${ }^{1,2,3}$}

Paediatrics, University of Melbourne, Australia

${ }^{2}$ Public Health Genetics, Murdoch Childrens Research Institute, Melbourne, Australia Respiratory Medicine, Royal Children's Hospital, Melbourne, Australia

Carrier screening for cystic fibrosis (CF) is available but there is not a national population-based screening program in Australia. Knowledge of Australian CF healthcare professionals' attitudes towards carrier screening could provide useful information to implement such a program. The aim of this study was to investigate the attitudes of CF respiratory physicians and CF clinic coordinators in Australia towards population-based carrier screening for CF. A purpose-designed questionnaire assessing knowledge and attitudes towards $\mathrm{CF}$ carrier screening was distributed to 110 respiratory physicians and $30 \mathrm{CF}$ clinic coordinators throughout Australia. Seventy-five responded, 55 physicians $(49.5 \%)$ and 20 coordinators (67\%). Sixty-four respondents $(85 \%)$ rated raising a child with CF as difficult/very difficult, $63(84 \%)$ rated the shortened life span as a significant concern and $64(85 \%)$ the daily treatment regimen as a significant concern. Disadvantages of screening were perceived anxiety amongst carriers $(n=65,87 \%)$ and discrimination of carriers $(n=42,56 \%)$. Respondents rated the following barriers as most important: limitations of predicting clinical outcomes $(n=47,65 \%)$ and insufficient resources for providers $(n=45,61 \%)$. Fifty-four $(76 \%)$ of respondents believed they had a role in the development of a CF carrier-screening program. Forty-two (56\%) respondents were in favour of population-based carrier screening for CF. Those who supported screening rated the chance of being a carrier, reassurance to test negative people and the daily CF treatment as important factors. Opposition to screening was associated with identifying discrimination against carriers as a disadvantage, and limitations of predicting clinical outcomes as important barriers to screening. A small majority of CF healthcare professionals in Australia are in favour of population-based carrier screening for CF. Barriers to implementation include dealing with carrier anxiety, difficulty predicting clinical outcome from genotype and counseling resources. $\mathrm{CF}$ healthcare professionals have raised useful concerns regarding the development of a CF carrier-screening program in Australia.

\section{TAY SACHS POPULATION CARRIER SCREENING IN THE ASHKENAZI JEWISH COMMUNITY: AN EVALUATION OF THE TSD CARRIER SCREENING PROGRAM IN MELBOURNE HIGH SCHOOLS}

H. Curd', I. Macciocca ${ }^{4}$, M. Sahhar ${ }^{4}$, S. Lewis ${ }^{2}$, M. Delatycki ${ }^{3,5}$

${ }^{\prime}$ Department of Paediatrics, University of Melbourne, Australia

${ }^{2}$ Public Health Genetics, Murdoch Children's Research Institute, Melbourne, Australia

${ }^{3}$ Bruce Lefroy Centre for Genetic Health Research, Murdoch Children's Research Institute, Melbourne, Australia

${ }^{4}$ Genetic Health Services Victoria, Melbourne, Australia

${ }^{5}$ Clinical Genetics, Austin Health, Melbourne, Australia

The Melbourne school-based TSD carrier-screening program has not undergone a long term evaluation since its implementation in 1997. The aim of the study was to assess the outcomes of the Melbourne TSD carrier screening program among those who had testing more than 5 years ago, to assess the long term impact of carrier testing during high school, including psychological outcomes, knowledge of $\mathrm{TSD}$, memory of test result, use of test result and retrospective opinion about the screening program. A questionnaire was used for data collection and it consisted of validated scales and purposively designed questions. All carriers who were identified between 1999 and 2005 were sent an invitation to participate in the study $(n=69)$ and for each carrier identified, two non-carriers who were also screened in the program were randomly selected and sent an invitation to participate, after matching for gender, school and year of screening $(n=138)$. Of these, 19 were returned addressee unknown, and 4 responded that they were overseas and could not complete the questionnaire. Of the remaining 184 questionnaires (62 carriers and 122 non carriers), 54 were completed and returned, giving a total response of $29.3 \%$. Most participants (82\%) still had good knowledge of TSD and there was no evidence of a difference in knowledge between carriers and non-carriers. Most participants were happy with the timing and setting of screening and thought that education and screening for TSD should be offered during high school. There was no evidence of any long-term negative psychological impact of the screening program, as measured by the State Trait Anxiety scale, Impact of Event scale and Decision Regret Scale. This evaluation study supports the continued use of the TSD screening program in Melbourne high schools.

CAUSES OF EPIGENETIC MUTATIONS IN A CHILDHOOD CANCER PRE-DISPOSITION SYNDROME

\section{Dagar ${ }^{1,2}$, E. Algar $^{1,2}$}

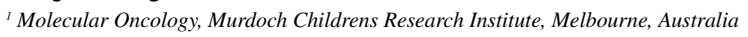
${ }^{2}$ University of Melbourne, Australia

Beckwith Wiedemann Syndrome (BWS) is an imprinting disorder characterized by overgrowth and an increased risk of cancer. It is one of the most common imprinting disorders with a prevalence of approximately 1 in 13,700 live births, but this is thought to be an underestimate because of the presence of milder, undiagnosed cases of BWS. The known causes of BWS are varied and encompass epigenetic mutations including loss of methylation (LOM) at 
$K C N Q 1 O T 1$, gain of methylation at $H 19$ along with UPD of chromosome 11p15.5, CDKNIC mutations and a minority group with cytogenetic abnormalities affecting $11 \mathrm{p} 15$. The maintenance of methylation at imprinted centres post-fertilization is performed by DNMT1 that is the principal methyltransferase in vertebrates. We have hypothesized that as yet unidentified variations or mutations in the DNMT1 gene could result in failure of maintenance of methylation at the KCNQ1OT1 imprinting centre. Although we have not identified any pathogenic mutations in DNMT1 to date, a heterozygous substitution NM_001379.2:c.406 C>T, p.Arg136Cys which is incompatible with DNMT1 protein folding was found in one BWS case. We have also screened genes in the one-carbon folate pathway including MTHFR, MTR or MTRR in 49 cases of BWS with isolated LOM. We have identified an increased prevalence of the MTHFR C677T polymorphism in these cases. In the cohort the $\mathrm{T}$ allele frequency was $36 \%$ (35/98) compared to a control frequency of $26 \%$ $(51 / 200)(p=.077)$. Other SNPs in MTHFR (A1298C, G1793A), MTR (A2756G) and MTRR (A66G) that were analyzed in this study were equally represented in BWS and control cases.

\section{MODELING OF FOOD GROUPS TO MEET RECOMMENDED DIETARY REQUIREMENTS ON VARYING REDUCED PROTEIN INTAKES IN FOUR AGE GROUPS}

\author{
H.Teoh', B. Dennison', P.Watson'2, S. Thompson ${ }^{2}$ \\ ${ }^{\prime}$ Nutrition and Dietetics, The University of Newcastle, Australia \\ ${ }^{2}$ Nutrition and Dietetics/Western Sydney Genetics Programme, The Children's Hospital \\ at Westmead, Australia
}

Children with disorders of protein metabolism such as hyperphenylalaninemia, urea cycle disorders and some organic acidaemias, who are on restricted food protein intakes and who do not take a synthetic nutritional supplement are at risk of micronutrient deficiencies. Using the FOODWORKS food analysis program we analyzed theoretical diets with varying levels of protein restriction for children aged 9 and 18 months, and 3 and 8 years. For each age group we analyzed 4 different degrees of protein restriction and compared the results with Nutrient Reference Values (NRVs) for Australia and New Zealand, 2005. Level 1 excluded protein from all animal sources; level 2 excluded meat, fish, chicken and egg, and included small amounts of dairy products; level 3 included small amounts of legumes and/or egg, and some dairy products; level 4 included small amounts of animal protein, and dairy products. There was no restriction of protein from grains, fruits or vegetables at any level. Calcium, iron, zinc, vitamin B12 and riboflavin were shown to be the most vulnerable nutrients in levels 1 to 3 protein-restricted diets. Children with a greater protein tolerance however, still need to make careful food choices in order to meet their nutritional needs. A resource for patients was developed to provide food options to minimize the likelihood of deficiency.

\section{SHARING THE NEWS - UPTAKE OF PREDICTIVE GENETIC TESTING WITHIN FAMILIES WITH A FAMILIAL COLORECTAL CANCER SYNDROME}

\section{S. Doak', M. Clancey², J. Bjorkman ${ }^{3}$, L. Hunt ${ }^{2}$ \\ ${ }^{\prime}$ Genetic Health Queensland, Gold Coast, Australia \\ ${ }^{2}$ Genetic Health Queensland, Brisbane, Australia \\ ${ }^{3}$ Pathology Queensland, Brisbane, Australia}

Genetic Health Queensland (GHQ) offers testing for familial colorectal cancer syndromes such as Lynch syndrome, Familial Adenomatous Polyposis (FAP) and MYH-associated polyposis to individuals who meet clinical criteria. Upon identification of a mutation associated with a familial colorectal cancer syndrome, the importance of sharing this information within the family is discussed with the proband and various communication strategies are explored. It is not within the practice of GHQ to contact family members directly to advise them of their potential increased risk of developing cancer and the availability of predictive genetic testing. Instead, this is the responsibility of the proband and is often facilitated by providing a generic letter ('family letter') that the proband can share with their family members. In order to evaluate the effectiveness of this process, we performed a retrospective study of families offered genetic testing for a familial colorectal cancer syndrome in which a mutation was identified. The number of family members subsequently referred to GHQ for predictive testing was then assessed. The information analyzed and discussed includes the degree of relatedness to the proband, the length of time between informing the proband of their result and the referral of other family members and whether the 'family letter' was utilized. Consideration was given to factors such as family members living interstate or overseas and communication barriers within the family. The limitations of the 'family letter' and this review are also presented.

\section{THE ROLE OF SIGMAR1 IN SINGLE-GENE AND COMPLEX} NEURODEGENERATIVE TRAITS

C. Dobson-Stone ${ }^{1,2}$, A.A. Luty', O. Piguet ${ }^{1,2}$, G.A. Broe ${ }^{1,2}$, G. M. Halliday,

P. R. Schofield ${ }^{1,2}$, J. B.J. Kwok ${ }^{1,2}$

${ }^{2}$ Neuroscience Research Australia, Sydney, Australia

${ }^{2}$ School of Medical Sciences, University of New South Wales, Australia

Neurodegeneration genes can harbour rare mutations that lead to single-gene diseases and common polymorphisms that increase susceptibility to more complex disorders. An example is the microtubule-associated protein Tau gene (MAPT), in which missense mutations cause early-onset familial forms of frontotemporal dementia (FTD), while the common MAPT H1 single-nucleotide polymorphisms (SNPs) increase susceptibility to late-onset idiopathic FTD and Alzheimer's disease (AD). We have identified a mutation in the Sigma nonopioid intracellular receptor 1 gene (SIGMAR1) in a large, multi-generational family with dominant inheritance of FTD and/or motor neuron disease. The mutation is in the 3' untranslated region (3'UTR) of the gene and significantly alters gene expression in vitro and in patient lymphocyte and brain samples. Sigma-1 is a brain receptor that binds several classes of psychotropic drugs and neurosteroids and that may act as an intracellular protein chaperone. Modulation of Sigma-1 activity by overexpression, RNA interference knockdown or synthetic ligands leads to intracellular redistribution of TDP-43 and FUS, two proteins believed to play a major pathological role in FTD and MND. Polymorphisms in SIGMARI have also been associated with lateonset AD. We examined SNPs either within or in linkage disequilibrium (LD) with SIGMARI in two community cohorts with extensive longitudinal phenotype data. In the Sydney Older Persons Study (SOPS) cohort, we detected a significant association between a non-synonymous SIGMARI SNP and dementia in males. In the Framingham Heart Study SNP Health Association Research cohort, we detected a significant association between a four-SNP haplotype of the SIGMARI LD block and mini-mental state examination score. Our results suggest that SIGMARI may play a pathological role in neurodegenerative diseases, either via rare mutations leading to a significant alteration of SIGMARI expression, or by more common polymorphisms that may lead to a more subtle change in SIGMARI expression or function.

\section{QUANTIFICATION OF MITOCHONDRIAL MUTATION MUTANT LOAD USING HIGH RESOLUTION MELT ANALYSIS}

\section{Du Sart, S. Pantaleo, B. Chong}

Molecular Genetics, VCGSPathology, Murdoch Childrens Research Institute, Melbourne, Australia

Mitochondria are essential components of every cell, carrying out aerobic metabolism in order to generate energy. They have their own genome, a $16.6 \mathrm{~kb}$ circular molecule encoding 37 genes that produce 13 proteins incorporated into the respiratory chain. There are 2-10 copies of mitochondrial DNA (mtDNA) in each mitochondrion and many mitochondria in every cell, therefore many copies of mtDNA in each cell. mtDNA alterations may be present in some mtDNA molecules and not others (heteroplasmy). This creates a threshold effect, whereby a certain number of mtDNA molecules must carry the alteration in order to have a deleterious effect on the tissue. 
Quantification of mutant load (the amount of mtDNA molecules possessing a mutation) is a challenge for the laboratory. Past techniques involved last cycle addition of radioactive phosphorus into PCRs and subsequent phosphoimaging, which enabled the comparison and quantification of the amount of normal to abnormal mtDNA molecules. Currently in the lab we are working on using High Resolution Melt (HRM) to assist in the quantification of mitochondrial mutation mutant load. HRM is a technique that has many applications, including mutation discovery and SNP genotyping. It is both cost and time effective. HRM is based on characterizing DNA samples according to their melting behavior. Hence a DNA sample with a single nucleotide change will produce a DNA dissociation curve of a different pattern to that of the wild-type.

\section{THE LONG AND WINDING ROAD: FACTOR V LEIDEN} AND ADVERSE PREGNANCY OUTCOME.

\section{T. Dudding \\ Hunter Genetics, Hunter Area Health Service, University of Newcastle, Australia}

Are inherited thrombophilias associated with an increased risk of adverse pregnancy outcome? And how should clinicians interpret conflicting recommendations and make evidence-based decisions. Numerous case control studies, cohort studies and meta-analyses have been conducted to establish a link between adverse pregnancy outcomes and factor V Leiden or prothrombin 20210 G/A gene variant. Guidelines addressing the screening and management of pregnant women with thrombophilia have been published in America and Europe, including the United Kingdom, but inconsistencies between them make it problematic for clinicians looking to make evidence-based, practice decisions. There are a number of possible explanations for these inconsistencies. They include the lack of a homogeneous grading system, conflicting 'expert opinion' and differences in the interpretation of evidence. This presentation is a collation of published case control and cohort meta-analyses exploring the possible association between factor $\mathrm{V}$ Leiden or prothrombin 20210 G/A gene variant with adverse pregnancy outcomes. It includes outstanding clinical questions, challenges faced by researchers and important areas for future research.

\section{A NOVEL MUTATION IN THE ERYTHROPOIETIN RECEPTOR GENE IS ASSOCIATED WITH PRIMARY FAMILIAL AND CONGENITAL POLYCYTHEMIA (PFCP)}

D. J. Fairbairn', K. O'Rourke², K. Jackson², K. Morris'², S. Tey², G.A. Kennedy ${ }^{2}$

${ }^{\prime}$ Department of Haematology, Molecular Genetics Laboratory, Pathology Queensland, Brisbane, Australia

${ }^{2}$ Department of Haematology, Royal Brisbane and Women's Hospital, Brisbane, Australia

Primary familial and congenital polycythemia (PFCP), or familial erythrocytosis-1, is an autosomal dominant disorder characterized by increased hemoglobin, red cell mass, hypersensitivity of erythroid progenitors to erythropoietin (EPO) and low serum levels of EPO. Erythropoietin controls the proliferation, differentiation and survival of erythroid progenitors. Mutations in the gene encoding the erythropoietin receptor gene $(E P O R)$ have been associated with PFCP. A 15-year-old male was referred for investigation of polycythemia $(\mathrm{Hb}$ $189 \mathrm{gm} / \mathrm{L}$; Hct 0.58 ). FBC parameters were normal, and clinical examination unremarkable. Subsequent testing for the JAK2:p.Val617Phe mutation was negative, bone marrow examination unremarkable, but serum erythropoietin level was only 1 $\mathrm{mIU} / \mathrm{mL}$ (4-32). The patient had a strong family history of polycythemia with an autosomal dominant mode of inheritance affecting at least 4 generations of family members. An increased rate of premature cerebrovascular disease was apparent in affected individuals. PCR and bidirectional sequence analysis was employed to screen for mutations in the EPOR gene. A novel heterozygous frame shift mutation EPOR:c.1234delT (p.Ser412ArgfsX41) residing in exon eight of EPOR was subsequently identified. Similar to other EPOR mutations described to date, our identified mutation leads to a truncation of the cytoplasmic domain of the receptor, with resultant increased sensitivity of erythroid progenitors to EPO via the loss of several pathways involved in negative regulation. This lack of downregulation of signaling via the EPOR results in continued stimulation of erythroid proliferation in the setting in low concentrations of EPO and resultant erythrocytosis. Clinically, this mutation appears to be associated with increased risk of premature cerebrovascular disease in affected individuals.

\section{GENETIC COUNSELING IN FAMILIES WITH MULTIPLE AFFECTED INDIVIDUALS OF DISORDER OF SEX DEVELOPMENT: THE IMPACT OF CULTURE IN INDONESIA}

S. M.H. Faradz ${ }^{2}$,A. Ediati', B.A. Setyawati ${ }^{3}$,A. Juniarto', S. ${ }^{4}$, H. ${ }^{5}$, S. L.S. Drop ${ }^{1,5}$

${ }^{\prime}$ Human Genetics, Diponegoro University, Indonesia

${ }^{2}$ Faculty of Psychology, Diponegoro University, Indonesia

${ }^{3}$ Master program of Genetic Counseling, Diponegoro University, Indonesia

${ }^{4}$ Dept of Paediatrics, Murdoch Children Research Institute, University of Melbourne, Australia

${ }^{5}$ Pediatric Endocrinology, Erasmus Medical Center, Rotterdam, Netherlands

Genetic counseling is not yet common in Indonesia, but the awareness on understanding genetic disease and its management is slowly emerging. Disorder of Sex Development (DSD) brings challenging management in particular if identified at later age, creating quite complexities of problems within the families. Four families with different variants of multiple affected DSD were observed, namely siblings with 46,XY DSD gonadal dysgenesis who raised differently (as male and female); siblings with $46, \mathrm{XY}$ familial gonadal dysgenesis DAX1 duplication who raised as females, cousins of 46,XY DSD female with Complete Androgen Insensitivity Syndrome (CAIS), and 46,XX males with Congenital Adrenal Hyperplasia $(\mathrm{CAH})$. Their diagnosis was established by recent consensus of DSD by means of physical, hormonal, cytogenetics and molecular genetics examination. The complexities of problems hindered the process of family decision-making, especially when gender change issue was considered. Their decision for gender assignment and genital correction was influenced by their culture, belief and understanding on genetic diseases. Culture seems influenced family system or pattern of communication among these family members. Emotional problems, rumor, discrimination, isolation, devaluation from friends and community because of their DSD condition put them into struggle in daily lives. The compounding problems had challenged our understanding as genetic counselor towards local values and beliefs regarding marriage and family, having children and DSD condition, as well as religious view to help them work out their dilemmas. Education for medical professionals, patients, family members and community to break down prejudice against people having DSD should be promoted to improve their quality of life.

\section{CFTR MUTATION SCREENING IN AN INFERTILE POPULATION}

\section{P. Field, N. Martin}

QFG Genetics, Queensland Fertility Group, Brisbane, Australia

Cystic Fibrosis is the most common deleterious recessive single gene disease in non-Hispanic Caucasians. Mutations within the CFTR gene produce a variable phenotype including pulmonary disease, pancreatic insufficiency, meconium ileus and also infertility. The clinical phenotype is variable and depends on the combination of mutations present in the CFTR gene. The most severe phenotype is homozygous c.1521_1523delCTT (F508del) affecting the lungs, pancreas and intestinal tract and causing infertility, contrasting with the mild affects of c.350G $>$ A $(\mathrm{R} 117 \mathrm{H})$ causing only absence of the vas deferens in some male patients.

A population of 5000 patients presenting with Infertility at Queensland Fertility Group was screened for 30 CFTR gene mutations (Abbott/Celera Diagnostics Cystic Fibrosis v3 Genotyping Assay) using a two-step testing model. An overall carrier rate of 1 in 22.4 or $4.46 \%$ was identified in the patient population presenting with infertility $(3.89 \%$ to $5.03 \% \pm 95 \% \mathrm{CI})$. This compares to a 1 in 30 or $3.27 \%$ carrier rate $(2.66 \%$ to $3.87 \% \pm 95 \% \mathrm{CI})$ identified in an antenatal clinic setting in Victoria, reported in 2009. Overall, 15 different 
mutations were detected using a PCR/OLA assay based on ACMG/ACOG guidelines, with c.350G $>$ A (R117H) the second most frequent mutation identified.

The preconception screening program at Queensland Fertility Group not only enabled the identification of carrier patients but also provided the opportunity for patients to make informed decisions about their future reproductive treatment options prior to beginning treatment cycles.

With the HGSA's position statement advocating all couples be made aware of CFTR mutation screening, along with the indentified increased carrier rate in the infertile population, preconception screening should be encouraged for all infertile couples. It is our opinion that the infertile population be recognized as a sub-group for preconception screening and should include c.350G $>$ A (R117H) because of its high association with infertility.

\section{REGIONAL DIFFERENCES IN THE DIAGNOSIS RATES OF NIEMANN PICK C IN AUSTRALIA}

\section{J. M. Fletcher, M. Fietz \\ National Referral Laboratory, Genetics and Molecular Pathology, SA Pathology, Adelaide, Australia}

Introduction: Niemann Pick C (NPC) is a rare, difficult to diagnose, lipid storage disorder characterized by progressive neurodegeneration. A single laboratory is responsible for all Australian NPC diagnoses. Methods: Demographic data, including state of residence and date of diagnosis, for patients with a positive NPC diagnosis were determined from laboratory records. Age was available for all patients diagnosed after 1996. Incidence rates were calculated using population data from the Australian Bureau of Statistics. Data analysis was performed using Chi square statistic. Results: Sixty-one Australian patients have been diagnosed with NPC at the National Referral Laboratory, 34 since 1996 . Over the past 14 years, the annual incidence was 1 per 7.3 million population. Thirty-eight percent of the patients came from Victoria, a state with $25 \%$ of Australia's residents. Twelve of these were presumed to be infantile, 12 juvenile and 14 adult onset. Discussion: A disproportionately large number of NPC diagnosed patients came from a single state, with less than expected from the largest state. Conclusion: Clinician acumen and knowledge of NPC is the most likely explanation for the regional difference in diagnosis rate.

\section{WHEN FEARS COLLIDE: A CASE REPORT EXPLORING THE CANCER ANXIETY OF A BALANCED TRANSLOCATION CARRIER}

L. Fowles, A. Hattam, R. D. Susman

Genetic Health Queensland, Royal Brisbane and Women's Hospital, Brisbane, Australia

An important part of our role as genetic counsellors is assessing the relevant health risks faced by our clients. In the field of cancer genetics, clients have a preconceived view of their cancer risk. This is often formulated in part by their personal experiences. Part of our role is to explore our client's perception and try to address their beliefs so that their perceived cancer risk becomes more closely aligned with an objective assessment. There are times however when there may be some valid grounding to what initially appears to be an unfounded cancer fear. In this case, a patient was referred to the genetics service due to her extreme anxiety about her breast cancer risk. Formal assessment of her family history using the NBOCC guidelines placed her in the moderately increased risk category. However her anxiety was exacerbated by the knowledge that she carried two balanced chromosomal rearrangements. In most cases, a balanced translocation does not alter a person's cancer risk. An increased cancer risk could arise if a breakpoint affected the expression of a cancer predisposition gene, either through direct disruption of the gene sequence or disruption of a regulatory region. On review of our patient's karyotype, one of the breakpoints was found to be in the same chromosomal region as the $B R C A 2$ gene. Here we review our approach to assessing the $B R C A 2$ gene and addressing our client's anxiety.
THE NDUFS4/- MOUSE: A MODEL TO STUDY THE NEUROPATHOGENESIS OF MITOCHONDRIAL DISEASE

A. E. Frazier', J. Komen',A. Laskowski', M. McKenzie'2, M. Bird', M.T. Ryan², D. Thorburn'

'Mitochondrial Research, Murdoch Childrens Research Institute, Melbourne, Australia ${ }^{2}$ Biochemistry, La Trobe University, Australia

Disorders of mitochondrial oxidative phosphorylation (OXPHOS) comprise one of the most common groups of inborn errors of metabolism, causing a range of symptoms that potentially affect any or all organs, particularly the brain, heart and muscle. Severe neurological defects are common and often result in disability and death. Most OXPHOS disorders result from defects in Complex I (NADHubiquinone oxidoreductase). Composed of 45 subunits, 7 of which are mtDNA encoded, mutations resulting in isolated Complex I deficiency have been found in genes encoding subunits and assembly factors. The most common Complex I deficiencies, Leigh Syndrome (LS) and Lethal Infantile Mitochondrial Disease (LIMD), both have a prominent neurological component. Our understanding of the neuropathogenesis of mitochondrial disease has been limited by the lack of suitable model systems and tissues for study. However, we now have one of the first mouse knockout models of Complex I deficiency, the $\mathrm{Ndufs}_{s} 4^{-/}$mice, which display a severe progressive neuromuscular phenotype. Representing a model of Leigh Syndrome, these mice have reduced Complex I activity and 'crippled' Complex I assembly in all tissues studied, similar to patients. We have established primary neuronal cell cultures from these mice and begun to assess parameters that are implicated in the neuropathology of OXPHOS disorders, including ATP generation, mitochondrial membrane potential and $\mathrm{Ca}^{2+}$ dynamics. We have found that neurons from the Ndufs $4^{-1}$ mice display defects in ATP generation. In comparison, embryonic fibroblasts from these same mice generate ATP in comparable amounts to wildtype mice. This mirrors similar studies we have performed using skin fibroblasts from patients with Ndufs4 mutations, whereby no defects in ATP generation were observed. This observation has implications for our understanding of the neuropathogenesis of mitochondrial disease, and underscores the relevance of studying appropriate tissues in defining the pathways of cellular damage resulting from Complex I deficiency.

\section{NEW DIRECTIONS: ESTABLISHING A MULTIDISCIPLINARY} CLINIC FOR THE INHERITED PORPHYRIAS

L. Freeman', J. Pinner', V. Poulos², J. Bojovic', S. Mead', R. Trent', P. Stewart ${ }^{2}$

${ }^{\prime}$ Department of Molecular and Clinical Genetics, Royal Prince Alfred Hospital, Sydney, Australia

${ }^{2}$ Department of Clinical Biochemistry, Royal Prince Alfred Hospital, Sydney, Australia

Seven different porphyrias form a group of inherited metabolic disorders, each resulting from a partial deficiency of a specific enzyme in the hem biosynthesis pathway. Porphyrias are rare and sometimes misdiagnosed, because symptoms and signs may mimic other diseases. Clinically, the porphyrias may present as acute painful attacks with neurovisceral symptoms and acute or chronic skin symptoms. Porphyrias are mainly inherited in an autosomal dominant manner with incomplete penetrance. Variable expression in carriers suggests a role for modifying genes and environmental factors in the phenotype. Early diagnosis and information about precipitating factors can diminish mortality and prevent subsequent attacks among patients with acute porphyrias. The Departments of Clinical Biochemistry and Molecular \& Clinical Genetics at RPA Hospital are collaborating to establish a multidisciplinary clinic to offer a comprehensive service for individuals and their families with inherited porphyrias. Previously, testing was based on biochemical analysis of symptomatic individuals. This testing was not useful for individuals never experiencing symptoms. Genetic testing has allowed the identification of individuals with a pre-disposition to Porphyria. Mutation-positive individuals can be counseled about appropriate management to avoid or minimise disease complications whilst mutation-negative family members can be freed from unnecessary vigilance and psychological worry. There are a number of difficulties 
in undertaking the testing. Genetic testing is costly and genetic counseling of children and young adults in porphyria families can be challenging. Particularly when to offer genetic testing, given the natural history of the conditions and the potential exposure to triggers during adolescence. This presentation discusses these issues and the experience of the recently established RPA Hospital Inherited Porphyria Clinic.

\section{WHAT DETERMINES THE LEVEL OF SATISFACTION FOR CLIENTS OF THE OUTREACH GENETIC COUNSELING SERVICES IN THE MID-NORTH COAST, NSW?}

K. L. Frumar', B. K. Hopper ${ }^{2}$, C. J. Sorensen', J. Stofmeel ${ }^{3}$, G. Turner ${ }^{4}$

${ }^{I}$ Hastings-Macleay Network, North Coast Area Health Service, Port Macquarie, Australia

${ }^{2}$ Taree Community Health, Hunter New England Area Health Service, Taree, Australia ${ }^{3}$ Coffs Harbour Health Campus, North Coast Area Health Service, Coffs Harbour, Australia

${ }^{4}$ Mid North Coast Genetic Service, NSW, Australia

Genetic counsellors from three outreach genetic counseling services in the Mid-North Coast of NSW undertook to assess which aspects of the service determined the level of satisfaction for clients. The aim was to attempt to ascertain whether genetic counseling process attributes of access, follow-up, tailored case management and quality of client-clinician relationship as identified by McAlister at el. (2008) or the need for certainty of diagnosis as identified by Skirton et al, $(2005,2006)$ and Hernandez et al. (2006) determined or influenced the value or satisfaction for the client. A postal survey was sent to 338 clients of the service who had utilized the service during the period 1 January 2008-31 December 2009. Each survey, while maintaining anonymity, was coded to indicate whether the recipient had received a diagnosis, either new or previously, or whether no diagnosis had yet been established. Participants were asked to indicate their primary goal for seeking Genetic Counseling and their satisfaction with various aspects of the service. 82 surveys were returned (24\%). $40 \%$ of the respondents indicated that their primary goal was to find a diagnosis. Most respondents (78\%) reported high levels of satisfaction irrespective of their primary goal, and even when that goal was not met. $22 \%$ reported dissatisfaction with some aspect of the service. Of these, $50 \%$ had not obtained a diagnosis. Areas of dissatisfaction included failure to establish a diagnosis; a perceived lack of follow-up; lack of access to the service due to distance or unavailability of a GC; or a feeling of inadequate time during consultations.

\section{A CASE OF MOSAIC TRISOMY 22 WITH LITERATURE REVIEW}

\section{A. Galang, L. Carey \\ Genetics, Sydney IVF, Sydney, Australia}

We report on a 35-year-old female (G1P0) following a combined high risk nuchal translucency and first trimester biochemical screen $(\mathrm{FBHCG}=4.79 \mathrm{MoM}, \mathrm{PAPPA}=0.41 \mathrm{MoM}, \mathrm{NT}=2.18 \mathrm{~mm})$ at 11 weeks 4 days gestation. Karotyping was performed on CVS and showed a trisomy 22 female karyotype $-47, \mathrm{XX},+22$ in all cells examined. A follow-up ultrasound at 13 weeks detected intrauterine growth retardation, a single umbilical artery and suspected cardiac abnormalities, suggesting that the fetal karyotype was likely to be abnormal and not confined to the placenta. Follow up amniotic fluid karyotyping revealed a level III mosaic trisomy 22 karyotype $47, \mathrm{XX}+22[29] / 46, \mathrm{XX}[26]$. The patient continued with the pregnancy and further investigations included a fetal echocardiogram that showed a ventricular septal defect and pulmonary stenosis. A fetal MRI did not show any structural abnormalities however suggested the possibility of a neuronal migration disorder. The pregnancy is ongoing at the time of printing. Non-mosaic trisomy 22 although uncommon in live births, is the second most frequent aneuploidy identified in spontaneous miscarriage. Mosaic trisomy 22 whilst a rare finding, may be compatible with live birth and prolonged survival. Postnatally, mosaic trisomy 22 has varied clinical outcomes that may include growth retardation, mental retardation, and cardiac, ear and limb malformations. We review this case and other cases in current literature regarding the variable clinical presentations and difficulties in genetic counseling.

\section{RELATIVES' EXPERIENCES OF COMMUNICATING GENETIC INFORMATION FOLLOWING THE UNEXPECTED DEATH OF A FAMILY MEMBER DUE TO AN INHERITED CARDIAC CONDITION}

\section{Gallacher ${ }^{1,2}$, S. Metcalfe ${ }^{1,2}$, N. Morgan ${ }^{3}$, I. Macciocca ${ }^{4}$, J. Hodgson ${ }^{1,2}$ \\ ${ }^{I}$ Genetics Education and Health Research, Murdoch Childrens Research Institute, Melbourne, Australia \\ ${ }^{2}$ Department of Paediatrics, The University of Melbourne, Australia \\ ${ }^{3}$ Victorian Institute of Forensic Medicine, Melbourne, Australia \\ ${ }^{4}$ Genetic Health Services Victoria, Melbourne, Australia}

The sudden death of a relative is a traumatic event that is likely to disrupt the equilibrium in a family. The genetic implications of sudden death, particularly when it raises the possibility of a cardiac genetic condition in the family, are increasingly being recognized and clinical screening of relatives is being recommended with the autopsy findings. In Victoria, the senior next of kin of the deceased is usually contacted and informed of these familial implications. However, the communication of genetic information in families can often be a difficult process, even when a death has not occurred. This study used in-depth interviews to explore the experiences of families whose relative had died suddenly and where there was a possibility of genetic implications. Participants discussed the processes of hearing about the death, the legal and medical findings, how familial health implications are shared with surviving relatives, and available supports. Twelve interviews have been conducted with partners, parents and siblings of sudden death victims, where the death occurred between two and six years ago. The interviews were analyzed using thematic analysis. Participants described feelings of shock and having a blurred recollection of the time immediately following the death. They expressed difficulty in coping with uncertainty, when the cause of death was unascertained and when a diagnosis was not forthcoming. The majority identified helpful support measures. Occasionally instances in which support was inadequate were described. Participants recognized the importance of communicating genetic information at this time but also identified a burden associated with it. These emerging themes provide valuable insight into families' experiences and have implications for service providers in this field.

\section{DIRECT-TO-CONSUMER GENETIC TESTING}

\section{Gardner'}

${ }^{1}$ Northern Regional Genetics Service, Auckland City Hospital, Auckland, New Zealand ${ }^{2}$ Clinical Genetics Group, Otago University, Dunedin, New Zealand

'Direct-to-consumer' genetic testing has been offered in recent times through a number of overseas laboratories. This has been controversial, and criticisms have included a risk of misinterpretation by lay people who have submitted their own samples; a very limited practical usefulness of the results received; and a concern that basing an interpretation upon gradually accumulating GWAS data could be premature. But the genie is out of the bottle (or the DNA out of the spit tube), and genetic professionals cannot, in my view, decline to be involved, if people seek our advice (albeit that our advice may be rather cautious). I have tested the system by sending my own sample to San Francisco, and I will reveal some of my own findings. I will also comment upon a local (NZ) case of a woman who sent her sample for Alzheimer testing (I am supposing apoE), and who received her high-risk result while 60 Minutes' TV cameras were rolling. 


\section{USE OF THE NORFOLK ISLAND GENETIC ISOLATE TO IDENTIFY MIGRAINE SUSCEPTIBILITY GENES}

L. R. Griffiths', H. C. Cox', R.A. Lea', C. Bellis², M. Carless², T. D. Dyer², J. Charlesworth'², E. Matovinovic', J. Blangero²

${ }^{I}$ Genomics Research Centre, Griffith Health Institute, Griffith University Gold Coast, Australia

${ }^{2}$ Department of Genetics, Southwest Foundation for Biomedical Research, San Antonio, United States

Norfolk Island is a small volcanic land mass, situated in the South Pacific Ocean $\sim 1,500 \mathrm{~km}$ SE of Brisbane. The island was settled in 1856 by Pitcairn Islander's descended from a limited number of English Bounty mutineer and Tahitian founders, with $\sim 80 \%$ of the permanent adult residents able to trace ancestral lineage to the original founders. The aims of the present study were to characterise the demographics and molecular genetics of migraine in a large pedigree derived from the Norfolk Island genetic isolate and perform genomewide linkage and association screening to localise and identify migraine susceptibility gene. Migraine was diagnosed in accordance with International Headache Society guidelines and had an overall prevalence of $24 \%$, with approximately $33 \%$ of women and $12 \%$ of men affected. Linkage studies were undertaken using 377 founderrelated individuals and genotyping of 382 microsatellite markers at $\sim 10 \mathrm{cM}$ coverage across the genome. In addition, 285 core individuals from the pedigree were genotyped for a GWAS using the Illumina 610-Quad beadchip. Linkage results were analyzed using non-parametric variance component methods with analysis identifying a peak signal on chromosome $13 \mathrm{q} 33.1(P=.006)$ and trait component analysis also showing suggestive linkage to C13 for a combined photophobia and/or phonophobia phenotype $(P=.0009)$. Heritability and linkage-based association analysis was performed using the SOLAR program. GWAS results were adjusted for sex, age, admixture and inbreeding and SNPs prioritized based on both statistical and biological significance. Pedigree analysis indicated that the migraine phenotype had strong heritability $\left(\mathrm{h}^{2}=0.53, P=\right.$ .016). GWAS analysis and SNP prioritisation incorporating biological annotation implicated 13 SNPs in 9 genes as being associated with migraine risk at the gene-wide level. Interestingly, 4 of the SNPs tag haplotypes in a single block and one of the four SNPs confers a Thr-Ala amino acid change providing a plausible candidate variant for involvement in disease causation.

\section{WHOLE GENOME ARRAY RESULTS IN XLMR PATIENTS}

K. Fagan ${ }^{2,3}$, N. Bain ${ }^{3}$, M. Field', A. Hackett', , M. F. Hunter ${ }^{1,2}$

${ }^{I}$ Hunter Genetics, Hunter New England Health, Newcastle, Australia

${ }^{2}$ Department of Health, University of Newcastle, Australia

${ }^{3}$ Cytogenetics, John Hunter Campus, Pathology North, Newcastle, Australia

Introduction: The Genetics of Learning Disability service was established to investigate X-linked mental retardation (XLMR) families. To the authors knowledge there are no studies that have investigated XLMR using genome wide array. Whole genome array studies performed on 56 possible XLMR families detected 17 copy number changes of which 7 were clinically significant, 8 were considered non causative copy number changes and 2 are still incomplete. Methods: Probands with suspected XLMR were initially studied for mutations in 6 non-syndromic XLMR by high resolution melt and had arrayCGH performed on a microarray specific for the $\mathrm{X}$ chromosome. Subsequently, patients without a diagnosis were studied on the whole genome 60k ISCA design oligo array platform [BlueGnome]. Results: Whole genome microarray studies detected $12 \%$ clinically significant copy number changes. Only 1 family had an $\mathrm{X}$ chromosome abnormality and the remaining 6 families had an autosomal mutation. All copy number changes were maternally inherited. 2 families had identical abnormalities of the new $15 \mathrm{q} 13.3$ microdeletion syndrome and 2 families had the recurrent $16 \mathrm{p} 11.2$ microdeletion syndrome. Discussion: 1 of the 7 pedigrees was Xlinked with an X chromosome aetiology, the other 6 were compatible with X-linked inheritance but had an autosomal aetiology, although 4 of these could also have been interpreted as being compatible with
$\mathrm{X}$-linked, autosomal dominant or polygenic inheritance. We discovered 2 recurrent deletions, $15 \mathrm{q} 13$ and $16 \mathrm{p} 11$, which are well-recognized microdeletion syndromes and fit our patients phenotypes. Previous studies have shown no parent of origin bias for the $15 \mathrm{q} 13$ and 16p11 microdeletion syndromes. Conclusions: Physicians should consider whole genome array in XLMR families despite a convincing X-linked pedigree. Deletion 15q13 and 16p11, recurrent in our cohort, may mimic XLMR pedigrees.

\section{REIGER ANOMALY DUE TO A FOXC1 GENE DELETION: LOOK BEYOND THE EYES}

B. Hanna, S. Rajagopalan

SSWAHS, Liverpool Hospital, Sydney, Australia

Axenfeld-Rieger (AR) malformations are developmental defects of the anterior segment of the eye, associated with a high risk of glaucomatous blindness. A proportion of affected individuals have syndromic features (termed Axenfeld-Rieger syndrome or ARS), which include distinctive craniofacial dysmorphology, dental anomalies, umbilical herniae and redundant periumbilical skin. Heterozygous germline mutations of two genes, the forkhead transcription factor gene FOXC1 (6p25) and PITX2 (4q25) have been proven to cause ARS. Haploinsufficiency through deletion of FOXCl has also been reported in ARS, and this may be part of a contiguous $6 \mathrm{p} 25$ terminal or interstitial deletion. Contiguous deletions encompassing FOXC1 may also cause congenital heart disease, cerebellar and renal anomalies, hearing loss and developmental delay. This case report presents a 12-month old boy with bilateral Rieger anomaly. On initial assessment at age 7 months, he had striking dysmorphic features including microcephaly, plagiocephaly, telecanthus, hypertelorism, proptosis, malar hypoplasia, pre-auricular skin tags, widely spaced nipples and pectus excavatum. He had a normal male karyotype. Array CGH using 60k ISCA Oligoarray detected a de novo interstitial microdeletion spanning $1 \mathrm{Mb}$ in size in chromosome 6p25, which encompasses the FOXC1 gene and GMDS gene. The child is now receiving treatment for glaucoma and appears to be developing normally to date. We have recommended imaging of the central nervous system and audiology testing to clarify the presence of any extra-ocular manifestations as seen in $6 \mathrm{p} 25$ microdeletion syndromes. The presence of an Axenfeld-Reiger anomaly, in combination with a characteristic facial gestalt, should raise clinical suspicion of a chromosome $6 \mathrm{p} 25$ deletion encompassing the $\mathrm{FOXCl}$ gene. Investigation with a high-resolution array $\mathrm{CGH}$ is recommended to elucidate the genetic aetiology in these individuals, which in turn may help target specific surveillance for non-ocular manifestations.

\section{CLINICAL SUPERVISION: THE TYRANNY OF DISTANCE}

A. Hattam, J. McGaughran

Genetic Health Queensland, Royal Brisbane \& Women's Hospital, Brisbane, Australia

Genetic Health Queensland (GHQ) is a state-wide superspecialty service which offers genetic services throughout Queensland. The unit has a hub and spoke model with the majority of the clinical staff based in Brisbane and six genetic counsellors based in regional settings. Clinical supervision of genetic counsellors is part of the role of the Principal Genetic Counsellor at GHQ. This process is extremely important to ensure the maintenance of professional clinical standards within the service. Providing supervision to a number of counsellors in an outreach setting presents a challenge. In addition to facing barriers to professional development, regional counsellors face the risk of isolation, disempowerment and frustration due to a lack of local support. Effective supervision aims to minimise these risks. The traditional model of supervision to regional counsellors in Queensland has been a combination of regular telephone contact, face-to-face contact during annual GHQ Inservice and less frequent visits to the regional area. In an attempt to improve the process we have implemented the use of video-phones for clinical supervision of regional counsellors. Studies have suggested that a subjective richness of interaction via video compared with telephone interactions can reduce feelings of 
isolation. A qualitative analysis of the effectiveness of this system for regional counsellors in Queensland will be presented. The benefits and/or difficulties of using this approach will be highlighted and compared to the traditional model.

\section{USING GENOME SEQUENCE AND TRANSCRIPTION FACTOR DATA TO INTERPRET HUMAN DISEASE AND CLINICAL PHENOTYPE-ASSOCIATED GENETIC VARIATIONS}

sample was recruited from a broad range of sources. 87 participants completed a written questionnaire. Of these, 79 provided a saliva sample for genetic analysis and 77 agreed to take part in an interview. The mean age was 43 years (range 19 to 76 ). Compared to population normative data, there was strong evidence $(p<.001)$ of the KS cohort having poorer outcomes for: subjective wellbeing (PWI), body image (MBSRQ), self-esteem (RSE), and mental health (K-10). $72 \%$ of participants were diagnosed as adults, and $66 \%$ of these wished they had been diagnosed earlier. Two thirds of all participants supported population screening for KS. For men with KS, there is a measurably negative psychosocial impact of having this condition. The possible benefits of achieving earlier diagnosis through population screening should be considered in relation to possible negative impacts, using available frameworks ${ }^{1}$.

\section{UNDERSTANDING HIGH-GENETIC-RISK COUPLES DECISIONS REGARDING PREIMPLANTATION GENETIC DIAGNOSIS IN THE UNITED STATES: INSIGHT INTO FAMILY PLANNING STRATEGIES}

P. E. Hershberger', J.Wilbur' ${ }^{2}$ A. Schwartz', K. Kavanaugh'

${ }^{I}$ University of Illinois at Chicago, United States

${ }^{2}$ Rush University, United States

Background: Advances in genomic science have lead to an increasing ability to identify procreative couples who are at high-genetic-risk for transmitting an inherited disorder to their future offspring. There is little knowledge about how these couples decide to accept or decline Preimplantation Genetic Diagnosis (PGD), a contemporary reproductive option fraught with social and ethical controversy. Objective: To provide an in-depth description of the complexities and nuances involved with deciding to accept or decline PGD among a sub-group of high-genetic-risk couples in the United States. Methods: In this qualitative study, couples were recruited from a reproductive clinic and the Internet. Semi-structured interviews were conducted with each partner within the couple dyad. A sub-group of couples emerged that was ambivalent about undergoing PGD and was neither decisively for or opposed to using PGD. This group was identified for further descriptive analyses. Results: Of the 22 couples interviewed, $41 \%$ reported decision processes where they had or were planning to combine instances of accepting and declining PGD along with other family planning strategies. These couples described boundaries for PGD that included: (a) attempting natural or assisted conception first, followed by PGD; (b) intending to undergo PGD, but declining PGD and delaying conception in the immediate future for financial concerns; (c) opting for natural conception or adoption following an unsuccessful PGD cycle; and (d) forgoing PGD for procreation of their first offspring with future PGD use determined by the genotype of the resulting child(ren). Conclusions: For some couples, the dichotomous categories of accepting or declining PGD do not fully capture the complexity of their decision processes. Greater awareness and understanding of these underlying decision processes can inform clinicians, researchers, and policy makers of family planning strategies and salient concerns among high-genetic-risk couples.

\section{'A VERY SIMPLE THING TO DO': EXPERIENCES OF ADOLESCENTS AND THEIR PARENTS, OF PARTICIPATING IN GENETIC SCREENING FOR HEMOCHROMATOSIS IN VICTORIAN HIGH SCHOOLS}

C. Hickerton', E.Varley', M.Wolthuizen', K.Trembath', V. Collins', I. Macciocca ${ }^{3}$,

M. Aitken', L. Bond 1,2, K.Allen ',2,4, J. Craven', L. Gurrin',2, P. Lockhart', G.Wilson',

S. Stephenson', M. Delatycki ${ }^{1,2,5}$, S. Metcalfe ${ }^{1,2}$

${ }^{I}$ MCRI, Melbourne, Australia

${ }^{2}$ The University of Melbourne, Australia

${ }^{3}$ Genetic Health Services of Victoria, Melbourne, Australia

${ }^{4}$ Royal Children's Hospital, Melbourne, Australia

${ }^{5}$ Austin Health, Melbourne, Australia

Hereditary hemochromatosis $(\mathrm{HH})$ is a preventable iron overload condition. The ironXS study investigates acceptability and feasibility 
of genetic screening for $\mathrm{HH}$ in high schools, including exploring the experiences of teachers, adolescents and their parents. Year 10 and 11 Victorian students attended education sessions, completed baseline questionnaires and were offered screening for the HFE C282Y mutation. Students completed 1- and 12-month questionnaires and in-depth interviews were offered to all C282Y homozygotes, a sample of heterozygote and 'wild-type' students, and parents. Interview data were analyzed using content and thematic analysis. Briefly, 5758 students were tested (33\% uptake), identifying 28 C282Y homozygotes who all attended genetic counseling sessions with their parents, and $586 \mathrm{C} 282 \mathrm{Y}$ heterozygotes, who received their result by mail. 130 interviews were conducted: 64 students $(24$ homozygotes) and 66 parents. Participants' overall views of being offered a genetic test for $\mathrm{HH}$ at school were positive, regardless of their result. The most common reasons for having the test included: 'just to check'; to find out more about self; and to prevent HH. Most felt it was a simple decision and thought offering at school appropriate. Participants spoke of an increase in health awareness regarding: learning about $\mathrm{HH}$; awareness of health and preventing illnesses; learning about individual and family health. In many cases, heterozygous or homozygous results were unexpected; however these participants felt they had enough information and support to deal with this. Most participants indicated the result had minimal impact; a small, but important, group referred to positive experiences of cascade screening. Results suggest that adolescents are receptive to school-based genetic screening for $\mathrm{HH}$; this model of screening with school-based education plus clinical follow-up of homozygotes is acceptable to students and their parents. We are continuing to explore experiences of other stakeholders, such as teachers and those who chose not to participate.

\section{DISCUSSING FAMILIAL CANCER WITH THE CHINESE COMMUNITY: WHAT ARE THE BARRIERS IN ACCESSING FCC SERVICES?}

Y. Bylstra, L. Hodgkin

Genetics, Royal Melbourne Hospital, Australia

The Melbourne Health Familial Cancer Centre ('the FCC') provides a service to people in Victoria who have a personal and/or family history of cancer suggestive of an inherited cause. Heritable cancer syndromes affect all ethnicities, however, people from culturally and linguistically diverse (CALD) backgrounds do not attend the FCC in the numbers that reflect the diversity of the Victorian population. One group that is under-represented at the FCC is people of Chinese ethnicity. We aim to identify some of the barriers that may limit people from Chinese backgrounds accessing the FCC's services. The Chinese Cancer Society of Victoria (CCSV) supports people from a Chinese background 'whose lives are affected by cancer'. In October 2010, the CCSV is holding a 'Cancer Awareness Expo' to promote awareness about cancer prevention, and early detection in the Chinese community. Genetic counsellors at the FCC have been invited to participate in the Expo by staffing an information stall. To raise awareness of our service, we will provide the FCC information brochure in both traditional and simplified Chinese to all 250 Expo attendees. To facilitate communication exchange at the Expo, Mandarin and Cantonese interpreters will be available as needed. We plan to explore with Chinese attendees their possible unmet needs and any issues about cancer screening and/or genetic testing that may limit their access to the FCC. We will do this formally with questionnaires and informally by talking to individuals. Our findings will be discussed. We anticipate this will be one step towards providing a more culturally appropriate and equitable healthcare service to the Victorian Chinese community.
UNINTENDED DIAGNOSIS OF VON HIPPEL LINDAU SYNDROME USING ARRAY COMPARATIVE GENOMIC HYBRIDISATION (CGH)

J. Hogan', A. Turner ${ }^{1,2}$, K. Tucker ${ }^{1,3}$, L. Warwick'

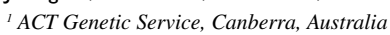

${ }^{2}$ Sydney Children's Hospital, Sydney, Australia

${ }^{3}$ Prince of Wales Hospital, Sydney, Australia

Array Comparative Genomic Hybridisation (CGH) is a powerful tool for identifying genomic imbalances and providing a diagnosis in individuals who have previously had a normal karyotype. It has been particularly useful in the investigation of individuals with developmental delay +/-, dysmorphic features and/or multiple congenital abnormalities. Array CGH has allowed the discovery of new genes and characterisation of new genetic syndromes and is becoming a routine test that is replacing the use of the karyotype. However, this non-targeted method of scanning the whole genome can produce results that are difficult to interpret and can also reveal unexpected information. We present a case in which the use of CGH array to identify the cause of a 17 year old boy's developmental delay found a de novo deletion of chromosome 3 p25.3. This deletion contains 25 genes and is likely to be the cause of the patient's phenotype. However also included in this deletion was the gene for Von Hippel Lindau (VHL) syndrome. Testing for VHL is usually only offered after extensive counseling about the implications of this condition. Scanning the genome by array CGH does not allow for this process and presents a number of challenges for the genetic counsellor. A search of the literature revealed no research has been done on the psychosocial effects of an unexpected genetic diagnosis on array CGH. We suggest that further understanding of the psychosocial effects of array CGH is needed to adequately provide pre and post test counseling.

\section{REFERRALS - THE VARIOUS PATHS FOR AN INDIVIDUALS} JOURNEY

B. Hopper', K. Frumar ${ }^{2}$, C. Sorensen ${ }^{2}$, J. Stofmeel ${ }^{3}$, G. Turner ${ }^{4}$

${ }^{1}$ Genetics, Hunter New England Area Health Service, Taree, Australia

${ }^{2}$ Genetics, 1 North Coast Area Health Service,, Port Macquarie, Australia

${ }^{3}$ Genetics, North Coast Area Health Service,, Coffs Harbour, Australia

${ }^{4}$ Genetics, Hunter New England Area Health Service, Newcastle, Australia

Genetic counsellors from three outreach genetic counseling services in the mid-north coast of NSW undertook to evaluate the referral source, clients only seen by Genetic Counsellors, those seen by Geneticists, as well as identifying time frames and reasons for referral from 2007-2009. This was for the 'general' client as opposed to 'cancer' clients; which is approximately half of the current client referrals. It was found that $50 \%$ of initial contacts to the genetic service are by an individual, and referral letters are organized later. $29 \%$ of referrals are seen by the Genetic Counsellor alone and illustrated in the flow chart. The three main reasons are pregnancy planning (23\%), support after a diagnosis (23\%), and cystic fbrosis $(17 \%)$. It is hypothesized that this could be increased. Clients who were seen by the genetic counsellor and by the geneticist were further evaluated to establish clinic outcome. $74 \%$ of clients under 18 years were referred for intellectual disability, and the use of microarray is expanded further by Prof Gillian Turner in her oral presentation at this conference. This flowchart maps the client's journey through the genetic clinic. This poster is part of larger project with 3 other oral presentations at this conference. 
HEALTH PROFESSIONALS' VIEWS ON PERINATAL HOSPICE CARE: IMPLICATIONS FOR GENETIC COUNSELING PRACTICE.

A. Horwood', S. Buscombe' ${ }^{2}$,. Hodgson ${ }^{1,3}$

${ }^{1}$ Department of Paediatrics, University of Melbourne, Australia

${ }^{2}$ Familial Cancer Centre, Peter MacCallum Cancer Centre, Melbourne, Australia

${ }^{3}$ Genetics Education and Health Research, Murdoch Children's Research Institute, Melbourne, Australia

Pregnant women receiving a diagnosis of a 'lethal fetal abnormality' may choose to either terminate their pregnancy, or continue the pregnancy in the knowledge that their baby is likely to die before or shortly after birth. In some countries, Perinatal Hospice Care is offered in these circumstances, comprising of medical care and psychosocial support provided by a multidisciplinary team. This caring environment allows families to engage with the ongoing pregnancy and value whatever time they have with their baby. This research explored the views and experiences of key informant health professionals in Victoria. The health professionals were involved in caring for these women about (a) the current practice of care for women who choose to continue a pregnancy diagnosed with a 'lethal fetal abnormality', and (b) the acceptability of Perinatal Hospice Care. Eight semi-structured interviews were analyzed using a qualitative approach allowing a number of themes to emerge. Focussing on the implications for the genetic counseling profession, this presentation will discuss the findings of this research, which included the view that current practice in Victoria is variable, commonly referred to as 'ad hoc', with some components needing improvement. The theme of a perceived 'turf war' between health professionals caring for these women arose from the research, along with the need to maintain hope for health professionals working in this area. A number of professional issues emerged including the need for education and support for professionals working in this area of practice. These research findings have practice implications for genetic counsellors and other health professionals involved in the care of women who choose to continue a pregnancy diagnosed with a 'lethal fetal abnormality'.

FIRST-TRIMESTER SCREEN BIOCHEMISTRY: ASSISTED REPRODUCTION VS. SPONTANEOUS REPRODUCTION

L. P. Hunt', A. Mclnerney-Leo' ${ }^{2}$ M. Peterson'

${ }^{\prime}$ Biomolecular and Physical Sciences, Griffith University, Australia ${ }^{2}$ so+gi, Brisbane, Australia

The combined first-trimester screen (cFTS) is a screening tool that primarily estimates the likelihood that the fetus is affected by trisomy 21 . The cFTS alters the maternal age-related risk by incorporating maternal serum blood biochemistry and ultrasound measurements. Smoking, maternal weight and ethnicity are known to affect the cFTS marker ranges, hence are incorporated into the algorithm that calculates the adjusted risk. The impact that assisted reproductive technologies (ART) and fetal sex have on the cFTS markers are not well understood, and are not currently taken into account. Objective: To identify if:

- biochemical markers, pregnancy-associated plasma protein-A (PAPP-A) and free beta-human Chorionic Gonadotropin (f $\beta-h C G)$ are influenced by ART

- ART are associated with an increased false-positive rate

- fetal sex impacts on the cFTS biochemical markers or falsepositive rate

- uptake of invasive testing differs between the natural and ART conceived population.

Method: Three ultrasound clinics from across south-east Queensland collaborated to provide 15716 de-identified cFTS cases, 2173 conceived through ART. All non-singleton pregnancies and those with diagnosed chromosomal or fetal abnormalities were excluded. Results: ART population have a statistically significant decrease in PAPP-A levels at the cFTS $(p<.001)$. False-positive rates were increased in the ART population (9.5\%) compared to the spontaneous population $(5.5 \%)(p=.057)$. PAPP-A and $\mathrm{f} \beta-\mathrm{hCG}$ were significantly higher in pregnancies with a female fetus compared to those carrying a male fetus $(p<.001)$, but had no significant impact on the false-positive rate. Women whose pregnancies were conceived through ART were less likely to have invasive testing ( $p=$ .001). Conclusion: Pregnancies conceived through ART in this research cohort have a higher false-positive rate for the cFTS compared to those conceived naturally. A meta-analysis confirming these findings would allow adjustments to the software algorithm. This could reduce unnecessary stress for people conceiving through ART.

\section{'NO THANKS' - WHY PREGNANT WOMEN CHOOSE NOT TO HAVE CYSTIC FIBROSIS CARRIER SCREENING}

L. Ioannou ${ }^{1,2}$, J. Massie ${ }^{4,5}$, S. Lewis ${ }^{3}$, M. Delatycki ${ }^{1,25,6,7}$

${ }^{\prime}$ Bruce Lefroy Centre, Murdoch Childrens Research Institute, Melbourne, Australia ${ }^{2}$ Department of Medicine, Monash University, Australia

${ }^{3}$ Public Health Genetics, Murdoch Childrens Research Institute, Melbourne, Australia ${ }^{4}$ Department of Respiratory Medicine, Royal Children's Hospital, Melbourne, Australia ${ }^{5}$ Department of Paediatrics, University of Melbourne, Australia

${ }^{6}$ Genetic Health Services Victoria, Melbourne, Australia

${ }^{7}$ Clinical Genetics, Austin Health, Melbourne, Australia

A population-based cystic fibrosis (CF) carrier-screening program was introduced in Victoria, Australia in 2006, and offered to couples planning a pregnancy or in early pregnancy from their doctor for a fee. The aim of this study was to assess the attitudes of women declining screening. Between December 2009 and August 2010, 256 women were approached in the waiting rooms of two private ultrasound clinics in Melbourne. Fifty-nine women $(23 \%)$ had received an offer of screening and $20(7.8 \%)$ choose to be screened. All women who declined screening $(n=39)$ were invited to participate. The questionnaire developed explored knowledge, reasons for declining screening, decision-making, attitude towards screening and satisfaction with information provided. Twenty-seven women (71\%) returned the questionnaire with the majority aged 30-34 (65\%) and having screening for Down syndrome (92\%). Eleven (44\%) answered 10 to 13 of the 15 knowledge questions correctly; however $65 \%$ incorrectly believed that couples who have a child with $\mathrm{CF}$ usually have a family history. The main reasons for declining screening were having no family history and having previously had healthy child/children. Cost was not an influential factor when deciding to have screening for most women $(58 \%)$. While the majority of women were satisfied with their decision not to have screening, $16 \%$ regretted their decision and $20 \%$ would have changed their decision. Twenty women (78\%) felt they had enough information to make a decision in regards to having screening, with only eight women (31\%) seeking further information. All women believed that $\mathrm{CF}$ carrier screening should be available to those who wish to have it. In conclusion, our study demonstrates that most women in the private sector are not being offered CF screening. The main reason women choose not to have screening is the absence of a family history of $\mathrm{CF}$ and previous healthy child/children.

EVALUATION OF A MULTI-DISEASE CARRIER SCREENING PROGRAM IN ASHKENAZI JEWISH HIGH SCHOOLS

L. Ioannou ${ }^{1,2}$, J. Massie ${ }^{4,5}$, S. Lewis ${ }^{3}$,V. Petrou ${ }^{6}$, A. Gason ${ }^{1,7}$, S. Metcalfe ${ }^{5,7}$,

M.Aitken ${ }^{5,8}$, A. Bankier ${ }^{6}$, M. Delatycki ${ }^{1,25,5,9}$

${ }^{I}$ Bruce Lefroy Centre, Murdoch Childrens Research Institute, Melbourne, Australia

${ }^{2}$ Department of Medicine, Monash University, Australia

${ }^{3}$ Public Health Genetics, Murdoch Childrens Research Institute, Melbourne, Australia

${ }^{4}$ Department of Respiratory Medicine, Royal Children's Hospital, Melbourne, Australia

${ }^{5}$ Department of Paediatrics, University of Melbourne, Australia

${ }^{6}$ Genetic Health Services Victoria, Melbourne, Australia

${ }^{7}$ Genetics Education and Health Research, Murdoch Childrens Research Institute, Melbourne, Australia

${ }^{8}$ Research Strategy, Murdoch Childrens Research Institute, Melbourne, Australia ${ }^{9}$ Clinical Genetics, Austin Health, Melbourn, Australia

A high school Tay Sachs disease carrier screening program was introduced in Victoria, Australian in 1997, and was expanded to screen for six other genetic conditions that are common in the Ashkenazi Jewish population in 2008. The aim of this study was to 
evaluate the current program and compare it to an evaluation of the program when screening was offered for Tay Sachs disease alone. All students from Jewish high schools in Melbourne who were part of the program in 2009 were invited to participate in the study. A purpose-designed questionnaire explored the following domains: knowledge (disease and genetics), reasons for having screening, anxiety and predictive negative feeling if found to be a carrier. Two hundred and seventy-three students were offered screening and 272 $(99.6 \%)$ completed the questionnaire. Only two students choose not to have screening. Two hundred and seventy one students were in the second last year of high school (99.6\%) and 222 were of Ashkenazi Jewish descent $(82.5 \%)$. The main reasons for choosing screening were; wanting to know carrier status and convenience. Knowledge level was decreased and negative feelings were increased in the current cohort compared to when screening was offered for Tay Sachs disease alone. We conclude that the current program is efficient, although increasing the number of conditions resulted in a decrease in knowledge and increase in predicted negative feelings if found to be a carrier of one of the conditions. This has implications for all multi-disease screening programs that will increase in frequency as more conditions can be screened for and costs diminish.

\section{CLINICAL APPLICATIONS OF THE GENOMIC VARIANTS ASSOCIATED WITH BREAST CANCER IN HIGH RISK HEREDITARY BREAST CANCER FAMILIES}

\author{
P.A. James', S. Sawyer', J. McKinley', G. Chenevix-Trench', M. Harris ${ }^{3}$, \\ G. Lindeman', G. Mitchell' \\ ${ }^{\prime}$ Familial Cancer Centre, Peter MacCallum Cancer Centre, Melbourne, Australia \\ ${ }^{2}$ Familial Cancer Centre, Royal Melbourne Hospital, Melbourne, Australia \\ ${ }^{3}$ Familial Cancer Centre, Southern Health, Melbourne, Australia \\ ${ }^{4}$ Queensland Institute of Medical Research, Brisbane, Australia
}

Background: Genome-wide association studies (GWAS) in the general population have identified common susceptibility alleles that are associated with breast cancer risk. A direct clinical application for this SNP data in the general population is controversial but greater risk effects for the same SNPs in the context of a strong family history suggests that analysis of common variants may have a clinically important role in the Familial Cancer setting. Aim: To study the distribution, predictive qualities and clinical associations of an extended panel of validated breast cancer susceptibility variants in probands from high-risk Victorian breast cancer families and assess the clinical utility of this information. Methods: Probands were identified from 1450 families who were assessed by a Familal Cancer Centre (FCC) as high risk for hereditary breast cancer and had completed BRCA1 and BRCA2 mutation screening (136 individuals with BRCA1 and 130 with BRCA2 mutations). Genotyping was performed for 25 predisposition SNPs. Data was compared to 1068 controls and examined for correlations with personal and family data. Results: The familial breast cancer cohort showed a highly significant increase in breast cancer risk alleles $\left(p=3.1 \times 10^{-13}\right)$. Higher ORs for most SNPs compared to population based data resulted in increased accuracy of predicted breast cancer risk compared to previous reports; RR 1.4$2.5 \times$ population incidence rates in the highest risk quintile, with a significant increase in bilateral and very early onset cases. The distribution of risk alleles in BRCA1 and 2 patients was not different to the control population, so that SNP data strongly improved the prediction of the presence of high penetrance gene mutations compared to family history alone (AUC ROC 0.87 vs 0.77 ). Conclusions: Data on common variants in an FCC based cohort defines a clinically important group of 'common variant risk' patients and provides and improved assessment of breast cancer aetiology.

\section{AN UNUSUAL QF-PCR RESULT — XQ28 DUPLICATION} OR A RARE POLYMORPHISM?

\author{
A. M. Jay', A. E. Brown',A. N. Beddow', K. J. Flintoff', C.A. Felix' \\ Cytogenetics, Central and Southern Regional Genetic Service, Wellington, New Zealand \\ ${ }^{2}$ Molecular Genetics, Central and Southern Regional Genetic Service, Wellington, \\ New Zealand
}

An amniocentesis sample was received from patient KM due to detection of a two-vessel cord and cysterna magna on ultrasound scan. A small aliquot of this sample was processed for Rapid Aneuploidy Detection ( RAD ) by QF- PCR. 23 of the 24 markers gave a female result with no aneuploidy detected. However an unusual result was seen for the marker X22, which localises to Xq28 and Yq11.2. This marker showed 3 peaks indicating the presence of 3 different alleles in this region. The 5 other $\mathrm{X}$ chromosome markers showed 2 peaks, and there was no evidence of a Y chromosome with either SRY, AMEL or the DYS448 markers. FISH studies were performed on fixed metaphase and interphase cells to exclude the possibility of either a duplication in the X22 region at Xq28 or of an unbalanced segregant from a $\mathrm{t}(\mathrm{Xq} ; ?)$ or $\mathrm{t}(\mathrm{Yq} ; ?)$ translocation, resulting in 3 copies of the $\mathrm{X} 22$ locus. These studies gave a normal result. In view of these findings, parental bloods were requested, which showed that the father of the pregnancy carried the same X22 polymorphism. This polymorphism is a rare finding but has been reported in the literature by Cirigliano et al., where it was observed $6 / 18000$ patients. This case highlights the fact that any molecular assay can unmask rare variants that require parental follow-ups to confirm that they are familial polymorphisms.

\section{EVALUATION OF EPIGENETIC DISCORDANCE IN HUMAN UMBILICAL VEIN ENDOTHELIAL CELLS (HUVECS) FROM NEWBORN MZ TWINS}

E.J.Joo', L. Gordon', M. Ollikainen', R.Andronikos', G. Smyth ${ }^{3}$, A. Oshlak ${ }^{3}$, R. Saffery', J. Craig'

Developmental Epigenetics, Murdoch Childrens Research Institute, Melbourne, Australia

${ }^{2}$ Bioinformatics, Murdoch Childrens Research Institute, Melbourne, Australia

${ }^{3}$ Bioinformatics, Walter and Eliza Hall Institute, Melbourne, Australia

Monozygotic (MZ) twins are widely considered to share an identical genetic profile as they are derived from a single fertilized zygote. In light of this, it has been assumed that environmental factors are responsible for the phenotypic differences that occur within most MZ pairs. Recently, evidence has emerged that epigenetics may play a role in the mechanism underlying environmental influence on the underlying genome, potentially explaining the phenomenon of 'fetal programming', linked to increased disease risk later in life. DNA methylation is the most widely studied epigenetic mechanism and also known to be sensitive to environmental influences such as diet and lifestyle. A study by Fraga et al. showed that MZ twins show increasing levels of DNA methylation discordance with age, with greater differences seen in pairs who have shared less of their environment over their lifetimes. However, little data are available on the level of epigenetic change accumulated in utero. We hypothesise that fetal development represents a particularly sensitive time for environmental influence on epigenetic marks, and that differences in epigenetics and gene expression at birth will reflect differences in intrauterine microenvironment. To test this hypothesis, we examined genome-wide methylation and expression profiles, using Illumina Infinium methylation arrays and expression arrays, in purified populations of HUVECs collected at birth from $10 \mathrm{MZ}$ pairs. These cells represent an excellent model for studying factors that may program later disease risk associated with suboptimal endothelial function in humans. We observed a range of within-pair expression and methylation discordances and have characterized a set of genes that differ most widely in expression and methylation within pairs that are known to play a role in response to environment. We have also characterized a set of genes associated with birth weight, many of which have previously been linked to cardiovascular disease. 
EXPRESSION PROFILING IDENTIFIES BIOMARKERS FOR PROGRESSION TO TYPE 2 DIABETES

C.Attard', N. Cummings', J. Curran', K. Shields', E. Aplin', O. Ramaraj', M. Johnson', H. Goring', T. Dyer', P. Zimmet', E. Moses², J. Blangero', J. Jowett'

${ }^{\prime}$ Genomics \& Systems Biology, Baker IDI Heart and Diabetes Institute, Melbourne, Australia

Genetics, Southwest Foundation for Biomedical Research, San Antonio, United States

The development of most common chronic diseases is influenced by heritable genetic factors that increase or decrease an individual's propensity to develop the disease. The identification of biomarkers that are influenced by these factors could form the basis upon which to quantify an individual's risk of disease and allow preventive action to be prescribed as appropriate. Additionally, knowledge of the nature of these physiological perturbations may reveal novel modalities for therapeutic intervention. To identify biomarkers related to progression to type 2 diabetes we undertook a large-scale gene expression profiling study within a family cohort structure that allows discrimination between variations that are influenced by heritable factors from those that are sporadic. We quantitated 47,289 transcripts in 1,240 individuals from 42 extended family pedigrees in lymphocyte samples as surrogate measures of gene expression in disease relevant tissues. This approach has been receiving increased attention in cardiac and renal allograft rejection, onset of type 1 diabetes and CNS disorders that point to its potential utility and success. Using quantitative correlates of diabetes risk, we identified a set of secreted proteins and demonstrate in an independent longitudinal cohort that these proteins differ in individuals who progress to type 2 diabetes as compared to those that remain normal glucose tolerant. The best predictors are involved in modulation of immune function and chronic inflammation.

\section{IMPROVEMENT OF CENTRAL NERVOUS SYSTEM PATHOLOGY IN MURINE MUCOPOLYSACCHARIDOSIS IIIA WITH GANGLIOSIDE TARGETED SUBSTRATE REDUCTION THERAPY}

X. Kaidonis ${ }^{1,2}$,A. L.K. Derrick Roberts ${ }^{1,3}$, J. M. Fletcher ${ }^{1,3}$, S. Byers ${ }^{1,2,3}$

${ }^{1}$ Genetics and Molecular Pathology, SA Pathology, Adelaide, Australia

${ }^{2}$ Genetics, The University of Adelaide, Australia

${ }^{3}$ Paediatrics, The University of Adelaide, Australia

Introduction: Mucopolysaccharidosis IIIA (MPS IIIA) is an inherited disorder, in which degradation of a complex carbohydrate extracellular matrix component, heparan sulphate, is impaired. This results in progressive central nervous system (CNS) degeneration for which there is no currently available effective therapy. An additional secondary storage of gangliosides $\mathrm{G}_{\mathrm{M} 2}$ and $\mathrm{G}_{\mathrm{M} 3}$ occurs in the MPS IIIA brain, and is believed responsible for the CNS pathology characteristic of the disease. This study tests the iminosugar $\mathrm{N}$-butyldeoxynojirimycin (NB-DNJ) to specifically inhibit storage of $\mathrm{G}_{\mathrm{M} 2}$ and $\mathrm{G}_{\mathrm{M} 3}$ in the brain of the MPS IIIA mouse. Aim: To investigate whether targeting the secondary storage of ganglioside in a murine model of MPS IIIA using NB-DNJ will improve CNS pathology. Methods: Mice were treated with intravenous NB-DNJ 3 times per week from 4 weeks of age and their CNS function tested. Anxiety and exploratory behavior was measured at 2, 4 and 6 months using the open field test. Spatial learning at 6 months was tested in the water cross-maze. Results: MPS IIIA mice displayed a $6 \%$ and $10 \%$ reduction in anxiety at 4 and 6 months respectively. This deficit was corrected to normal levels in $N B-D N J$ treated mice. Spatial learning of MPS IIIA mice was reduced by $17 \%$, while learning in treated mice followed normal trends. Conclusion: These results support the theory that ganglioside accumulation contributes to CNS pathology in MPS IIIA, and demonstrate the potential for SRT by NB-DNJ to improve quality of life of affected patients.
GJB2 MUTATION SPECTRUM AMONG AUSTRALIAN PATIENTS WITH HEARING IMPAIRMENT

M. Kaps, D. Phelan, V. Calabro, S. Pantaleo, D. Du Sart

Molecular Genetics, VCGSPathology, Murdoch Childrens Research Institute, Melbourne, Australia

Hearing impairment is the most common sensory defect occurring in approximately 1 in 1000 newborns. Genetic causes of hearing loss are quite heterogeneous and to date 42 genes associated with nonsyndromic deafness have been identified. Among them, gap junction beta 2 (GJB2), is the major cause of autosomal recessive non-syndromic hearing loss in many populations. The GJB2 gene, encoding connexion 26 (Cx26), is located on chromosome 13 and contains a 681 bp coding domain in exon 2 . Mutations in this gene are associated with both recessive (DFNB1) and dominant (DFNA3) forms of non-syndromic deafness. Although GJB2 is a small gene with only a single coding exon, more than 150 different pathogenic mutations, polymorphisms and unclassified variants have been described to date. The mutation spectrum and prevalence of mutations also vary significantly among different ethnic groups. Three mutations, c. $35 \mathrm{delG}$, c. $167 \mathrm{delT}$ and c. $235 \mathrm{delC}$ are the most frequent mutations in Caucasian, Ashkenazi Jewish and Asian populations, respectively. As a consequence, there are patients in which there is uncertainty about the clinical significance of identified mutations. These cases emphasize the need to test other family members and assess the presence of any clinical phenotype in those individuals. Our current method for diagnostic testing is to sequence this entire coding region of the GJB2 gene. Although c.35delG is known to account for up to $55 \%$ of mutant alleles seen in Australian patients (Dahl et al, 2001), the full spectrum of mutations occurring in the GJB2 gene is not well known. The aim of this current study is to investigate the spectrum of GJB2 mutations among hearing impaired Australians tested over the past 8 years (2003-2010). We will present the mutation spectrum, estimate the prevalence of these mutations within the Australian population and compare these findings with published data from other countries.

\section{A PROSPECTIVE STUDY ASSESSING ANXIETY, DEPRESSION AND MATERNAL-FETAL ATTACHMENT IN WOMEN USING PGD}

J. C. Karatas', K. Barlow-Stewart ${ }^{2}$, B. Meiser ${ }^{3}$, C. McMahon ${ }^{4}$, K. A. Strong ${ }^{2}$,

W. Hill' ${ }^{5}$, C. Roberts ${ }^{5}$, P. Kelly ${ }^{7}$

${ }^{I}$ Northern Clinical School, University of Sydney, Australia

${ }^{2}$ Centre for Genetics Education, NSW Health, Sydney, Australia

${ }^{3}$ Prince of Wales Clinical School, University of New South Wales, Australia

${ }^{4}$ Macquarie University, Department of Psychology, Australia

${ }^{5}$ Sydney IVF, Sydney, Australia

${ }^{6}$ The Centre for Values, Ethics and Law in Medicine, University of Sydney, NSW, Australia

${ }^{7}$ Sydney School of Public Health, University of Sydney, Australia

Abstract: Participants were enrolled in a prospective study exploring the psychological impact of preimplantation genetic diagnosis (PGD). Women seeking PGD treatment were asked to complete questionnaires prior to treatment; after embryo transfer; after pregnancy test result; and, for those who achieved pregnancy, at 24 weeks of pregnancy. A range of psychometric scales were included assessing anxiety, depression and, where appropriate, maternal-fetal attachment. Results: All women were married and on average had high yearly income as well as high education levels, with the majority of the women having university education. Overall, women's anxiety and depression scores were not significantly different to normative data. Nevertheless, $18 \%$ and $6 \%$ of women had symptoms of anxiety and depression, respectively, at levels of clinical significance. Using multivariate analysis, the degree of worry about the financial aspects of PGD, as well as living in an inner metropolitan area, were significantly associated with anxiety. Compared to prior to treatment, anxiety significantly increased following embryo transfer and following pregnancy test notification, regardless of whether the result was positive or negative. Women who received a negative pregnancy result were not significantly more anxious than women 
who did achieve a pregnancy. At 24 weeks of pregnancy, anxiety levels did not differ from baseline levels of anxiety. Levels of depression did not fluctuate significantly over the course of PGD. The cyclical nature of women's PGD pathways was an unexpected finding in this study. However, despite the often-difficult reproductive histories and the complex process of establishing a pregnancy with PGD, at 24 weeks of pregnancy maternal-fetal attachment was sound, compared to available normative data.

\section{HEREDITARY NON-POLYPOSIS COLORECTAL CANCER AND FAMILIAL ADENOMATOUS POLYPOSIS: INFORMATION DAYS FOR FAMILIES}

M. Kentwell'*, E. Lynch ${ }^{2,3 *}$, F. Macrae ${ }^{1,4}$, A. Boussioutas ${ }^{5}$, L. Lipton', E. Sadek ${ }^{6}$

${ }^{\prime}$ Familial Cancer Centre, Royal Melbourne Hospital, Melbourne, Australia

${ }^{2}$ Austin Health Genetics Service, Melbourne, Australia

${ }^{3}$ Genetic Health Services of Victoria, Royal Children's Hospital, Melbourne, Australia

${ }^{4}$ Department of Colorectal Medicine \& Genetics, Royal Melbourne Hospital, Melbourne, Australia

${ }^{5}$ Familial Cancer Centre, Peter MacCallum Cancer Centre, Melbourne, Australia ${ }^{6}$ Victorian Cooperative Oncology Group, The Cancer Council of Victoria, Melbourne, Australia

* Joint First Authors

Individuals with Hereditary Non Polyposis Colorectal Cancer (HNPCC) and Familial Adenomatous Polyposis (FAP) require ongoing medical screening to reduce the high risk of developing cancer. It has been suggested longer-term psychosocial support may also be necessary to address the ongoing challenges faced by this group. Inspired by a presentation at the 2005 InSIGHT meeting by St Mark's Hospital in the U.K, FCCs across Victoria and the Cancer Council of Victoria (CCV) jointly hosted information days for patients and their families with HNPCC and FAP in 2007 and 2009 respectively. A planning committee consisting of genetic counsellors, a geneticist, an oncologist, a consumer and a gastroenterologist developed programs for the information days. The programs included patient stories, information on genetics and medical management, current and future research information, psychosocial presentations, and discussion groups amongst the attendees facilitated by genetic counsellors. The four Victorian FCCs mailed invitations provided by the CCV to all of their patients recorded as being part of HNPCC or FAP families with known mutations. 32 people attended the FAP information day and 19 attended the HNPCC information day. An evaluation questionnaire by the CCV showed both days were well received by those who attended. In the FAP group, the attendees indicated the most valuable aspects of the day were meeting others with FAP, and information on diet and lifestyle following colectomy. In the HNPCC group, the attendees indicated the most valuable aspects of the day were meeting others with HNPCC and update on new information. The attendees also identified additional issues to discuss at future information sessions.

\section{SCREENING FOR PRIMARY CONGENITAL HYPOTHYROIDISM: COMPARISON OF FLUOROIMMUNOASSAY WORK PLATFORMS}

W. Kim, V.Wiley, B.Wilcken

NSW Newborn Screening Programme, Children's Hospital at Westmead, NSW, Australia

Background: Primary congenital hypothyroidism $(\mathrm{CH})$ leads to growth and mental retardation and affects at least 1 in 3000-5000 newborn babies. Early diagnosis and treatment provides normal development. $\mathrm{CH}$ can be detected by measurement of thyroid stimulating hormone ( TSH ) and has been included in newborn screening programmes since the 1970 s. In NSW, using a batch mode AutoDELFIA fluoroimmunoassay, the processing time for an average 400 samples per day is 10 hours. The number of samples received per day varies from 250 to 1200 ; therefore modifications to the method and instrumentation were required to ensure completion within a routine working day. The performance of two automated instrument platforms was compared. Method: As part of routine newborn screening, dried blood spot samples were collected at 48-72 hours of age from 20,000 newborns. TSH was estimated by solid phase, two site fluoroimmunoassay using an AutoDELFIA and in parallel on a continuous load Genetic Screening Platform (GSP) instrument. Samples from 160 babies with TSH >10mIU/LWB from archival storage were also assayed on both instruments. Result: Using the AutoDELFIA results were not available for release until the next working day, while GSP provided results the same working day as sample receipt. Values obtained using the GSP were lower than those using the AutoDELFIA more noticeably at higher concentrations ( $\mathrm{TSH} 0.1$ to 10 ratio 0.94 ; $\mathrm{TSH}>10=0.71$ ). Twenty babies with proven $\mathrm{CH}$ were detected by both instruments. Conclusion: The GSP provides higher sample capacity, has less turnaround time allowing faster time to treatment for babies with $\mathrm{CH}$ without loss of population sensitivity and specificity.

\section{CONFIRMATION AND NARROWING OF MULTIPLE QUANTITATIVE TRAIT LOCI MODIFYING ATRIAL SEPTAL MORPHOLOGY}

E. P. Kirk ${ }^{1,2,3}$, M. Moradi Marjaneh ${ }^{3}$, P. C. Thomson ${ }^{4}$, M. F. Buckley ${ }^{5}$, I. C.A. Martin',

C. Moran ${ }^{4}$, R. P. Harvey ${ }^{3}$

${ }^{\prime}$ Dept of Medical Genetics, Sydney Children's Hospital, Sydney, Australia

'School of Women's and Children's Health, University of New South Wales, NSW,

Australia

${ }^{3}$ Victor Chang Cardiac Research Institute, Sydney, Australia

${ }^{4}$ Centre for Advanced Technologies in Animal Genetics and Reproduction,

University of Sydney, Australia

${ }_{5}$ Molecular and Cytogenetics Unit, South Eastern Area Laboratory Services, Sydney, Australia

Atrial septal defect (ASD) is a common form of congenital heart disease (CHD), occurring in 5-7/10,000 live births. Like other forms of CHD, ASD has a strong genetic basis. However, Mendelian forms account for only a small proportion of cases, with the remainder having multifactorial causation. Human and mouse data suggest that ASD exists in an anatomical continuum with patent foramen ovale (PFO). We have previously shown in inbred mice that incidence of PFO strongly correlates with atrial septal anatomical features including the length of the interatrial septum primum. In earlier work, we used an F2 intercross approach to map 7 significant and 6 suggestive QTL influencing three quantitative septal traits. Here, we report fine mapping of seven of these QTL using an advanced intercross line (AIL). Starting with the parental strains from the F2 study, QSi5 and 129T2/SvEms, we bred 14 generations of mice. The goal of breeding in the AIL is to maximise recombinations while avoiding loss of genetic information through inbreeding and genetic drift. 1,003 F14 mice were dissected. Of these, 400 were selected for genotyping, using a combination of selection for the extremes of the three traits in question (180 mice) and random selection. 167 single nucleotide polymorphisms (SNPs) were chosen based on Mouse Hapmap data, with marker selection aiming to span the selected QTL (and an additional heart weight QTL). Six of the septal morphology QTL found in the F2 study were confirmed, with the interval covered by the QTL being considerably narrowed in each instance. One new significant QTL, on MMU2, was identified, and the QTL on MMU9 resolved into at least two, and possibly three, separate peaks. These findings will facilitate identification of the genetic basis of PFO in mice, and by extension should shed light on the causation of ASD in humans.

\section{MODELING OXPHOS DISORDERS USING NDUFS4 $4^{-/}$MICE \\ J. C. Komen', M.Yap', K.A. Miller², A. Laskowski', J. J. Pitt', M. Mckenzie, \\ M.T. Ryan ${ }^{3}$, H. M. Dahl' ${ }^{2}$, D. R.Thorburn ${ }^{1,4,5}$ \\ Mitochondrial Research, Melbourne, Australia \\ ${ }^{2}$ Genetic Hearing Research, MCRI, Melbourne, Australia \\ ${ }^{3}$ Biochemistry, La Trobe University, Australia \\ ${ }^{4}$ VCGS Pathology, MCRI, Melbourne, Australia \\ ${ }^{5}$ Pediatrics, University of Melbourne, Australia}

Mitochondrial oxidative phosphorylation (OXPHOS) diseases including Complex I deficiency are the largest group of inborn errors of metabolism. One of the main reasons for the paucity of effective therapies for patients with OXPHOS diseases is the lack of relevant experimental animal models for studying pathogenicity and trialing 
treatments. Ndufs $4^{-1}$ mice lack the Ndufs 4 subunit of OXPHOS Complex I resulting in a severe Complex I defect. On a C57BL/6 background the mice develop temporary fur loss, impaired growth, visual/hearing defects, unsteady gait, limb grasping when suspended by the tail, and ultimately die around P40. The phenotype of these mice resembles the disease symptoms observed in children with Complex I deficiency but the severity of the phenotype limits the time for a potential treatment study. We have backcrossed the $N d u f s^{-1}$ allele to mice with the BALB/c background and found that this mildly ameliorated the severity of the phenotype. Preliminary data have shown that $N d u f s 4^{-/}$BALB/c mice have less to no fur defects and develop severe symptoms at least one week later than their C57BL/6 counterparts. Auditory Brainstem Response measurements at different timepoints showed that mice gradually lost their hearing with hearing at week 4 still near wildtype levels. Metabolite analysis had previously shown elevated levels of hydroxyacylcarnitines suggesting a decrease in mitochondrial $\mathrm{NAD}^{+}$levels. We now have preliminary data showing that $\mathrm{NAD}^{+} / \mathrm{NADH}$ levels in multiple tissues are affected by the Complex I defect. In conclusion, we believe that $N d u f s 4^{-1}$ mice are a good model for Complex I deficiency in humans, especially on a BALB/c background. Data generated with the Ndufs4 ${ }^{-}$mice so far provide an excellent starting point for future development and testing of new therapies for OXPHOS disease for which currently none exist.

\section{THREE-DIMENSIONAL QUANTIFICATION OF FACIAL PROFILES OF INDIVIDUALS WITH SYNDROMES}

\footnotetext{
S. Kung', M.Walters' ${ }^{2}$, P. Claes ${ }^{3}$, G. Baynam ',4, P. Le Souef'

${ }^{1}$ School of Paediatrics and Child Health, The University of Western Australia, Perth, Australia

${ }^{2}$ Cranio-Maxillo-Facial Unit, Princess Margaret Hospital for Children, Perth, Australia ${ }^{3}$ School of Dental Science, The University of Melbourne, Australia

${ }^{4}$ Genetic Services of Western Australia, Princess Margaret Hospital for Children, Perth, Australia
}

Objectives: Recognition of atypical facial features in individuals with syndromes is challenging. We propose a novel multidimensional facial anthropometric tool to discriminate facial phenotypes. We are developing a facial registry of individuals with diagnosed syndromes that will be compared to our extant and expanding control population based reference datasets. Methods: A high dimensional statistical face space was generated from 3-dimensional (3D) facial profiles of individuals (880) aged 4-25 years, describing the limits of typical facial variation in our population. 'Normalized' synthetic faces are generated from patient data based on covariance of geometrical facial harmonies found in the normal range. This is robustly mapped to the diagnostic scan and dense quasi-landmarks (anthropometric mask) are applied to enable statistical comparisons. Facial differences are visualized in terms of statistical divergence (confidence limits), distance and vector maps between corresponding landmarks. This study undertook 'normal equivalent' comparisons of a Treacher-Collins and a Hunter syndrome patient. Results: Atypical facial variants consistent with previously described phenotypes were identified in both individuals, along with potential new discriminatory features. This technique requires further validation but promises to provide sensitivity and specificity in detecting phenotypic variants associated with craniofacial syndromes. Conclusion: This study supports the use of 3D quantification of facial profiles to discriminate facial variants in early syndrome diagnosis, particularly in individuals with milder facial forms, to allow timely therapeutic intervention. This may contribute in generating treatment monitoring and educational tools. Further studies are underway and nature of this work invites collaboration.

\section{MUTATION OF THE N-TERMINAL ACTIN-BINDING DOMAIN OF FILAMIN C (FLNC) CAUSE A DISTINCT DISTAL MYOPATHY}

R. M. Duff',2,V.Tay ${ }^{3}$, G. Ravenscroft', C. McLean ${ }^{4}$, P. Kennedy ${ }^{4}$, P.Van Der Ven ${ }^{5}$, D. Furst ${ }^{5}$, K. Reardon ${ }^{3}$, D. R. Williams ${ }^{6}$, J. Landers 7 , R. H. Brown', B. Udd ${ }^{8,9}$, K. J. Nowak', N. G. Laing'

${ }^{\prime}$ Centre for Medical Research, University of Western Australia, Australia

${ }^{2}$ Centre for Neuromuscular and Neurological Disorders, University of Western Australia, Australia

${ }^{3}$ St Vincent's Hospital, Melbourne, Australia

${ }^{4}$ State Neuropathology Service, The University of Melbourne, Australia

${ }^{5}$ Institute for Cell Biology, University of Bonn, Germany

${ }^{6}$ Van Cleef Roet Centre for Neurological Disease, Monash University, Australia

${ }^{7}$ Department of Neurology, Massachusetts General Hospital, Charlestown, United States

${ }^{8}$ Neuromuscular Research Centre, University of Tampere, Finland

${ }^{9}$ Folkhalsan Institute of Genetics, University of Helsinki, Finland

Linkage analysis of the distinct dominant distal myopathy we previously identified in a large Australian family, demonstrated one significant linkage region, encompassing $18.6 \mathrm{Mbp}$ and 151 genes, on chromosome 7 . The most likely candidate gene in the region was deemed filamin $\mathrm{C}$ (FLNC) because of its muscle-specific expression and previous association with myofibrillar myopathy. Sequencing the FLNC cDNA identified a novel p.Met251Thr mutation in the Nterminal actin-binding domain (ABD) which segregated with the disease $(\mathrm{LOD}=3.31)$ and was not seen in 200 controls. FLNC ABD mutations have not previously been described, though filamin A and B (FLNA and FLNB) ABD mutations have. The distal myopathy phenotype and muscle pathology in the family differs from the myofibrillar myopathies caused by $F L N C$ rod and dimerization domain mutations. Thus, like FLNA and $B$, the position of the $F L N C$ mutation determines the disease phenotype. We demonstrated that the FLNC ABD mutation increases actin-binding affinity, similar to FLNA and FLNB ABD mutations. Tissue culture expression of the mutant filamin $\mathrm{C}$, filamin A and filamin B actin binding domains demonstrated reduced nuclear localization of the mutant filamin $\mathrm{C}$ actin binding domain and all of the mutant filamin A and B actin binding domains tested. We conclude FLNC ABD mutations cause a distinct, recognizable distal myopathy most likely through increased actin affinity.

\section{A COMPLEX REARRANGEMENT RESULTING IN A 22 MB DELETION AT 11014. -11022 IN A 3-MONTH-OLD CHILD WITH SCLERODERMA DEVELOPMENTAL DELAY AND FAILURE TO THRIVE}

R. Lane', J. Peverall', D. Kennedy',V. Marchin',V. Britton', E. Harris², M. Ashleigh

${ }^{\prime}$ Cytogenetics, King Edward Memorial Hospital, Perth, Australia

${ }^{2}$ Derby Hospital, Derby, Australia

A peripheral blood sample was received on a 3-month-old female showing features of sclerocornea, failure to thrive and developmental delay. Peripheral blood chromosome analysis revealed an unbalanced three-way translocation between chromosomes 5,11 and 18 . There appeared to be a loss of a large amount of generic material on the chromosome 11 between bands q15 and q21. FISH studies confirmed there was a deletion at $11 \mathrm{q} 21$ and microarray studies demonstrated a $21 \mathrm{mb}$ deletion between 11q14.1 and 11q22.3. There is a limited number of molecularly characterized reports of similar deletions in this region of chromosome 11. These appear to be associated with a widely varying phenotype, from an apparently completely normal phenotype through to severe phenotypic abnormality. We compare the phenotype and cytomolecular characterisation of our case with other characterized cases in the literature. There is evidence that the 11q14-11q22 region contains an unusually large segment with haplosufficiency as well as a more proximal segment that is associated with exudative retinopathy. 


\section{PSYCHOSOCIAL AND GENETIC COUNSELING ISSUES IN PRENATAL DIAGNOSIS FOR SERIOUS GENETIC CONDITIONS AT UNIVERSITY MALAYA MEDICAL CENTRE

\author{
J. M.H. Lee', M. K. Thong' ${ }^{2}$ P. C. Tan \\ Malaysia \\ ${ }^{2}$ Department of Paediatrics, Faculty of Medicine, University Malaya, Malaysia \\ ${ }^{3}$ Department of Obstetrics and Gynaecology, Faculty of Medicine, University Malaya, \\ Malaysia
} \\ Department of Medical Social Work, University Malaya Medical Centre, Kuala Lumpur,}

Introduction: Chorionic villous sampling (CVS) is a relatively new technique of prenatal diagnosis testing performed in Malaysia. Psychosocial and genetic counseling issues are important considerations of prenatal diagnosis that require further studies in Malaysia. Purpose of Study: To determine the psychosocial issues experienced by the couple opting for prenatal diagnosis for serious or life-threatening genetic conditions, excluding chromosomal disorders and thalassaemia. Method: We identified 6 CVS that were performed for serious genetic disorders. The diagnoses were confirmed in the affected individuals in the families. Genetic counseling was provided and the pathogenic mutation was identified if parents opt to have another child and wish to proceed with prenatal diagnosis. Once the diagnosis is confirmed, the couples were re-counseled. A referral is made to the feto-maternal medicine unit team for counseling and CVS. The chorionic villi sample obtained is sent to laboratories accredited for DNA diagnosis. When the results are obtained, the couples are contacted for results disclosure and post-test counseling. Main Findings: The six CVS procedures were performed for congenital disorder of glycosylation (CDG) Type Ia (two pregnancies for one couple), myotonic dystrophy (two affected sisters in one family), ornithine transcarbamylase (OTC) deficiency (one) and spinal muscular atrophy (one). The psychosocial issues identified during pre-test counseling were the clients' difficulty in understanding complex genetic information, concerns on the CVS procedure and termination of pregnancy (TOP), high cost for testing and the limited time to arrange for CVS. Post-test counseling issues included the anxiety while waiting for results, uncertainty in decisionmaking, limited support after decision was made and guilt issues. Summary: The management of prenatal diagnosis for serious genetic conditions requires a multidisciplinary team effort to ensure the families can make informed choices regarding their reproductive options. Genetic counseling and identification of psychosocial issues are crucial to ensure an acceptable outcome for these families.

\section{CASE STUDY: HERLITZ JUNCTIONAL EPIDERMOLYSIS BULLOSA. GENETIC COUNSELING CHALLENGES IN A HIGHLY CONSANGUINEOUS NEW MIGRANT FAMILY}

\section{K. LeMarquand', J. Jonasson², A. Colley \\ ${ }^{\prime}$ Clinical Genetics, Liverpool Hospital, Sydney, Australia \\ ${ }^{2}$ Clinical Genetics, Laboratory Medicine, University Hospital of Umea, Umea, Sweden}

This case study involves a large Iraqi family with multiple consanguineous loops with a devastating family history of Herlitz junctional epidermolysis bullosa (H-JEB). H-JEB is a rare autosomal recessive skin disorder, usually fatal in infancy. It is characterized by skin fragility and blistering, nail inflammation; granulated wounds particularly on the face and a hoarse cry due to laryngeal involvement. The defective protein is laminin 332, a key component of the junction of the epidermis and dermis. Mutations in four genes have been described in JEB with the LAMB3 mutation accounting for $70 \%$ of all cases, including this family. The family believes at least 30 babies have died with this condition in the past 100 years and until 2008 the diagnosis was not known. The psychosocial issues in counseling this large extended family were complex and challenging. All communication was with an Arabic interpreter. Interviews with the women in this family revealed they: feared for every pregnancy, grieved for previous losses, experienced guilt from the perception of passing on faulty genes, had their religious beliefs challenged, and struggled to make reproductive choices A Swedish branch of the family was the first to have mutational analysis and following this Dr Jonasson and associ- ates in Sweden provided mutation testing on the Australian branches of the family. The mutation was found in the LAMB3 gene in exon6 (c.430C $>$ T) introducing a stop codon p.R144X. Five branches of the family in Australia have now had mutational analysis for carrier diagnosis and reproductive options.

\section{A CASE OF AML WITH MINIMAL DIFFERENTIATION} IN A WERNER SYNDROME PATIENT

\section{T. Lillis', H. Buyck', J.W. Dixon', G.White', C. Felix', R. Parfitt'}

${ }^{l}$ Central and Southern Regional Genetic Services, Wellington Hospital, Wellington, New Zealand

${ }^{2}$ Haematology, Wellington Hospital, Wellington, New Zealand

${ }^{3}$ I mmunology, Wellington Hospital, Wellington, New Zealand

Acute myeloid leukaemia ( AML) with minimal differentiation (WHO ICD-O code: 9872/3) accounts for less than 5\% of AML cases. There are no specific chromosomal aberrations associated with AML with minimal differentiation, however complex karyotypes with loss of $5 q$ have been reported. Werner syndrome (WS) is a rare autosomal recessive condition associated with premature aging, cataracts, diabetes mellitus, osteoporosis, atherosclerosis and leg ulcers. Neoplasia is frequently reported in these patients, most commonly soft tissue sarcomas and meningiomas. Few cases of hematological disorders have been reported in WS patients. This case describes a female patient with WS who developed AML with minimal differentiation at 55 years of age. The clinical manifestations of WS in this patient include scleroderma, severe lower leg ulceration, bilateral cataracts and atherosclerosis. The acquired cytogenetic findings associated with AML seen include a complex karyotype with loss of 5q, 17p and 12p and amplification of MYC through double minutes.

\section{CONGENITAL INSENSITIVITY TO PAIN DUE TO COMPOUND HETEROZYGOUS SCN9A MUTATIONS}

A. S. Ma,A. M.Turner

Medical Genetics, Sydney Children's Hospital, Sydney, Australia

Congenital insensitivity to pain (CIP) is a rare condition with fewer than 30 cases reported. MY is a 2-year-old boy with recurrent lower limb fractures complicated by osteomyelitis and non-union, and multiple accidental injuries. He was initially referred by the child protection team. This case highlights the difficulties involved in diagnosis and management of this condition. Pain is an important safety mechanism, and the absence of pain raises many health risks. Patients with CIP often have accidental burns, scalds, fractures, neuropathic joints, and can die early as a result of accidental trauma. MY has been found to be compound heterozygous for an SCN9A truncating mutation. SCN9A is a $\mathrm{Na}_{\mathrm{v}} 1.7$ channel involved in one of seven known alpha-subunits of the sodium channel. It is highly expressed in dorsal root ganglia and sensory neurons. Recessive loss-of-function truncating mutations are causative of CIP. This leads to pure pain insensitivity, and hypo/anosmia as the only symptoms. Patients with SCN9A mutations have normal neurological examination, intelligence, nerve conduction studies, MRI-brain, and nerve biopsy results. Interestingly, heterozygous gain-of-function mutations in one SCN9A allele cause two disorders: inherited erythromelalgia and paroxysmal extreme pain disorder. These are disorders where extreme pain and vascular flushing occur in response to stimuli such as exercise, heat, cold, or spicy foods. Heterozygous SCN9A mutations have also been implicated in channelopathy-associated seizure disorders such as Dravet syndrome. The main differentials to CIP include central causes and Hereditary Sensory and Autonomic Neuropathy (HSAN). 'Asymbolia' to pain is due to cortical damage in pain-processing centres of the cingulate or insular cortex. There are five subtypes of HSAN, each with their own mode of inheritance, genetic locus, and mixture of sensory, motor and autonomic involvement. 


\section{UNDERSTANDING, RISK PERCEPTION AND PSYCHOLOGICAL OUTCOMES THREE MONTHS AFTER PREDICTIVE GENE TESTING FOR HYPERTROPHIC CARDIOMYOPATHY AND LONG OT SYNDROME}

I. Macciocca ${ }^{1,2,7}$, O. Ukoumunne ${ }^{2,7}$, A. Davis ${ }^{2,3,7}$, R. Weintraub ${ }^{2,3,7}$, V. Connell ${ }^{3}$,

J. Ingles ${ }^{4,6}$, M. Delatycki ${ }^{2,7,12}$, S. Wake ${ }^{1,7}$, C. Semsarian ${ }^{4,5,11}$, L. Yeates ${ }^{4,5}$,

W. McKenna ${ }^{8,9}$,T. Marteau ${ }^{8}$, I.Winship ${ }^{7.13}$,V. Collins ${ }^{2}$

Genetic Health Services Victoria, Melbourne, Australia

Murdoch Childrens Research Institute, Melbourne, Australia

${ }^{3}$ Royal Children's Hospital, Melbourne, Australia

${ }^{4}$ Centenary Institute, Sydney, Australia

${ }^{5}$ Royal Prince Alfred Hospital, Melbourne, Australia

Royal Brisbane and Women's Hospital, Brisbane, Australia

University of Melbourne, Australia

Kings College London, London, Kingdom

${ }^{9}$ University College London, United Kingdom

${ }^{10}$ The Heart Hospital, London, United Kingdom

${ }^{\prime}$ University of Sydney, Australia

${ }^{2}$ Austin and Repatriation Hospital, Melbourne, Australia

${ }^{3}$ Royal Melbourne Hospital, Melbourne, Australia

Background: Hypertrophic cardiomyopathy (HCM) and Long QT syndrome (LQTS) are inherited cardiovascular conditions for which predictive testing has become more common. The most concerning feature of these conditions is sudden death which can be prevented if those at risk are identified. Methods: We conducted a multi-centre prospective questionnaire-based study to examine the impact of predictive genetic testing for HCM/LQTS. Feelings about testing, understanding, risk perception and psychological impact of the receiving a test result were examined. Participants $(n=77,14-67$ years old, 29 (37.6\%) tested positive) were recruited from four Australian and one British site. Questionnaires were completed before testing and at 2 weeks and 3 months post-disclosure. Results: Only one participant regretted being tested and one could not accurately recall their result. The majority of participants were pleased they had the test. Perceptions of the likelihood of developing disease and level of worry were consistent with gene test result. Multivariable regression analysis adjusting for baseline scores demonstrated a higher mean anxiety $(p<.001)$ and distress $(p<$ $.001)$ score in gene positive compared to gene negative participants at 2 weeks, but these differences were less apparent at 3 months. There was no difference in depression scores at any time point. Results did not change significantly after adjusting for potential confounders in the regression analysis. Conclusion: Participants were pleased to have undergone testing, understood the implications of their result and seemed to cope well psychologically.

\section{NARROWING THE 5031 DELETED REGION}

\section{IN MYELODYSPLASTIC SYNDROMES}

R. MacKinnon', M.Wall', G. Kannourakis', L. J. Campbell, ${ }^{1,3}$

${ }^{I}$ Victorian Cancer Cytogenetics Service, St Vincent's Hospital, Melbourne, Australia

${ }^{2}$ Ballarat Haematology and Oncology Services, Ballarat, Australia

${ }^{3}$ Department of Medicine (St Vincent's), University of Melbourne, Australia

Deletion of band $5 \mathrm{q} 31$ from the long arm of chromosome 5 (5q) is a recurrent abnormality in myeloid malignancies, presumed to remove a tumour suppressor gene (TSG). The TSG at 5q31 is still unknown. Deletion of a distinct region, 5q33, defines the 5q- syndrome, a category of myelodysplastic syndrome, and the TSG RPS14 has been identified (Ebert et al, 2008). To identify a TSG, a common deleted region (CDR) is first defined across a series of patients who have the deletion, to identify a set of candidate genes. Deletions are variable and only large deletions are detectable cytogenetically. Two published $5 \mathrm{q} 31 \mathrm{CDRs}$ have a combined length of $2.5 \mathrm{Mb}$ containing 29 genes. We present a case with a cryptic deletion of $5 \mathrm{q} 31.2$ which reduces the CDR. In a patient diagnosed initially with a myelodysplastic syndrome the only chromosome abnormality identified by karyotyping was a 20q deletion. On progression to acute myeloid leukaemia there was no change in karyotype. Using array comparative genomic hybridisation, which can detect microscopically invisible deletions, we detected a $1.3 \mathrm{Mb} 5 \mathrm{q}$ deletion which was also shown to be present at diagnosis. Had this second abnormality been detected by cytogenetics at diagnosis the patient's disease would have been classified as intermediate rather than good prognosis. Together with the previously published CDRs this case reduces the CDR to $108 \mathrm{~kb}$ containing four candidate TSGs: the preferred candidate $E G R I$ (epidermal growth factor re ceptor 1), JMJDIB (Jumonji domain-containing protein $1 \mathrm{~B}), R E E P 2$ (Receptor expressionenhancing protein 2) and ETF1 (Eukaryotic peptide chain release factor subunit 1). Identification of the TSG at $5 \mathrm{q} 31$ will pave the way for the development of targeted therapies to treat malignancies with this deletion.

\section{ANALYSIS OF NUCLEIC ACID EXTRACTED FROM FIXED CHROMOSOME SUSPENSIONS AND OLD REFRIGERATED BONE MARROW SPECIMENS \\ R. MacKinnon', C. Selan², H. Nandurkar ${ }^{3,4}$, L. J. Campbell ${ }^{1,3}$ \\ ${ }^{I}$ Victorian Cancer Cytogenetics Service, St Vincent's Hospital, Melbourne, Australia \\ ${ }^{2}$ Immunology Research Centre, St Vincent's Hospital, Melbourne, Australia \\ ${ }^{3}$ Department of Medicine (St Vincent's Hospital), University of Melbourne, Australia \\ ${ }^{4}$ Department of Haematology, St Vincent's Hospital, Melbourne, Australia}

The analysis of DNA and RNA from archival specimens is useful for identifying the critical genetic changes in a variety of disorders. However these approaches are limited by the absence of suitable tissue samples, particularly for retrospective analysis of hematological malignancy. We investigated the suitability of DNA prepared from (a) bone marrow samples (referred for karyotyping in hematological malignancies) refrigerated for up to 36 days before processing, and (b) fixed cytogenetic specimens, for array comparative genomic hybridisation (array $\mathrm{CGH}$ ) and single nucleotide polymorphism (SNP) array. We also attempted Real-Time PCR (RTPCR) with RNA extracted from fixed cytogenetic preparations. These specimens are not usually considered as sources of total RNA or genomic DNA as it is assumed that these nucleic acids are too degraded. Cytogenetic suspensions were rinsed and resuspended in PBS before processing. DNA was extracted using a DNeasy Cell and Tissue Kit (Qiagen,). Array CGH (Agilent,) and SNP array (Illumina,) were carried out according to the manufacturers' protocols. DNA from chromosome preparations was slightly degraded but most was longer than $18 \mathrm{~kb}$. DNA from 36 day-old bone marrow was the most degraded at $4-20 \mathrm{~kb}$. Microarray results were comparable to those obtained using fresh bone marrow. Total RNA was purified from chromosome samples using the RNeasy Mini Kit (Qiagen). Assessment of our RNA samples on an Agilent Bioanalyser showed RNA concentrations between 1.5 to $13 \mathrm{ng} / \mathrm{ul}$ with all samples being fairly degraded. Despite the level of RNA degradation seen, we were able to perform RT-PCR analysis and obtain meaningful gene expression data from all samples. The use of DNA and RNA from fixed cytogenetic preparations makes a wider range of archival material available for retrospective study.

\section{'IT'S BETTER TO KNOW THAN NOT KNOW' FIRST-HAND ACCOUNTS OF PREDICTIVE GENETIC TESTING IN ADOLESCENTS FOR ADULT ONSET CONDITIONS}

C. Mand ${ }^{1,2}$, L. Gillam ${ }^{1,3}$, R. E. Duncan', M. B. Delatycki' ${ }^{1,4}$

${ }^{1}$ Murdoch Childrens Research Institute, Melbourne, Australia

${ }^{2}$ Department of Medicine, Monash University, Australia

${ }^{3}$ School of population health, University of Melbourne, Australia

${ }^{4}$ Austin Health, Melbourne, Australia

Background: Asymptomatic individuals at-risk of genetic conditions can undergo predictive testing (PT) to determine if they will develop the condition later in life. PT is routinely offered to adults at-risk of late-onset conditions (e.g., Huntington disease). More controversial is PT in minors for adult-onset conditions where no effective treatment or prevention exists. The ethical discourse concerning PT in minors is lively, yet it remains one of speculation, with a relative scarcity of empirical evidence. Aim: To explore young people's experience of being tested for an adult-onset condition for which no medical intervention exists. Methods: Semi-structured interviews 
with eight young people who underwent PT for adult-onset conditions as minors ( 6 gene-positive, 2 gene-negative). Interviews were transcribed and thematically analyzed. Results: Interviews revealed a range of harms and benefits associated with the PT experience. Harms were not limited to individuals who received a gene-positive result. Participants identified potential harms with being refused a test as well as the testing process; however these were outweighed by the benefits that arose. Benefits were described by all participants, regardless of result. Participants experienced difficultly living with uncertainty prior to testing, and all described feelings of relief when receiving their test result. Irrespective of the result, no participant regretted being tested. Conclusions: These data add to the breadth and complexity of the discourse about PT in minors, highlighting factors which have been previously under-represented in the literature. Current approaches towards counseling and other processes prior to PT may need to be re-considered in light of the negative experiences of the testing process reported by some participants. For the first time this study gives voice to the adolescents who are the centre of this debate, inviting reconsideration of whether the current guidelines have reached the correct balance in terms of pros and cons of PT in minors.

\section{FIRST QUEENSLAND PATIENT WITH HETEROZYGOUS MUTATION IN ABCB4 PRESENTING WITH FIBROSING CHOLESTATIC LIVER DISEASE}

\author{
C. S. Manning, J. McGaughran, L. Powell \\ Clinical Genetics, Royal Brisbane and Womans Hospital, Brisbane, Australia
}

$A B C B 4$ mutations are responsible for a spectrum of cholestatic liver diseases starting in neonatal life or in late adulthood and including progressive familial intrahepatic cholestasis type 3 (PFIC3), low phospholipid-associated cholelithiasis, and intrahepatic cholestasis of pregnancy. $A B C B 4$ translocates the major biliary phospholipid, phosphatidylcholine (multidrug resistant protein 3 - MDR3), from the inner to the outer leaflet of the canalicular membrane. More recently more subtle forms of MDR3 protein deficiency with later onset have been shown to cause of progressive intrahepatic fibrosis and cirrhosis. These adult onset forms are commonly associated with heterozygous mutations in $A B C B 4$ whilst the more severe juvenile onset forms such as PFIC3 result from homozygous mutations. This case is the first Queensland report of a patient with a heterozygous mutation of $A B C B 4$. A 51-year-old lady presented with deranged liver function tests initially presumed to be autoimmune hepatitis. However low antibody levels coupled with a lack of a response to immunosuppression led to the diagnosis being revisited. Immunostaining for MDR3 protein was abnormal and coupled to her response to ursodeoxycholic acid led to consideration of $A B C B 4$ mutation as a cause. Her past history included intense pruritus with both of her pregnancies. Her mother had identical symptoms with her pregnancies. This is the first reported Queensland case of a heterozygous $A B C B 4$ mutation causing progressive intrahepatic fibrosis, and also illustrates the milder phenotypic spectrum of MDR3 protein loss. Adult physicians should consider the possibility of $A B C B 4$ mutations in patients presenting with unexplained progressive intrahepatic fibrosis responsive to ursodeoxycholic acid and refer to genetic service for consideration of cascade testing of relatives.

\section{MALE RECIPROCAL TRANSLOCATION CARRIERS - FREQUENCY OF THE DIFFERENT MEIOTIC SEGREGATION PATTERNS INFLUENCES THE LIKELIHOOD OF A SUCCESSFUL PREGNANCY}

N. J. Martin, S.A. Grehan, J. Irving

Cytogenetics, Queensland Fertility Group, Brisbane, Australia

Males with a low sperm count are known to have a greater risk of carrying a constitutional chromosome abnormality - these include numerical sex chromosome changes, reciprocal and Robertsonian translocations as well as inversions, insertions and ESACs. However, not all males presenting with infertility have a low sperm count as an indicator of their carrier status for a chromosome rearrangement, and would thus not be routinely karyotyped. At QFG the policy is to karyotype all couples at part of their infertility investigations. Couples where the male is a translocation carrier frequently opt for PGD as part of the IVF process to ensure only chromosomally balanced or normal embryos are transferred. Not all such couples achieve success with IVF. We have investigated 10 male reciprocal translocation carriers with FISH probes, such that each chromosome in the translocation is uniquely identified. A minimum of 500 sperm were scored for the meiotic segregation patterns for each patient (sperm count permitting). The frequency of normal or balanced sperm ranged from 23 to $44 \%$. The number of IVF cycles undertaken ranged from $2-10$. In only $40 \%$ of cases (4/10) was an embryo transfer (ET) or live birth achieved. In these cases the frequency of normal or balanced sperm was greater than $35 \%$ (range $37.5-43 \%$ ). In cases where no ET or live birth was achieved, there was a higher frequency of tertiary and interchange monosomy (12.5-18.7\% vs. $5.2-7.8 \%$ ). Sperm FISH meiotic segregation patterns constitute a useful tool in male reciprocal translocation carriers considering assisted reproductive technologies (ART). It allows the detection of couples who are unlikely to achieve a pregnancy (naturally or by ART) and allows counseling and consideration of the use of donor sperm to achieve a successful pregnancy.

\section{CARDIAC GENETICS: THE FIRST 250 CLINIC PATIENTS \\ J. McGaughran', L. Hunt', J.Atherton ${ }^{2}$ \\ ${ }^{\prime}$ Genetic Health Queensland, Human Genetics Society of Australasia, Brisbane, Australia \\ ${ }^{2}$ Royal Brisbane and Women's Hospital, Department of Cardiology, Brisbane, Australia}

In February 2007, a monthly cardiac genetics clinic was commenced at the Royal Brisbane and Women's Hospital. The clinic was to ensure appropriate counseling, genetic testing and management of those with or at risk of an inherited cardiac condition. A range of conditions have been seen including; hypertrophic cardiomyopathy, familial dilated cardiomyopathy, arrythmogenic right ventricular cardiomyopathy, catecholaminergic polymorphic ventricular tachycardia and long QT syndrome. Rarer conditions such a Fabry disease and Danon disease have also been seen. To date 250 patients have been seen through the clinic. Because of pressure on clinic space, a number of the patients referred to the genetic service have been seen in genetics clinics at locations around the state. A number of challenges have been encountered: management of those with a mutation but no phenotype; testing of at risk children; funding for testing; waiting times with increasing numbers of cases referred. The clinic also collaborates with the forensic pathology service and coroner in the assessment of those families where there has been a sudden unexplained death in a young individual. The data from the clinic will be presented.

\section{THE CHALLENGES OF ARRAY CGH; \\ A TALE OF THREE PATIENTS}

\section{J. McGaughran', G. Peters ${ }^{2}$}

'Genetic Health Queensland, Human Genetics Society of Australasia, Brisbane, QLD, Australia

${ }^{2}$ Cytogenetics, Westmead, Human Genetics Society of Australasia, Sydney, Australia

Since the introduction of the routine clinical use of aCGH in the diagnosis of patients with a range of conditions, a number of challenges have emerged. Some aCGH findings may be associated with both a normal and abnormal phenotype. In others the significance is uncertain and testing of other family members is needed to clarify the significance of the change found. Three cases will be presented that illustrate some of these challenges. Case 1: Misleading aCGH result A patient with short stature, dysmorphism and a cardiac conduction abnormality presented to clinic. Her mother had the same phenotype. She had an aCGH which demonstrated an abnormal result. Both this and the subsequent diagnosis will be presented. Case 2: Inherited from parent A patient had autism and microcephaly. aCGH showed a duplication of $16 \mathrm{p} 13.11 \mathrm{p} 13.11$ The father also had the same duplication and was phenotypically normal. Case 3 : Not inherited from parent. 
The female proband had delayed development, marked hypotonia and dysmorphic features. Her mother had a similar phenotype. aCGH showed a deletion of 17p11.2 and a duplication of 3p26.1. Her mother had a normal aCGH.

\section{SINGLE NUCLEOTIDE POLYMORPHISM (SNP)- BASED ARRAY COMPLEMENTS CLASSICAL CYTOGENETICS IN DETECTION OF CHROMOSOMAL ABERRATIONS IN WILMS TUMOR}

\section{R. Md Zin', K. Pham²,A. Murch', A. Charles'}

${ }^{\prime}$ Pathology, School of Pathology and Laboratory Medicine, University of Western Australia, Australia

${ }^{2}$ Australian Neuromuscular Research Institute, Perth, Australia

Background: Wilms tumors have characteristic chromosomal abnormalities such as $11 \mathrm{p} 13$ deletion in a subset of tumors. Reports comparing chromosomal imbalances detected by SNP array and conventional karyotype in Wilms tumor have been very few. Objective: To compare conventional karyotypes of Wilms tumor with SNP array findings of the same samples. Materials and Methodology: DNA extraction was performed on 47 frozen tumor samples with preexisting conventional cytogenetics results. A total of 43 tumor specimens were successfully arrayed with Affymetrix Cytogenetics Whole-genome $2.7 \mathrm{M}$ array. Four tumors either had severely degraded DNA or failed the passing yield of SNP values. Conventional karyotype results were available for 38 tumor specimens. Results: Correlation between SNP array and conventional karyotype findings were possible in 38 specimens. The SNP array and classical cytogenetics results were concordant in $87 \%$ (33 of 38 specimens) and were either completely discordant or indeterminate in $13 \%$ ( 5 of 38 specimens). Tumor with normal karyotypes had LOH/UPD regions and cryptic abnormalities on SNP array. SNP array confirmed unbalanced and balanced translocations seen by karyotype in some tumors. SNP array was able to support the findings of trisomies, tetrasomies and large deletions detected by classical cytogenetics. In addition, SNP array was also able to detect regions of LOH/UPD in $41 \%$ of Wilms tumor specimens. Conclusion: Our results show that both techniques are able to complement each other. SNP array does not only validate the lesions detected by conventional chromosomal banding, but also identified additional gains and losses as well as regions of UPD. Discrepancies could be explained by inherent limitations of each technique, inadequate tumor sampling and tumor heterogeneity.

\section{AN ONLINE SCREENING DECISION AID FOR MEN WITH A FAMILY HISTORY OF PROSTATE CANCER: DEVELOPMENT AND PILOT-TESTING}

B. Meiser ${ }^{1,2}$, K.Watts ${ }^{1,2}$, C. E.Wakefield ${ }^{3,4}$, U. Sansom-Daly ${ }^{4,5}$, A. Barratt ${ }^{6}$,

M. I. Patel' , E. A. Lobb ${ }^{8}$, C. L. Gaff, 10,11, K. Howard ${ }^{6}$, G. J. Mann ${ }^{13}$

${ }^{I}$ Medical Oncology, Prince of Wales Hospital, Sydney, Australia

${ }^{2}$ Prince of Wales Clinical School, University of New South Wales, Australia

${ }^{3}$ School of Women's and Children's Health, University of New South Wales, Australia

${ }^{4}$ Centre for Children's Cancer and Blood Disorders, Sydney Children's Hospital, Sydney, Australia

${ }^{5}$ School of Psychology, University of New South Wales, Australia

${ }^{6}$ School of Public Health, University of Sydney, Australia

${ }^{7}$ Department of Surgery, University of Sydney, Australia

${ }^{8}$ Calvary Health Care, Sydney, Australia

${ }^{9}$ WA Centre for Cancer \& Palliative Care, Curtin University of Technology and Edith

Cowan University, Perth, Australia

${ }^{10}$ Genetic Health Services Victoria, Melbourne, Australia

${ }^{\prime \prime}$ Murdoch Children's Research Institute, Melbourne, Australia

${ }_{12}^{12}$ Paediatrics and Medicine, University of Melbourne, Australia

${ }^{13}$ Westmead Institute for Cancer Research, University of Sydney at Westmead Millennium Institute, Australia

Background: Men with a family history of prostate cancer have unmet information needs relating to how best to manage their cancer risk. This study aimed to develop and pilot test an online decision aid (DA) tailored to men with a family history of prostate cancer to assist them to make an informed choice about screening. Methods: Men aged 40-79 years with a family history of prostate cancer were invited to participate in the study by a letter conveyed to them by an affected male relative who attended a urology outpatient clinic. The DA was evaluated by eligible men in two stages. First, men evaluated the acceptability of a paper-based version of the DA in a questionnaire $(N=22)$. Second, the same men were asked to appraise the functionality of an online version of the DA and to provide their feedback in a telephone interview $(N=20)$. Results: Ninety-one percent of participants reported that they found the paper-based DA useful, and that it contained enough information to make a decision about screening (73\%). All men reported that the online DA was easy to use, with the majority reporting that they would recommend the website to other men. Most men also reported that they would prefer to receive information about screening via a website rather than in a booklet (78\%). Conclusions: This study provides a valuable insight into the optimal design and distribution of information about prostate cancer screening for men who are at increased risk of this disease.

\section{ATTITUDES AND INFORMATION NEEDS OF WOMEN NEWLY DIAGNOSED WITH OVARIAN CANCER REGARDING TREATMENT-FOCUSED GENETIC TESTING}

B. Meiser ${ }^{1,2}$, M. Gleeson ${ }^{1,3}$, N. Kasparian ${ }^{4}$, K. Barlow-Stewart ${ }^{5}$, M. Ryan ${ }^{6}$,

G. Mitchell', A. Trainer ${ }^{7}$, M. Friedlander', K. Tucker'

${ }^{I}$ Medical Oncology, Prince of Wales Hospital, Sydney, Australia

${ }^{2}$ Prince of Wales Clinical School, University of New South Wales, Australia

${ }^{3}$ Hunter Genetics, Newcastle, Australia

${ }^{4}$ School of Women's and Children's Health, University of New South Wales, Australia

${ }^{5}$ Centre for Genetics Education, Sydney, Australia

${ }^{6}$ Royal Hospital for Women, Sydney, Australia

${ }^{7}$ Familial Cancer Service, Peter McCallum Cancer Centre, Melbourne, Australia

Background: Until recently, there seemed little practical value for a woman with ovarian cancer in knowing her BRCA mutation status, other than the potential benefits for unaffected family members. However, there is growing evidence that in the near future BRCA mutation status may be used to make treatment recommendations, and that women with ovarian cancer will be referred for genetic counseling and testing shortly after diagnosis (treatment-focused genetic testing - TFGT). This qualitative study aimed to assess women's attitudes toward TFGT and to identify their information preferences about TFGT. Methods: Women $(N=22)$ with ovarian cancer who had either (1) advanced disease and had previously had TFGT $(N=12)$ or $(2)$ had a recent ovarian cancer diagnosis and were asked about their hypothetical views of TFGT $(N=10)$, were interviewed in-depth. Results: All participants reported being willing to undergo TFGT if it had the potential for a targeted therapy. Most women wanted to be informed about TFGT prior to their surgery for ovarian cancer. The primary motivation for TFGT was more treatment options and improved survival. Assisting family with risk information was another significant motivation. Slightly more women preferred to receive the information from their medical oncologist, compared to a genetics specialist. The preferred format for the educational material was brief written information. Conclusion: Acceptability for TFGT is high, with many wanting it to be routinely offered to all women newly diagnosed with ovarian cancer. Brief written educational materials need to be developed to support women in making informed decisions about TFGT. 


\section{ACCEPTANCE, EXPERIENCES AND INFORMATION PREFERENCES OF YOUNG WOMEN NEWLY DIAGNOSED WITH BREAST CANCER REGARDING TREATMENT-FOCUSED GENETIC TESTING}

B. Meiser ${ }^{1,2}$, M. Gleeson ${ }^{3}$, K.Watts ${ }^{1,2}$, J. Kirk ${ }^{4}$, C. Saunders ${ }^{5}$, K. Barlow-Stewart ${ }^{6}$, E.A. Lobb ${ }^{7}, \mathrm{G}$. Mitchell ${ }^{8}, \mathrm{~K}$. Tucker

${ }^{\prime}$ Medical Oncology, Prince of Wales Hospital, Sydney, Australia

${ }_{2}^{2}$ Prince of Wales Clinical School, University of New South Wales, Australia

${ }^{3}$ Hunter Genetics, Newcastle, Australia

${ }^{4}$ Familial Cancer Service, Westmead Hospital, Sydney, Australia

${ }^{5}$ School of Surgery, University of Western Australia, Australia

${ }^{6}$ Centre for Genetics Education, Sydney, Australia

${ }^{7}$ Calvary Health Care, Sydney, Australia

${ }^{8}$ Familial Cancer Service, Peter McCallum Cancer Centre, Melbourne, Australia

Background. An increasing number of women newly diagnosed with breast cancer and with a family history of breast/ovarian cancer or with other features indicative of a high mutation carrier risk are being offered genetic testing to guide their treatment (treatmentfocused genetic testing - TFGT). This qualitative study aimed to identify young women's attitudes; prior experiences if any; and information preferences regarding TFGT. Methods. Women $(N=26)$ with breast cancer $(<50$ yrs $)$ who had either (1) previously had TFGT $(N=14)$ or $(2)$ had a recent breast cancer diagnosis and were asked about their hypothetical views of TFGT $(N=12)$ participated in semi-structured interviews. Results: TFGT was highly acceptable. Most women wanted to be informed about it at or around the time of their cancer diagnosis. The primary motivation for TFGT was to obtain information to inform appropriate surgical decisions. Assisting family with risk information was another significant motivation, in addition to assisting with decision-making about reproduction and breast reconstruction. Half of the women preferred to receive the information from a genetics specialist, genetic counsellor or their breast care nurse, while half preferred to receive information from their surgeon or medical oncologist. The preferred format for the educational materials was brief written information. Conclusion: TFGT is highly acceptable to many women, and they want to be informed about the testing around the time of their breast cancer diagnosis. We are currently in the process of developing and pilot-testing a brief pamphlet on TFGT.

\section{'LIFE ON THE FRONT LINE': EXPERIENCES OF HEALTHCARE PROFESSIONALS WORKING WITH FETAL ANOMALY}

\section{M.A. Menezes ${ }^{1,2}$, J. M. Hodgson 1,2, M. Sahhar ${ }^{2,3}$, S.A. Metcalfe ${ }^{1,2}$ \\ ${ }^{\prime}$ Genetics Education and Health Research, Murdoch Childrens Research Institute, Melbourne, Australia \\ ${ }^{2}$ Department of Paediatrics, The University of Melbourne, Australia \\ ${ }^{3}$ Genetic Health Services Victoria, Melbourne, Australia}

Each year in Victoria, Australia 4\% of pregnancies will be diagnosed with a fetal anomaly. Healthcare professionals working in fetal medicine settings are often involved in the diagnosis and/or counseling of women at risk of a fetal anomaly. They often see patients who are in crisis situations triggered by the potential loss of a pregnancy, resulting in strong emotional reactions. This crisis state can cause patients to 'shut down' making it difficult for healthcare professionals to engage them in decision making around whether or not to continue the pregnancy. Decision-making around termination of pregnancy can raise ethical, moral and legal issues not only for patients but also for healthcare professionals and can lead to feelings of stress. The aim of this study was to explore the experiences of healthcare professionals who work with fetal anomaly and to identify any supports used/needed. 40 in-depth interviews were conducted with medical and allied healthcare professionals who work with couples receiving a diagnosis of a fetal anomaly. Interviews were analyzed using thematic analysis. Participants discussed a range of challenging situations they encounter due to the nature of their work. Breaking 'bad' news was identified as one of the most difficult aspects of this setting and participants discussed at length the emotional nature of working with patients in crisis. All participants reported that working with fetal anomaly had an impact on their daily lives, particularly when they were pregnant themselves. Most participants spoke about feeling largely unsupported in their work and felt that these impacts resulted in 'burnout' and high staff turnover. Working with fetal anomaly appears to have a substantial impact on healthcare professionals. This impact needs to be acknowledged and additional training and supports should be put in place, particularly around 'self-care' and breaking 'bad' news.

\section{INFANTILE SIALIC ACID STORAGE DISEASE - A RAPID DIAGNOSIS BY MSMS}

A. Mishra', M. Eggington', M. Lipke', H. Peters', P. Clements' ${ }^{2}$,. Pitt ${ }^{2}$

'Metabolic Screening Laboratory, VCGS Pathology, Melbourne, Australia

${ }^{2}$ Department of Biochemical and Community Genetics, Biochemical Genetics, Adelaide, Australia

Infantile free sialic acid storage disease (ISSD) is a recessively inherited lysosomal storage disorder caused by impaired transport of free sialic acid from lysosomes, and the resultant accumulation of sialic acid in tissues. ISSD is characterized by severe developmental delay, coarse facial features, hepatosplenomegaly and cardiomegaly; death usually occurs in early childhood. We report a male infant of non-consanguineous Eritrean parents presenting antenatally with non-immune hydrops fetalis. There was a history of recurrent miscarriage. Hydrops was diagnosed at 21 weeks gestation and required in utero ascetic tap. Generalized edema, large anterior fontanel, hepatosplenomegaly and bilateral talipes equino varus were noted following vaginal delivery at 36 weeks gestation. Cranial ultrasound showed lenticulostriate calcification. The clinical course was complicated by patent ductus arteriosis, sepsis, urinary tract infection and pneumonia culminating in progressive respiratory compromise related to gross abdominal distension and he died at 37 days of age. A lysosomal storage disorder was strongly suspected. A urine sample collected on day 4 of life was screened by negative ion electrospray MSMS and showed markedly increased free sialic acid. Two subsequent samples confirmed this gross increase and quantitation using a colourimetric assay was $1600 \mathrm{mmol} / \mathrm{mmol}$ creatinine (RR $10-80 \mathrm{mmol} / \mathrm{mmol}$ ). The diagnosis of ISSD was confirmed by the finding of increased sialic acid content in cultured skin fibroblasts: $105 \mathrm{nmol} / \mathrm{mg}$ cell protein (RR 0-10 nmol/mg). Screening for sialic acid was readily incorporated into an existing urine screening program for amino acids and organic acids using MSMS. In this case, MSMS provided a rapid confirmatory diagnosis within the first week of life.

\section{IDENTIFICATION OF NOVEL DISEASE GENES IN ANTERIOR EYE DISORDERS}

S. Mohamed Yousoof ${ }^{3}$, L. St Heaps², A. Darmanian², G. Peters'², R. Jamieson

Eye Genetics Research Group, Children's Medical Research Institute

and Save Sight Institute, Sydney, Australia

${ }^{2}$ Cytogenetics, The Children's Hopsital at Westmead, Sydney, Australia

${ }^{3}$ Sydney Medical School, The University of Sydney, Sydney, Australia

The development of the human eye depends on highly differentiated tissues that result from a proper interaction between several genes encoding transcriptional factors and signaling molecules. Disruption of the genes can cause abnormal eye structures that can critically affect the ocular function and blockage of aqueous humor flow, thus increasing the risk of developing glaucoma. In some cases, the structural anomalies can lead to severe visual impairment and leave the patients with few treatment options. Some genes including PITX2 and FOXC1, are known to be associated with eye anterior segment dysgenesis and glaucoma. In the majority of patients, the underlying disease genes are not yet known. In the present study, we apply high resolution array $\mathrm{CGH}$ and characterization of chromosomal translocations to identify novel candidate disease genes in patients with anterior segment disorders/glaucoma. We identified two families with chromosome abnormality regions. Using bioinformatic analyses, we have identified candidate disease regions. These regions contain novel transcription factor and signaling pathway molecules in developmental eye disease. These findings highlight the utility of 
CGH microarray and chromosomal anomaly analysis in candidate novel disease gene identification.

\section{QUANTIFICATION OF TRANSGENE EXPRESSION FROM LENTIVIRAL VECTORS CONTAINING THE F2A SEQUENCE IN THE EXPRESSION CASSETTE}

L. Mortimer', S. Brice',Y. Irani', H. Brereton', M. Keane', P. Cowan², D. Coster', K.Williams'

Ophthalmology, Flinders University, Australia

Immunology Research Centre, St. Vincent's Hospital, Melbourne, Australia

The use of multigenic lentiviral vectors for gene therapy to transfer more than one transgene targeting more than one pathway has potential for treating complex conditions such as corneal allograft rejection. Our aims were to compare the level of transgene expression from single-gene and double-gene lentiviral vectors. Double-transgene vectors were constructed by inserting the F2A self-processing sequence between two transgenes in the vector expression cassette, permitting expression of two transgenes within a single open reading frame. Analogous single-gene vectors were also constructed. Transgenes included cDNAs encoding intracellular enhanced yellow fluorescent protein (eYFP) and secreted proteins anti-rat $\mathrm{CD} 4$ antibody fragment (anti-CD4 $\mathrm{scFv}$ ) and endostatinkringle5 fusion protein (EK5). The expression of the transgenes cloned into the double-gene vectors, each transgene upstream and downstream of the F2A sequence, was compared to expression from their single-gene counterparts by transfecting HEK293A and CHO cells. Anti-CD4 $\mathrm{scFv}$ protein was quantified by flow cytometry on rat thymocytes, EK5 by ELISA, and eYFP by flow cytometry. Protein expression from the double-transgene vectors was significantly lower than from their corresponding single-transgene counterparts for eYFP and anti-CD4 scFv. Further, expression of transgenic proteins targeted to different cellular compartments (eYFP and anti-CD4 scFv) was reduced when a transgene was positioned downstream of F2A, compared with upstream $(\mathrm{p}<0.05)$. Comparable levels of transgenic protein expression were observed from double-gene vectors encoding transgenic proteins targeted to the same cellular compartment (anti-CD4 $\mathrm{scFv}$ and EK5), irrespective of the transgene order in the string. In summary, levels of transgenic protein expression targeted to different cellular compartments were significantly reduced when the transgene was positioned downstream of F2A in a double-gene vector, but were comparable when proteins were targeted to the same compartment. Expression of transgenic protein from a double-gene vector was significantly reduced compared with its single-gene counterpart.

\section{A COMPARISON OF MOLECULAR AND CYTOGENETIC} TECHNIQUES FOR THE DIAGNOSIS OF PREGNANCY LOSS

M. Caramins', T. Saville', R. Shakeshaft', G. L. Mullan', B. Miller', M.Y.Yip', M. F. Buckley ${ }^{2}$

${ }^{I}$ Molecular Genetics, SEALS, Prince Of Wales Hospital, Sydney, Australia

${ }^{2}$ Dept Human Genetics, University Nijmegen Medical Centre, Nijmegen, Netherlands

Purpose: To evaluate the sensitivity, specificity, advantages and limitations of multiplex ligation-dependent amplification (MLPA) compared with conventional karyotype analysis in the investigation of contributing factors to recurrent pregnancy loss (RPL). Method: A cohort of 284 patients underwent side-by-side analysis of products of conception (POC) by both conventional karyotyping and multiplex ligation-dependent probe amplification (MLPA) with direct comparison of results. Results: MLPA was shown to enable a diagnosis for an additional $47(16.5 \%)$ patients compared with conventional karyotype analysis. However, this advantage was offset by some disadvantages of the method, including a high false positive rate $(8 / 104 ; 7.7 \%)$, as demonstrated by single arm probe abnormalities of uncertain clinical significance, as well as the inability to characterise structural rearrangements, such as Robertsonian translocations, which comprised $2.46 \%$ of samples (99\% CI $=0.09-4.83$ ), and ploidy changes. The calculated performance characteristics of MLPA in this cohort yielded a sensitivity of $86.9 \%$, and specificity of $92.4 \%$. Conclusions: The advantages of now widely-accepted molecular methodologies, such as lower failure rates, faster turnaround times and lower cost, must be complemented by adequate counseling, family follow-up and specific diagnostic reporting practices. Although MLPA shows acceptable test characteristics for use in this setting, it is particularly important to specifically address the limitations of this methodology, including the inability to characterise structural rearrangements and ploidy changes if MLPA is to be performed alone.

\section{PHYSICAL AND PSYCHOLOGICAL DILEMMA IN LATE IDENTIFIED CONGENITAL ADRENAL HYPERPLASIA (CAH)}

A. Ediati',2, M. Muniroh', B.A. Setyawati ${ }^{3}$, A. Z. Juniarto', S. M.H. Faradz', F. H. De Jong ${ }^{4}$,A. B. Dessens ${ }^{4}$, S. L.S. Drop ${ }^{4}$

${ }^{\prime}$ Center for Biomedical Research, Faculty of Medicine, Diponegoro University, Semarang, Indonesia

${ }^{2}$ Faculty of Psychology, Diponegoro University, Semarang, Indonesia ${ }^{3}$ Master Programme of Genetic Counseling, Faculty of Medicine, Diponegoro Universi, Semarang, Indonesia

${ }^{4}$ Erasmus Medical Center, Rotterdam, Netherlands

Simple virilizing $\mathrm{CAH}$ is an autosomal recessive disorder characterized by 21 hydroxylase deficiency leading a.o. to excessive adrenal androgen production. In patients with $46 \mathrm{XX}$ karyotype, the androgen overproduction will lead to a gradual virilisation of the external genital. Patients will be born with an ambiguous genital hindering gender assignment. In case there is no treatment, genital virilisation will be progressive. We present two 46, XX CAH patients aged 17 and 33 at diagnosis. Hormonal values, and CYP21 gene mutation analysis confirmed the diagnosis in.both patients. Physical manifestation revealed severely masculinized genital genitalia (Prader stages 4 and 3) and masculine secondary sex characteristics in the adult females. Patient A had been assigned female at birth. She gradually changed gender and presented himself as a male at the first visit. Psychological assessment indicated he had developed a male gender identity male gender role behavior and felt sexually attracted to females (measurements: Indonesian versions of Hines' Gender Questionnaire, Hines' Activity questionnaire and Kinsey scale). He decided to continue his life as male. Subsequently hydrocortisone treatment was stopped and recommendation for surgical phallus reconstruction was given. Patient B entered our clinic at age 33. She had been assigned and raised female and identified herself as a female. She felt sexually attracted to males. She started Prednisone treatment at age 33 and menses started thereafter. She underwent clitororeduction. Her male appearance first restrained her to engage in romantic relationships but at age 38 she got married. The complex management of adults with CAH calls for attention to raise awareness among medical personnel in order to promote early detection and treatment of $\mathrm{CAH}$ patients. Keywords: $\mathrm{CAH}$, late identification, gender assignment, Indonesia.

\section{PRENATAL DIAGNOSIS OF A SKELETAL DYSPLASIA-GENETIC AND COUNSELING CHALLENGES}

K. R. Murphy

Prenatal Genetics, Sydney Ultrasound for Women, Sydney, Australia

A 30-year-old woman (G1P0) with no significant family history for herself or partner presented for her 19 week morphology ultrasound at Sydney Ultrasound for Women. Nuchal translucency and first trimester biochemistry [NTplus] assessment at 13 weeks was low risk at which time fetal measurements were consistent with menstrual dates. At the 19-week morphology scan shortened long bones $(<10$ th centile) were identified as well as angulation in both the right and left tibia. The diagnosis of a skeletal dysplasia was discussed with the couple, specifically Osteogenesis Imperfecta [OI] and Hypophosphotasia. Amniocentesis revealed a normal female karyotype $(46 \mathrm{XX})$. The couple elected to terminate the pregnancy just prior to 20 weeks. Autopsy revealed abnormal bone density and mineralization. Subsequently DNA from the fetus and the parents were sent for testing in France, which confirmed the diagnosis of Hypophosphatasia. These results took almost 1 year to complete. 
This case illustrates the challenges faced in prenatal genetic counseling where a diagnosis is not immediately available. Time restrictions on termination of pregnancy necessitate a decision be made based on the information available at the time, without a firm diagnosis available for either condition. The couple had to act on the information that was available from the ultrasounds and the information provided on OI and Hypophosphatasia during counseling. As the majority of patients have little to no knowledge of genetics or genetic testing, this case highlights the challenges of working with a couple in emotional distress, helping them to understand the effects of two different conditions, why a diagnosis cannot be made quickly and how it can take a long time for the diagnosis to be confirmed.

\section{THE UPTAKE OF PREDICTIVE TESTING IN LONG QT SYNDROME AND HYPERTROPHIC CARDIOMYOPATHY}

J. Nagy ${ }^{1,2,3}$, E. Muggli ${ }^{2}$, S.Wake ${ }^{1,2,3}$, P. James ${ }^{3}$, O. Ukoumunne ${ }^{2}$, I. Macciocca ${ }^{2,3}$

${ }^{I}$ Department of Paediatrics, University of Melbourne, Australia

${ }^{2}$ Murdoch Children's Research Institute, Melbourne, Australia

${ }^{3}$ Genetic Health Services Victoria, Melbourne, Australia

Hypertrophic Cardiomyopathy (HCM) and Long QT Syndrome (LQTS) are two cardiac genetic conditions associated with sudden cardiac death. After the detection of a pathogenic mutation in a family, cascade predictive testing can be offered to family members. The aims of this research study were to determine the uptake of predictive testing for LQTS and HCM, to evaluate predictors of uptake, and to compare the rates of uptake of testing for HCM and LQTS to other conditions where predictive testing is available. A retrospective audit of genetic files was performed to collect the data from Victorian families where a mutation known to cause either HCM or LQTS had been identified prior to 1 June 2009, and who had attended our service for genetic counseling in the period 1 January 2006 and 1 September 2009. Data was collated in a custom built database and analyzed using STATA statistical software. Overall, the uptake of predictive testing for first and second degree relatives of the proband in the families analyzed was found to be $32 \%$. Families with LQTS had a higher rate of uptake (37\%), than the families with a history of HCM (23\%). Predictors of uptake identified in this study included: being a first degree relative, having a family history of sudden death and being female (HCM only). The uptake of predictive testing for LQTS was similar to that found in studies on hereditary breast cancer but higher than that observed in studies on Huntington's disease, while the uptake of predictive testing for HCM was lower than that observed for hereditary breast cancer and similar to that found in Huntington's disease. This study makes recommendations for future research.

\section{EVALUATION OF AN EDUCATION DAY FOR CAREGIVERS OF ADULTS WITH PHENYLKETONURIA}

\section{Netting', E. Clover', I. Chapman², D. Bratkovic ${ }^{3}$}

${ }^{\prime}$ Department of Clinical Dietetics, Royal Adelaide Hospital, Adelaide, Australia ${ }^{2}$ Division of Medicine, University of Adelaide, Australia

${ }^{3}$ Metabolic Unit, Children, Youth and Women's Health Service, Adelaide, Australia

Background: The Metabolic Clinic at the Royal Adelaide Hospital is responsible for the management of eleven adults with late diagnosed Phenylketonuria (PKU) in whom a low protein diet and phenylalanine free supplement is beneficial. Ten of these adults live in supported accommodation. Individuals attend metabolic clinic for follow up with a caregiver, and may not be accompanied by the same staff members at each attendance. This makes continuing education of caregivers regarding management of the diet for PKU difficult. Aim: The aim of the seminar was to provide a forum to teach caregivers of adults with PKU about the dietary management and monitoring of PKU, and to demonstrate use of basic low protein products for cooking. This paper reviews the attendance and evaluation of the seminar. Method: Staff working with adults with PKU on diet and supplement were invited to attend a three hour seminar about the management of PKU. Nutricia provided funding for the venue and a home economist skilled in low protein cooking.
Knowledge was assessed by a multiple choice 'PKU Quiz' at the beginning and end of the session, and the quality of the morning was evaluated by a questionnaire at the end of the session. Results: The seminar was attended by a total of seventeen people representing four different organisations, and one adult with PKU. Thirteen completed evaluation questionnaires and 'PKU Quizzes' were received. There was an increase in total score from an average of $6 / 10$ to $8 / 10$ in the PKU knowledge quiz. Overall evaluation of the seminar was positive. Conclusion: This was the first seminar in South Australia targeted at caregivers of adults with PKU. We have been able to demonstrate an increase in knowledge regarding management of PKU amongst the caregivers who attended the seminar and plan to repeat these seminars biannually.

\section{DIAGNOSIS OF AUTOSOMAL RECESSIVE GENETIC DISORDERS USING SNP MICROARRAY ANALYSIS - TWO CASE STUDIES}

C. Ngo, D. Bruno, H. Slater, T. Burgess

Cytogenetics, VCGS Pathology, Melbourne, Australia

Conventional cytogenetic testing by microscopy detects mutations which act dominantly to cause phenotypic abnormality. The recent use of array CGH and SNP arrays for chromosome analysis has demonstrated detection of mutations that manifest through a wide range of aetiologies. We describe two examples of 'recessive mutations' detected using Affymetrix SNP 6.0 and Cyto 2.7M arrays. Both cases involved long continuous stretches of homozygosity (LCSH) which harboured a deletion. The LCSH is believed to have arisen either by uniparental isodisomy or by chance through 'ancestral identity by descent' or through consanguinity. In the first case, the individual presented with congenital ichthyosis and developmental delay, and was clinically diagnosed with Sjogren-Larsson syndrome. On analysis with SNP microarray, a $5.5 \mathrm{~kb}$ homozygous deletion was detected involving the 3 ' end of the ALDH3A2 gene at $17 \mathrm{p} 11.2$. This finding confirmed the clinical diagnosis for this individual. In the second case, the clinical features included feeding abnormalities, laryngomalacia, global developmental delay, dysmorphism, and hypotonia. The SNP microarray analysis showed a $161 \mathrm{~kb}$ intragenic homozygous deletion of the VPS13B gene at $8 \mathrm{q} 22.2$, which is consistent with a clinical diagnosis of Cohen syndrome. These 2 cases demonstrate the potential of array analysis to detect recessive disorders arising from homozygous deletions. It also yields candidate genes where there is no established genotype-phenotype relationship.

\section{MOUSE MODELS OF DOMINANT ACTA1 DISEASE RECAPITULATE HUMAN DISEASE AND PROVIDE INSIGHT INTO THERAPIES}

G. Ravenscroft', C. Jackaman', S. Brignans ${ }^{3}$, J. Papadimitriou ${ }^{4}$ L. M. Griffiths ${ }^{5}$, E. McNamara', A. J. Bakker', , K. E. Davies', N. G. Laing', K. J. Nowak'

${ }^{\prime}$ Centre for Medical Research, University of Western Australia, Australia

${ }^{2}$ Physiology, University of Western Australia, Australia

${ }^{3}$ Proteomics International, Nedlands, Perth, Australia

${ }^{4}$ School of Pathology and Laboratory Medicine, University of Western Australia,

Australia

${ }^{5}$ Neuropathology, Royal Perth Hospital, Perth, Australia

${ }^{6}$ Department of Physiology, Anatomy and Genetics, University of Oxford, Oxford,

United Kingdom

Mutations in the skeletal muscle $\alpha$-actin gene (ACTAl) cause a range of pathologically defined congenital myopathies. Most patients have dominant mutations and experience severe skeletal muscle weakness, dying within one year of birth. To determine mutant ACTA1 pathobiology, transgenic mice expressing ACTA1(D286G) were created. These $\operatorname{Tg}(A C T A 1) \mathrm{D} 286 \mathrm{G}$ mice were less active than wildtype individuals. Their skeletal muscles were significantly weaker by in vitro analyses and showed various pathological lesions reminiscent of human patients, however they had a normal lifespan. Mass spectrometry revealed skeletal muscles from $\operatorname{Tg}(A C T A 1) \mathrm{D} 286 \mathrm{G}$ mice contained $\sim 25 \%$ ACTA1(D286G) protein. Tg(ACTAl)D286G mice were crossed with hemizygous Actal $^{+/-}$knock-out mice to generate $\operatorname{Tg}(A C T A 1) \mathrm{D} 286 \mathrm{G}^{++}$. Actal $^{+/-}$offspring: homozygous for the 
transgene and hemizygous for the endogenous skeletal muscle actin gene. Akin to most human patients, skeletal muscles from these offspring contained approximately equal proportions of ACTA1(D286G) and wild-type actin. Strikingly, the majority of these mice presented with severe immobility between postnatal days 8 to 17 , requiring euthanasia. Their skeletal muscles contained extensive structural abnormalities as identified in severely affected human patients, including nemaline bodies, actin accumulations and widespread sarcomeric disarray. Therefore we have created valuable mouse models, one of mild dominant ACTAl disease $(\operatorname{Tg}(A C T A 1) \mathrm{D} 286 \mathrm{G})$, and the other of severe disease, with a dramatically shortened lifespan $\left(\operatorname{Tg}(A C T A 1) \mathrm{D} 286 \mathrm{G}^{+/+}\right.$. Acta $\left.^{+/-}\right)$. The correlation between mutant ACTA1 protein load and disease severity parallels effects in ACTA1 families and suggests altering this ratio in patient muscle may be a therapy for patients with dominant ACTAl disease. Furthermore, pathological changes characteristic of the myofibrillar myopathies as well as ringbinden were observed in these mouse models. The presence of such features suggest that patients with myofibrillar myopathy and/or ringbinden of unknown genetic origin should be considered for ACTAl mutation screening.

\section{AFFYMETRIX CYTOGENETICS WHOLE-GENOME 2.7M ARRAY TESTING IN A COMMUNITY COHORT WITH INTELLECTUAL DISABILITY}

\footnotetext{
E. Palmer', H. Spiers' ${ }^{2}$, G. Turner ${ }^{3,4}$, S. Einfeld ${ }^{5}$, B. Tonge ${ }^{6}$, D. Mowat ${ }^{1,3}$

${ }^{1}$ Clinical Genetics, Sydney Children's Hospital, Sydney, Australia

${ }^{2}$ Ramaciotti Centre for Gene Function Analysis, University of NSW, Australia

${ }^{3}$ School of Women's and Children's Health, University of NSW, Australia

${ }^{4}$ Hunter Genetics, Hunter Area Health Service, Newcastle, Australia

${ }^{5}$ University of Newcastle, Australia

${ }^{6}$ Centre for Developmental Psychiatry, Monash University, Australia
}

The Australian Child to Adult Development (ACAD) Study is a community (urban, semi-urban, rural) based longitudinal study of psychopathology in 429 young people with intellectual disability from the states of NSW and Victoria in Australia (Tonge et al 1996, Partington et al 2002). Approximately half of the patients remained undiagnosed after clinical and baseline genetic investigations. This subgroup was categorized into 5 clinically descriptive groups (dysmorphic, 28\%; non-dysmorphic 37\%; autism, 12\%; cerebral palsy, $13 \%$; and severe seizure disorder, $10 \%$ ). Consent was obtained for 95 individuals to undergo sub-microscopic copy number testing. The first tier of testing detected a sub-telomeric aberration by MLPA in 9 $\%$, and a likely pathogenic aberration in $11 \%$ of tested patients using Affymetrix 6.0 and $250 \mathrm{~K}$ arrays. The second tier of testing used the new ultra-high resolution Affymetrix 2.7M Oligo and SNP array to analyse the remaining patients $(n=69)$. Results were analyzed using the Affymetrix ChAS software. Copy number variants (CNVs) were coded as (1) likely to be pathogenic, (2) variants of uncertain clinical significance or (3) likely to be benign, by systematic comparison with databases of benign (DGV) and pathogenic (DECIPHER) CNV, RefSeq and OMIM genes. CNV characteristics of the different clinical subgroups were compared. A scoring system was devised to classify the variants of uncertain clinical significance based on suggested guidelines by Lee et al, 2007 and the CNV were run through the GeCCO scoring software (Hehir-Kwa et al, 2010). The sample was also analyzed for mosaicism and areas of loss of heterozygosity. This study both assesses the effective diagnostic yield of applying an ultra-high resolution aCGH to a community cohort of patients with intellectual disability, as well as highlighting some of the practical and interpretation difficulties.

\section{EXPLORING PEDIATRIC GENETIC CONSULTATIONS: IMPLICATIONS FOR TRAINING}

J. Paul ${ }^{1,2,3}$, J. Hodgson ${ }^{1,2}$, L. Stirling ${ }^{3}$, S. Metcalfe ${ }^{1,2}$

${ }^{I}$ Genetics Education and Health Research, Murdoch Childrens Research Institute, Melbourne, Australia

${ }^{2}$ Department of Paediatrics, The University of Melbourne, Australia

${ }^{3}$ School of Languages and Linguistics, The University of Melbourne, Australia

Developmental delay (DD) is estimated to affect $1-3 \%$ of the population and is a common reason for children to be referred to genetic clinics. The underlying cause of DD often remains unknown with some studies reporting that $80 \%$ of patients with severe DD have no known etiology. For parents, receiving a diagnosis can be a source of validation, and may impact upon their child's prognosis, treatment and access to available supports. A core role of the clinical geneticist is to explain complex and abstract genetics at an appropriate level in order to facilitate parent's understanding of their child's condition and available tests. Such tests may include molecular karyotyping, which is now being offered to children with undiagnosed DD. To date there has been limited research into the process of paediatric genetic consultations. We conducted a systematic review of process studies in genetics consultations, identifying 47 papers. Only 4 involved pediatric settings. Studies found that patients' and clinicians' agendas were sometimes mismatched and demonstrated how communication patterns can influence decision-making around childhood testing. A variety of aims and methodological approaches were used making it difficult to assess the most appropriate method for analyzing these interactions. Although these studies offer some insights into communication processes in genetics settings, they are of limited value as most failed to explore perspectives of both clinicians and patients. Based on a critical review of existing literature and informed by consultations with key stakeholders and clinic observations, we have developed an appropriate and relevant methodology using a qualitative approach. This will enable us to capture the process and explore parent and clinician experiences and perspectives of pediatric genetics consultations. We anticipate that findings from the proposed study will inform best practice in this area of medical communication.

\section{TREATMENT OF A METHYLMALONIC ACIDURIA MOUSE MODEL WITH INTRA-LIVER TRANSPLANTATION OF A DEVELOPED NOVEL LIVER PROGENITOR CELL LINE}

H. Peters' ${ }^{2}$, N. Buck², F.Ahmad Hafad², J. Pitt', G. Yeoh ${ }^{3}$

${ }^{\prime}$ Metabolic Unit, Genetic Health Services Victoria, Melbourne, Australia

${ }^{2}$ Cell and Gene Therapy Unit, MCRI, Melbourne, Australia

${ }^{3}$ School of Biomedical, Biomolecular and Chemical Sciences, The University of Western Australia, Australia

Aim: We aim to investigate the use of intra-liver transplantation of a developed liver progenitor cell line for the treatment of methylmalonic aciduria (MMA) using a mouse model with an intermediate phenotype of MMA. Introduction: Methylmalonic aciduria (MMA) is an organic acid disorders resulting from a functional defect in the mitochondrial enzyme methylmalonyl-CoA mutase. We have developed a mouse model that recapitulates the key aspects of MMA: elevated methylmalonic acid levels in urine, blood and various organs. These mice are significantly smaller in size and thus there is a phenotype in addition to biochemical markers for determining disease correction. Methods: Mouse pups were intra-liver injected with 0.2 million cells at 1 and 4 days old (double transplant). Several groups were set up: untransplanted, sham (phosphate-buffered saline) and F-EGFP cell lines. These cells are an immature liver progenitor cell line established by the 'plate and wait' method from congenic embryonic day 14 fetal liver cells. Each group contained at least 3 litters of pups. Mice were monitored for 16 weeks before analysis. Results: Three of the 23 mice in the study had detectable cell engraftment and repopulation. Increased body weight and decreased blood $\mathrm{C}_{3}$ carnitine levels, plasma MMA levels and urine MMA levels were observed in proportion of cell-transplanted MMA mice indicating potential disease correction by the transplanted cells. 
Conclusions: This study concludes that intra-liver transplantation into mouse pups provides a promising therapy as biochemical and phenotypic improvement were observed in MMA cell-transplanted mice. Although the percentage of EGFP DNA was very low, this indicated that the cells were dividing and functional in the liver. Disease correction demonstrated in this study suggests a great opportunity in the clinical field and further investigations into this possibility are warranted.

\section{CASE REPORT: A PATIENT WITH DEL 22011.2 PRESENTING WITH POLYARTERITIS NODOSA}

G. Poke, M. Gabbett, S. Kanagarajah, R. Shakhovskoy

Royal Brisbane and Womens Hospital, Melbourne, Australia

22q11.2 deletion syndrome is associated with both immunodeficiency and autoimmunity. It has been estimated that up to $10 \%$ of people with a 22q11.2 deletion develop some form of autoimmune disease. There are a number of theories as to why this may occur, including increased infections, impaired regulatory $\mathrm{T}$ cells and impaired thymic central tolerance. Previously reported phenomena in people with 22q11.2 deletion include autoimmune cytopenias, autoimmune thyroid disease, juvenile arthritis, vitiligo, type 1 diabetes mellitus, antiphospholipid syndrome and inflammatory bowel disease. This case is the first report of a patient with both 22q11.2 deletion syndrome and vasculitis. A 32-year-old lady presented with a seizure and was found to have stage II hypertension and acute kidney injury. Renal biopsy confirmed a necrotizing arteritis. She also had a sensorimotor axonal neuropathy causing a painful peripheral neuropathy, consistent with polyarteritis nodosa. She had been diagnosed with esophageal candidiasis a week earlier. Her only past history included a small ventricular septal defect, mild intellectual impairment and psoriasis. Besides being the first reported case of vasculitis in 22q11.2 deletion syndrome, this case illustrates the lifetime risk of immune dysregulation in this syndrome. Adult physicians should consider the possibility of undiagnosed $22 \mathrm{q} 11.2$ deletion syndrome in patients presenting with unexplained immunodeficiency or autoimmunity.

\section{TRANSLATING EVIDENCE BASED RESEARCH INTO A TOOL FOR CONSUMERS}

\section{L.A. Purser, K. Barlow-Stewart \\ Centre for Genetics Education, New South Wales Department of Health, Sydney, Australia}

Given the rapid progress in the field of genetics, the complexity of the information and its consequences for families and the media surrounding it, information needs for consumers are high. The challenge is to ensure the community has access to current and appropriate information that ensures they are well informed and in a good position for decision-making. The Centre for Genetics Education has established an evidence-based process for the development of Fact Sheets, brochures and booklets as well as e-formatted and web based information. The Centre systematically follows the steps: (1) Identification of key resources needed through findings in the literature, current research conducted in partnership with clinical services, expert request, consumer request, media issues, and audit of resources informed by the Centre's database; (2) Literature review to establish new content; (3) Research: collaborative studies to guide direction, language and priority; (4) Consumer advice and expert consultation; (5) Content writing and design guided by the Centre's research and NHMRC guidelines; (6) Consumer and expert reviews pre-publication. The Centre distributes and provides access to a wide number of resources: For example in the twelve months June 2009-June 2010, the CGE website received 12,000 visits per week, distributed Prenatal testing: Special tests for your baby during pregnancy booklet (6,140 copies) and Screening Tests for Your Baby in Early Pregnancy brochure (9,648 copies). New resources developed in 2010, in both print and e-format, include Huntington Disease and Genetic Testing Booklet (March 2010); Breast and Ovarian Cancer that Runs in the Family,
Information and Decision Aid (August 2010), Lynch Syndrome and Genetic testing, Information and Decision Aid (August 2010), Micro-Array (Fact Sheet) and update of 60 fact sheets and over 80 information Sheets.

\section{QUALITY OF LIFE AND MRI CHANGES IN HEMIZYGOTE MALE AND HETEROZYGOTE FEMALE PATIENTS WITH FABRY DISEASE}

\section{H. Quinn, M. C.Tchan, D. O. Sillence}

Department of Genetic Medicine, Westmead Hospital, Sydney, Australia

Fabry disease is an X-linked inherited lysosomal storage disorder due to deficiency in a-galactosidase (a-GAL A). This has protean consequences including cerebrovascular manifestations. There are also quality of life disturbances due to living with this chronic illness and its manifestations, which include severe pain crises and symptoms across all organ systems. The present study aimed to assess these quality of life issues by analyzing short form-36 (SF-36) data from 32 patients with Fabry disease (18 male hemizygotes, 14 female heterozygotes) in an Australian cohort. Males with Fabry disease scored significantly lower than the normal population in the SF-36 domains of: (1) role limitations due to physical functioning (moderate effect size), (2) vitality (moderate effect size), (3) general health perceptions (large effect size) and (4) physical component summary (large effect size). Females with Fabry disease scored significantly lower than the normal population for the domains of: (1) general health perceptions (moderate effect size) and (2) vitality (large effect size). Intracranial MRIs were assessed for 22 patients ( 16 male, 6 female). Abnormal MRIs were recorded in 12 male and 3 female patients. The rate of abnormal MRI findings was significantly greater in male patients affected by Fabry disease compared to female patients $\left(\mathrm{t}_{30}=2.76, p<.05\right)$. Those with abnormal MRIs were significantly older than those with normal imaging findings $\left(\mathrm{t}_{30}\right.$ $=3.28, p<0.01)$. No significant differences were found in SF-36 scores when comparing those patients with normal and abnormal MRI findings. This study confirms that, in the previously unstudied NSW cohort of patients, female heterozygotes are not merely carriers of Fabry disease but also suffer significant quality of life disturbances and cerebrovascular manifestations, although to a lesser extent than their male counterparts.

\section{CHALLENGES FOR ADOLESCENT PATIENTS: \\ THE EXPERIENCES OF YOUNG PEOPLE WITH IMPLANTABLE CARDIOVERTER DEFIBRILLATORS}

B. Rahman', R. E. Duncan ${ }^{1,3}$, I. Macciocca' ${ }^{1,4}$, S. Kamberi ${ }^{2,5}$, M. Sahhar ${ }^{1,4}$, V. Connell ${ }^{2,5}$

${ }^{\prime}$ Murdoch Childrens Research Institute, Melbourne, Australia

${ }^{2}$ Cardiology Department, The Royal Children's Hospital, Melbourne, Australia

${ }^{3}$ Centre for Adolescent Health, Melbourne, Australia

${ }^{4}$ Genetic Health Services Victoria, Melbourne, Australia

${ }^{5}$ Medtronic, Australia

An implantable cardioverter defibrillator (ICD) is a device used in the treatment of individuals with life-threatening cardiac conditions. These include genetic disorders such as long QT syndrome, hypertrophic cardiomyopathy and Brugada syndrome, all of which have the propensity to cause sudden cardiac death. The ICD detects abnormal heart rhythms and delivers a shock to the heart to restore a normal heart rhythm when necessary. Adult ICD patients consistently report elevated levels of anxiety and depression, as well as negative lifestyle changes associated with the device. With little data available about young people living with ICDs, the psychosocial impact of having the device as an adolescent is largely unknown. Knowledge of adolescents' attitudes to their ICD and medical management may better inform those involved in their care, as well as other clinicians working with adolescents. The current study aimed to explore the experiences of adolescents living with an ICD. Parents of these adolescents were also included to better understand the impact of receiving an ICD on the family as a whole. A qualitative approach was chosen to explore the lived experience; semistructured interviews with six adolescents and six parents were 
conducted from which a number of key themes emerged. Key themes to emerge from the data included the restrictions adolescents face post-ICD implantation and the limitations adolescents impose on themselves. Important findings also emerged relating to communication between health professionals, parents and adolescents, the ongoing challenges faced by adolescents and the disparity between parental and adolescent perceptions of 'being normal'. Despite the challenges both adolescents and parents were able to adjust to life after receiving an ICD and described the benefits of having the device. These findings have significant implications for clinical practice and may provide helpful information for those involved in the care of adolescents and their families.

\section{A NEW AUSTRALIAN CASE OF RAINE SYNDROME \\ S. Rajagopalan', B. Hanna', A. Adno 2 \\ ${ }^{\prime}$ Dept of Clinical Genetics, Liverpool Hospital, Sydney, Australia \\ ${ }^{2}$ Fetomaternal Unit, Liverpool Hospital, Sydney, Australia}

Raine syndrome is a rare autosomal recessive osteosclerotic skeletal dysplasia associated with narrow thorax, distinctive craniofacial dysmorphic features and brain abnormalities including intracranial calcification. In 2007, mutations in the FAM20C gene were identified to be the cause of the condition. Raine syndrome appears to be neonatal lethal in the majority of cases, mainly due to respiratory failure from thoracic hypoplasia. Only 19 cases from 15 families have been described in the literature, and there is a high incidence of parental consanguinity. There is only one previously published case in Australia in 1992. We present a female infant with a clinical diagnosis of Raine syndrome, who died shortly after birth due to profound respiratory distress. She was the offspring of consanguineous (first cousin) parents. Antenatal ultrasound scans revealed polyhydramnios, in-utero growth retardation, lemon-shaped head, protuberant eyes, severe micrognathia and diffusely increased echogenicity of the periventricular white matter. Examination of the infant soon after death revealed the characteristic dysmorphic features of Raine syndrome. The salient features included microbrachycephaly, wide fontanelles, marked proptosis and micrognathia, severely hypoplastic nose, carp-like mouth, midline gingival cleft, bell-shaped chest and telebrachyphalangy. Diagnostic findings on skeletal survey included diffuse osteosclerosis, subperiosteal new bone formation, pseudofractures of the ribs and striking ossification of the base of the skull and facial bones. MRI brain scan suggested the presence of multiple intracerebral calcifications. The parents declined an autopsy. Mutation analysis of the FAM20C gene has been arranged to confirm the diagnosis. This case highlights that the features of Raine syndrome are so distinctive that a non-invasive post-mortem diagnosis can be made with a careful clinical assessment, brain imaging (preferably CT) and a skeletal survey.

\section{SHAKING THE BRANCHES OF THE FAMILY TREE: EXPLORING THE IMPACT OF FAMILY HISTORY SCREENING IN PRIMARY CARE}

\section{G. Reid', F.Walter', J. Emery' \\ General Practice, University of Western Australia, Australia \\ General Practice \& Primary Care Research Unit, University of Cambridge, United Kingdom}

Background: Whilst an adverse risk assessment could potentially harm family relationships, the benefits to patients surrounding the identification of increased familial risk of chronic disease, intentions and motivation to improve lifestyle and screening habits, as well as the perceived value of universal family history screening in primary care warrants exploration. Aim: To assess the impact of family history screening in primary care using qualitative in-depth interviewing techniques. Methods: A sub-group of participants from an over-arching, larger Family History Screening Study were invited to take part in telephone interview. Purposive sampling was undertaken of those who had been shown to have an increased familial risk of heart disease, diabetes or cancer, as well as those at 'population risk'. The in-depth interview was informed by a topic guide based on relevant literature and revised based on emerging findings from the iterative analytic process. Interviews were audio-taped; fully transcribed and analyzed both manually and via NVivo 8. Findings: Family history screening was overall viewed as a positive experience that allowed participants to explore an aspe ct of their health that they may not have otherwise done. Cancer was viewed as the most worrisome disease compared to heart disease and diabetes, which were viewed as being largely lifestyle determined. Family was a key motivating factor when making choices around lifestyle improvement and preventive screening. Conclusions: Identifying patients at increased familial risk of developing chronic disease is an essential aspect of preventive primary care. As well as the need for a validated tool to collect family history information, it is important that the impact of such screening be explored to minimise potential psychological harm.

\section{MICROARRAY: DISCOVERY OF AN ASYMPTOMATIC MALE WITH A DMD GENE MUTATION}

A. Ritchie', S. Corrie ${ }^{2}$,T. Burgess' ${ }^{2}$, S.White ${ }^{3}$, D. Du Sart'

${ }^{\prime}$ Molecular Genetics, VCGSPathology, Murdoch Childrens Research Institute, Melbourne, Australia

${ }^{2}$ Cytogenetics, VCGS, Murdoch Childrens Research Institute, Melbourne, Australia ${ }^{3}$ Clinical Services, Victorian Clinical Genetics Service Pathology, Murdoch Childrens Research Instit, Melbourne, Australia

The sample from a 3-day-old female baby was received by the VCGS Pathology Cytogenetics laboratory requesting FISH for a possible 22q11 deletion and karyotype. The baby had a complete atrioventricular septal defect (AVSD). Her sample was included in the routine microarray analysis and an incidental finding of a deletion in the DMD gene was identified on the short-arm of one $\mathrm{X}$ chromosome, indicating that she was a carrier of Duchenne Muscular Dystrophy (DMD) or Becker Muscular Dystropy (BMD). No other abnormalities were identified. The DMD gene deletion was confirmed using MLPA by the molecular genetics laboratory. The mother was tested using MLPA and found to be negative for any deletions in the DMD gene. As an unlikely outcome, the patient's father was also tested and shown to have the same deletion as his daughter! Mutations in the DMD gene causes a range of phenotypes in males, from severely affect DMD patient's presenting in childhood to mildly affected BMD patient's who generally present in the 2 nd decade of life. In addition, X-linked myalgia, an X-linked recessive disorder characterized by muscle cramps and myalgia, has been reported to have deletions in the DMD gene. The father in this case is 38 years old and is completely asymptomatic. Microarray analysis has brought to light a new situation. Previously only males showing symptoms of BMD and DMD were tested; however this case is more complicated given the only male tested in this family is asymptomatic. This introduces new complexities for clinicians and genetic counsellors when offering advice to families given the 'unknowns' involved in this case. This case therefore hi-lights that complete analysis of all family members should be considered in determining the significance of abnormal findings, regardless of the current knowledge of the genetics and clinical presentations of specific disorders.

\section{THE EFFECT ON FAMILIES OF HAVING A CHILD WITH A METABOLIC CONDITION}

\section{J. Robinson}

Department of Medical Genetics, Sydney Children's Hospital, Sydney, Australia

Inborn errors of metabolism (IEM) are chronic conditions that affect the whole family. While very rare individually, as a group their incidence is 1 in 500-1000 live births. The number of IEMs diagnosed is increasing in Australia due to the implementation of Newborn Screening programs. The treatment of these conditions usually requires medication and complex restricted diets. The diagnosis of an IEM in a child has a large impact on the family unit. The complex dietary restrictions can be difficult to implement and the frequent hospital admissions, monitoring of the child's condition, and time consuming treatment regime can all place stress on the family. The 
fact IEMs are inherited can add an extra layer of stress to the family in the form of guilt. There can be some positive impacts on family members such as increased tolerance and compassion, increased social skills and a more positive outlook on life. The presentation will explore the effects on the family unit, both positive and negative, of having a child with an IEM.

\section{$X$ MARKS THE SPOT UPDATE AND FUTURE DIRECTION FOR THE NSW GOLD SERVICE}

\author{
D. F. Ross', M. J. Field', A. Hackett', , M. Hunter', C. Rogers', J. Boyle', C. J.Turner', \\ L. Christie' \\ ${ }^{I}$ Genetics of Learning Disability Service, Hunter Genetics, Waratah, Australia \\ ${ }^{2}$ University of Newcastle, Australia
}

There are over 200 genes on the $\mathrm{X}$ chromosome that are thought to have a role in brain function and cognition. Identifying genes and causative mutations is challenging and often requires collaborations with international research groups. The Genetics of Learning Disability (GOLD) Service is a NSW state funded service providing genetic counseling to families with known or suspected X-Linked Mental Retardation (excluding Fragile $\mathrm{X}$ families). The major aims of the service are to identify a genetic cause for these family histories and offer reproductive options for at risk females in the families. We present our current testing algorithm, diagnostic rates by family category, with a diagnosis being achieved in more than $50 \%$ of families in some diagnostic groups, and scope for future testing using high through put technology.

\section{EXPLORATION OF THE CULTURAL CONTEXT OF CANCER, KINSHIP AND GENETICS AMONGST ARABIC- AUSTRALIANS: IMPLICATIONS FOR PRACTICE}

M. Saleh ${ }^{1,2}$, B. Meiser ${ }^{4,5}$, J. Kirk', K. Tucker ${ }^{6}$, M. Eisenbruch ${ }^{8}$, K. Barlow-Stewart ${ }^{1,3}$

${ }^{I}$ NSW Health, Centre for Genetics Education, Sydney, Australia

${ }^{2}$ Faculty of Medicine, The University of New South Wales, Australia

${ }^{3}$ Faculty of Medicine, The University of Sydney, Australia

${ }^{4}$ Psychosocial Research Group, Prince of Wales Hospital, Sydney, Australia

${ }^{5}$ Prince of Wales Clinical School, The University of New South Wales, Australia

${ }^{6}$ Hereditary Cancer Clinic, Prince of Wales Hospital, Sydney, Australia

${ }^{7}$ Familial Cancer Service, Westmead Hospital, Sydney, NSW, Australia

${ }^{8}$ School of Psychology and Psychiatry, Monash University, Australia

Objectives: To explore with Arabic-Australian patients' and their communities' culturally determined attitudes / beliefs about kinship, hereditary cancer and other illnesses, family health history and the impact on communication. Method: The design is an ethnographic and qualitative interview study with thematic analysis. Arabic-Australian participants were recruited in two stages. Firstly, patients who had attended a family cancer clinic were invited to participate. Secondly, participants from the general community were recruited with the aim of either verifying or challenging the reported findings from the clinic sample. Results: A total of 38 interviews with 26 females and 12 males provided cultural descriptions of illnesses, including cancer, and its causes. The terminologies around cancer and a cancer diagnosis were believed to have more impact and generate greater fear in people in the Arabic community living in Australia than the terms used to describe a diagnosis of other illnesses. Despite this, those interviewed were aware of screening and community programs and were open to discuss their views about cancer with the research team. Whilst respondents recognized family health history as an important factor that reflects on one's likelihood of successful child-bearing, models of kinship described were not in line with Western concepts. Levels of acculturation were not linked to beliefs in culture-specific traditions such as consanguinity; however lower levels of acculturation were related to beliefs in ensuring a healthy blood lineage in potential marriage partners. Conclusion: Arabic-Australians are an important group in the community with unique culture-specific attributes that affect communication in the clinical setting. Barriers such as the negative feelings associated with cancer and the stigma it arouses, together with a lack of awareness about inherited cancer, must be acknowledged. Culturally competent clinical practices with this group include the incorporation of these belief systems into the communication process and avoidance of stereotyping.

\section{PHARMACOLOGICAL SCREENING FOR THE THERAPY OF FRIEDREICH ATAXIA}

L. Li', S.Van Den Hengel', J. Cook', C. Sandi', M. Pook', P.A. loannou ${ }^{2,3}$,

M. B. Delatycki ${ }^{1,3}$, J. P. Sarsero ${ }^{1,2,3}$

'Bruce Lefroy Centre for Genetic Health Research, Murdoch Childrens Research Institute, Melbourne, Australia

${ }_{2}^{2}$ Cell and Gene Therapy Research Group, Murdoch Childrens Research Institute, Melbourne, Australia

${ }^{3}$ Department of Paediatrics, The University of Melbourne, Australia

${ }^{4}$ Hereditary Ataxia Research Group, Brunel University, Uxbridge, United Kingdom

Friedreich ataxia (FRDA) is an autosomal recessive disorder characterized by neurodegeneration and cardiomyopathy. The presence of a GAA trinucleotide repeat expansion in the first intron of the $F X N$ gene results in the inhibition of gene expression and an insufficiency of the mitochondrial protein, frataxin. There is a correlation between expansion length, the amount of residual frataxin and the severity of disease. As the coding sequence is unaltered, pharmacological upregulation of $F X N$ expression may restore frataxin to therapeutic levels in patients. To facilitate screening of compounds that modulate $F X N$ expression in a physiologically relevant manner, we have established a genomic reporter assay consisting of stable HeLa cells containing an $F X N-E G F P$ fusion construct (in-frame fusion of the $E G F P$ gene with the entire normal human genomic $F X N$ locus on a BAC clone). The cell line was used to establish a fluorometric cellular assay for use in high throughput screening (HTS) procedures. A small chemical library of primarily FDA-approved compounds and natural extracts has been screened and analyzed. Compound hits identified by HTS have been further evaluated by flow cytometry in the cellular genomic reporter assay. The effects on FXN mRNA and frataxin protein levels have been measured in cell lines derived from individuals with FRDA and in a GAA repeat expansion mouse model of FRDA. Any compound that specifically increases frataxin levels by several-fold in FRDA patients could serve as a potential pharmacological therapy for FRDA.

\section{TRPV4-RELATED SKELETAL DYSPLASIAS: A CLINICAL, RADIOGRAPHIC , AND MOLECULAR STUDY IN 19 NEW FAMILIES}

R. Savarirayan', E. Andreucci',2, J. F. Bateman ${ }^{3}$, S. Aftimos ${ }^{4}$, E.A. Haan ${ }^{5}$,W. Hunter ${ }^{6}$, B. Kerr ${ }^{7}$, R. Gardner', M. Patricelli', G. McGillivray', D. Sillence ${ }^{9}$, S. Lamande ${ }^{3}$

${ }^{\prime}$ Victorian Clinical Genetics Services, Melbourne, Australia

${ }^{2}$ Clinical Genetics, Meyer Children's Hospital, Florence, Italy

${ }^{3}$ Murdoch Children's Research Institute, Melbourne, Australia

${ }^{4}$ Northern Regional Genetics Services, Auckland, New Zealand

${ }^{5}$ SA Clinical Genetics Services, Adelaide, Australia

${ }^{6}$ Midcentral District Health Board, Palmerston North, New Zealand

' Genetic Medicine, St Mary's Hospital, Manchester, United Kingdom

${ }^{8}$ Genetics Service, San Raffaele Hospital, Milan, Italy

${ }^{9}$ Genetic Medicine, Sydney Univeristy, NSW, Australia

The transient receptor potential vanilloid-4 protein (TRPV4) is a calcium-permeable ion channel that responds to many different stimuli, is widely expressed, and participates in an extraordinarily wide range of physiologic processes. Autosomal dominant brachyolmia, spondylometaphyseal dysplasia, Kozlowski type (SMDK) and metatropic dysplasia are three distinct skeletal dysplasias which share some common clinical features, including short stature, platyspondyly, and progressive scoliosis. SMDK and metatropic dysplasia also have significant but variable metaphyseal involvement. In the past two years, mutations in the gene encoding TRPV4 have been found to be responsible for these three skeletal phenotypes, confirming that they are allelic disorders, and suggesting that they might represent different parts of a phenotypic spectrum. We clinically, radiographically, and molecularly analyzed 22 patients from 19 different families, all of whom had a clinical diagnosis of one of the three conditions described above: 12 with metatropic dysplasia, 4 with SMKD and 3 with brachyolmia. We identified 8 different muta- 
tions, three previously described and 5 novel, in 18 patients. These data have uncovered new genotype-phenotype correlations in these conditions and suggest that these three conditions represent a continuum of severity with areas of overlap between conditions, even within the same family. These data confirm that metatropic dysplasia, including "lethal" forms, are caused by heterozygous TRPV4 mutations, and strongly suggest that "brachyolmia" represents the mildest expression of SMDK. We hope that these data will add to the understanding of the molecular basis of the emerging TRPV4-related disorders, and identify possible pharmacologic targets for therapy.

\section{THE EXPERIENCE OF PARENTS WHO CONTINUE A PREGNANCY AFTER A DIAGNOSIS OF A LIFE-LIMITING CONDITION IN THEIR BABY}

S. Saya', J. Hynson ${ }^{2}$, A. Horwood ${ }^{3}$, S. Buscombe 4 , J. Hodgson ${ }^{1,5}$

${ }^{\prime}$ Paediatrics, University of Melbourne, Australia

${ }^{2}$ Royal Children's Hospital, Melbourne, Australia

${ }^{3}$ Royal Melbourne Hospital, Melbourne, Australia

${ }^{4}$ Peter MacCallum Familial Cancer Centre, Melbourne, Australia

${ }^{5}$ Genetics Education and Health Research, Murdoch Childrens Research Institute, Melbourne, Australia

Following prenatal diagnosis of a life-limiting condition in their baby, parents may choose to continue their pregnancy aware that their baby will die before or shortly after birth. Perinatal Hospice Care is a structured program of care that is provided in some countries. This program cares for families in this situation by utilizing a multidisciplinary team and providing the opportunity for families to spend the time that they have with their baby in whatever way they wish. Worldwide, little is known about families' experiences after continuing a pregnancy with such a diagnosis and there is no published Australasian data. This exploratory project examines Victorian families' experiences of their baby's life-limiting diagnosis, birth and death. It aims to capture their views on the care and support received at that time, services they found useful and the perceived utility of a perinatal hospice program. Participants were recruited from hospital records and support groups in Victoria, Australia. In-depth, qualitative interviews were conducted with ten parents. Thematic analysis was utilized to identify common themes. Preliminary data analysis reveals that the uncertainty associated with many diagnoses and the responses from health professionals, family and friends has a large impact on families' experiences at this time. Showing respect for the life of the baby is a key theme. All participants were supportive of all aspects of the Perinatal Hospice model. Participants showed great willingness to participate in sensitive research about their experiences. The final results of this study will be presented and the implications for future care provision will be discussed.

\section{EVALUATION OF A WRITTEN CONSENT PROCESS FOR NEWBORN SCREENING IN VICTORIA}

\section{K. L. Scarff',T. Charles ${ }^{2}$, C. Fry ${ }^{1,3}$, L. Gillam ${ }^{3}$ \\ ${ }^{1}$ Murdoch Children's Research Institute, University of Melbourne, Australia \\ ${ }^{2}$ Screening and Cancer Prevention, Victorian Department of Health, Melbourne, Australia \\ ${ }^{3}$ Children's Bioethics Centre, Royal Children's Hospital, Melbourne, Australia}

Newborn screening (NBS) is a vital public health initiative which aims to identify newborn babies at risk of developing certain congenital disorders, so that appropriate treatment can be initiated to reduce morbidity and mortality. After the test, the bloodspot cards are retained for quality assurance, and de-identified samples may be used for health research. Currently in Victoria, parents give verbal consent for their baby to undergo NBS. This presentation will report on an evaluation of a pilot of a written consent process for NBS in Victoria. Previous studies in Australia and overseas have shown that parental awareness and knowledge of NBS is limited, and most are unaware of potential research uses of the stored bloodspots. In order to improve parental understanding of the NBS process, the Victorian Department of Health (DH) has developed a new information brochure for parents outlining the NBS test, and the storage and potential future uses of the bloodspot samples. This brochure includes a form for parents to sign, in order to provide written consent for the screening test and, separately, future use of the bloodspot sample. DH used this brochure in a pilot written consent program initiated at Bendigo Health in February 2010. The parents' perspective on the new written consent process was evaluated by means of a questionnaire, and the refusal rate was monitored. The evaluation showed that introduction of written consent for newborn screening did not result in an increase in test refusals, and that over $90 \%$ of parents consented for their baby's bloodspot card to be used in health research. Parents were not concerned about providing written consent as opposed to verbal consent. The data collected also gives indications of parental understanding of newborn screening, and active decision making concerning the newborn screening test and the use of their child's bloodspot card.

\section{EVALUATION OF A WRITTEN CONSENT PROCESS} FOR NEWBORN SCREENING IN VICTORIA

\section{K. L. Scarff', T. Charles ${ }^{2}$, C. Fry 1,3, L. Gillam ${ }^{3}$}

${ }^{\prime}$ Murdoch Children's Research Institute, University of Melbourne, Australia ${ }^{2}$ Screening \& Cancer Prevention, Victorian Department of Health, Melbourne, Australia ${ }^{3}$ Children's Bioethics Centre, Royal Children's Hospital, Melbourne, Australia

Newborn screening (NBS) is a vital public health initiative which aims to identify newborn babies at risk of developing certain congenital disorders, so that appropriate treatment can be initiated to reduce morbidity and mortality. After the test, the bloodspot cards are retained for quality assurance, and de-identified samples may be used for health research. Currently in Victoria, parents give verbal consent for their baby to undergo NBS. This presentation will report on an evaluation of a pilot of a written consent process for NBS in Victoria. Previous studies in Australia and overseas have shown that parental awareness and knowledge of NBS is limited, and most are unaware of potential research use of the stored bloodspots. In order to improve parental understanding of the NBS process, the Victorian Department of Health (DH) has developed a new information brochure for parents outlining the NBS test, and the storage and potential future uses of the bloodspot samples. This brochure includes a form for parents to sign, in order to provide written consent for the screening test and, separately, future use of the bloodspot sample. DH used this brochure in a pilot written consent program initiated at Bendigo Health in February 2010. The parents' perspective on the new written consent process was evaluated by means of a questionnaire, and the refusal rate was monitored. The evaluation showed that introduction of written consent for newborn screening did not result in an increase in test refusals, and that over $90 \%$ of parents consented for their baby's bloodspot card to be used in health research. Parents were not concerned about providing written consent as opposed to verbal consent. The data collected also gives indications of parental understanding of newborn screening, and active decision making concerning the newborn screening test and the use of their child's bloodspot card.

\section{TESTICULAR FORMATION IN A 46, XY ISOLATED GONADAL DYSGENESIS WITH DAX1 DUPLICATION}

B.A. Setyawati', A. Z. Juniarto', I. P. Miranti' ${ }^{2}$, S. L.S. Drop ${ }^{3}$, S.White ${ }^{4}$, S. M.H. Faradz', J.W. Oosterhuis ${ }^{5}$, L. H.J. Looijenga ${ }^{5}$

${ }^{1}$ Division of Human Genetics, Center for Biomedical Research Faculty of Medicine Diponegoro University (FMDU), Semarang, Indonesia

${ }^{2}$ Department of Anatomical Pathology, Faculty of Medicine Diponegoro University (FMDU), Semarang, Indonesia

${ }^{3}$ Department of Paediatrics, Division of Endocrinology, Erasmus Medical Centre, Rotterdam, Netherlands

${ }^{4}$ Dept of Pediatric, Murdoch Children's Research Institute, University of Melbourne, Melbourne, Australia

${ }_{5}^{5}$ Department of Pathology, Erasmus Medical Centre, Josephine Nefkens Institute, Rotterdam, Netherlands

DAXI (NR0B1), a transcriptional repressor factor, of which the gene is located at X p21.2, plays a critical role in testicular development. As down regulator of the Anti Müllerian Hormone (AMH) it is traditionally considered as an anti-testis factor. Increasing gene 
dosage causes complete sex reversal phenotype in $46, \mathrm{XY}$ individuals as part of more complex anomalies including dysmorphic features and mental retardation. Whilst in contrast deletions and mutations cause Adrenal Hypoplasia Congenital (AHC) and Hypogonadotropic HypoGonadism (HHG). An Indonesian family with three affected girls is reported, one already passed away and the remaining two were 46, XY suggesting X-linked inheritance. Analysis of the available sample of an 18 years old girl with an androgen action disorder manifesting primary amenorrhea, no breasts development and ambiguous genitalia showed no dysmorphic features or mental retardation. Luteinizing Hormone (LH) and Follicle Stimulating Hormone (FSH) were increased with low level of testosterone and minimal response to human ChorioGonadotrophin (hCG) stimulation. No Androgen Receptor (AR) gene mutation was identified. Neither uterus nor ovaries were found laparoscopically, while a remnant of a utricle duct was found. The patient kept her female gender. Gonadectomy was performed for both inguinal testicles and histopathological examination showed Leydig cell hyperplasia lacking of germ cells. Using Multiple Ligation Probe Analysis (MLPA), a submicroscopic duplication at Xp21.2 containing DAXI was identified. Two previous studies of DAXI duplication have been published as cause of isolated gonadal dysgenesis. This is the third case of same duplication in a 46,XY individual with testicular formation, thus demonstrated the importance of $D A X I$ examinations in all cases with isolated $46, \mathrm{XY}$ gonadal dysgenesis.

\section{VARIABILITY IN CYSTIC FIBROSIS SWEAT TEST ANALYSIS REPORTING

\author{
C. Shalhoub', R. Junek ${ }^{4}$, K. Barlow-Stewart ${ }^{3}$, A. Turner', A. Jaffe ${ }^{2}$ \\ ${ }^{I}$ Medical Genetics, Sydney Children's Hospital, Sydney, Australia \\ ${ }^{2}$ Respiratory Department, Sydney Children's Hospital, Sydney, Australia \\ ${ }^{3}$ The Centre for Genetics Education, Sydney, Australia \\ ${ }^{4}$ NSW Newborn Screening Programme, The Children's Hospital Westmead, Sydney, \\ Australia
}

Cystic fibrosis (CF) is the most common (1 in 2500 live-births) life threatening autosomal recessive condition in people of Northern European ancestry, with the classical form characterized by chronic lung disease, pancreatic insufficiency and elevated sweat electrolyte levels. Over 1500 mutations in the cystic fibrosis transmembrane conductance regulator (CFTR) gene are associated with $\mathrm{CF}$ and other CFTR related disorders. NSW newborn screening (NBS) identifies newborns at risk of CF. Babies with a positive NBS test, indicated by a persistent elevated level of immunoreactive trypsinogen and heterozygous status of the most common CFTR gene mutation F508del, are referred for diagnostic sweat chloride analysis, to confirm the diagnosis of CF. It is widely accepted that a sweat chloride level of $>60 \mathrm{mM}$ is diagnostic for classic CF. Australian and UK guidelines also recommend an intermediate range of 40-60 mM. While not all laboratories follow these, an increasing number of individuals are diagnosed with milder and atypical forms of $\mathrm{CF}$ with sweat chloride levels in the intermediate range. Further, some infants are difficult to classify due to having limited or no clinical features and inconclusive diagnostic test results. This makes interpretation of intermediate results problematic and genetic counseling difficult. There is variability in the normal reference ranges utilized by the two laboratories that perform sweat chloride analysis in Sydney, the classifications being $<30 \mathrm{mM}$ and $<40 \mathrm{mM}$. An audit of infants with sweat chloride levels between 30 and $40 \mathrm{mM}$ was undertaken to determine if there are any affected individuals in this range. This audit revealed a number of infants with a diagnosis of $\mathrm{CF}$ that were missed where the normal reference range of $<40 \mathrm{mM}$ was utilized. This reference range variability has already raised genetic counseling issues but also raises the potential for non-treatment of affected individuals in the intermediate range.

\section{COGNITIVE DEVELOPMENT IN CHILDREN WITH EARLY AND CONTINUOUSLY TREATED PHENYLKETONURIA: RESULTS OF AN 8 YEAR LONGITUDINAL STUDY}

R. Sharman ${ }^{1,2}$, K.A. Sullivan ${ }^{1,2}$, R.Young ${ }^{2}$,T. Jones ${ }^{4}$, J. J. McGill ${ }^{3}$

${ }^{\prime}$ School of Psychology and Counseling, Queensland University of Technology, Australia ${ }^{2}$ Institute of Health and Biomedical Innovation, Queensland University of Technology, Australia

${ }^{3}$ Metabolic Medicine, Royal Children's Hospital, Brisbane, Australia

${ }^{4}$ Great Southern Psychiatric and Psychological Services, Albany, Australia

Although children with early and continuously treated PKU now develop normal I.Q., deficits in executive function (EF) are common, leading to a rate of Attention Deficit Disorder up to five times the norm. EF worsens with exposure to higher phenylalanine (phe) levels, although recent research has demonstrated that a high phe:tyrosine ratio (hypothesized to lead to poor dopamine function) has a more negative impact on EF than phe levels alone. 13 children with early and continuously treated PKU were tested at mean age 5.9 years and again at mean age 13.95 years on several neuropsychological measures. 4 children with hyperphe were also tested at both time points and provide a comparison group in analyses. Associations of EF performance with biochemical markers were analyzed. Minor EF impairments were evident in the PKU group by age 6 years and persisted (although did not worsen) into adolescence. Long-term exposure to high phe:tyr ratio and/or low tyrosine independent of phe were significantly associated with EF impairments at both time points. Children with PKU showed a statistically significant decline in a language comprehension task from 6 years to adolescence (going from normal to subnormal), this deficit was associated with lifetime levels of phe. Children with hyperphe demonstrated normal function at both time points, across all measures. Overall, EF impairments were evident by childhood and persisted into adolescence, EF deficits were associated with a high phe:tyr ratio and/or low tyrosine independent of phe. A comprehension task showed a different trajectory, with the children losing function between the ages of 6 and 14 years, largely as a result of long-term exposure to phe. This is a timely reminder that normal function in childhood does not guarantee normal function by adolescence, and can be lost due to poor dietary control, possibly later in development than previously thought.

\section{MANAGING CONSENT TO MICROSATELLITE INSTABILITY TESTING IN CLINICAL PRACTICE}

H. Shipman', A. Clarke', S. Sarangi' ${ }^{2}$ R. Chadwick ${ }^{3}$

${ }^{\prime}$ Medical Genetics, Cardiff University, United Kingdom

${ }^{2}$ Health Communication Research Centre, Cardiff University, United Kingdom

${ }^{3}$ ESRC Centre for Economic and Social Aspects of Genomics, Cardiff University, United Kingdom

Consent takes a central position in biomedical ethics. As technology advances consent must be negotiated in novel contexts where the potential scope and implications remain unknown. Tissue testing may now yield results significant to individuals in terms of diagnosis, treatment decisions and recurrence risk, and also disease risk for their families. This raises new and complex issues of consent and warrants an investigation of the perspectives of the actors. This research considers how consent is understood and approached in clinical tumour testing for features of Lynch Syndrome in the UK. This context introduces further dimensions to consent as the individual seen is clinic is often not eligible to give consent to tumour testing but rather acts as a mediator in the consent process between the clinician and an affected relative. Here we present preliminary findings of how interactional strategies are used by the actors in clinic to negotiate consent in this context during 'consent encounters'. In this discursive thematic analysis we specifically focus on (a) how the novel technology is introduced and responded to and (b) how the co-construction of consent is managed. We demonstrate how discursive strategies of mitigation, repetition and recycling, minimizing burden and contra-indication are used by the actors when negotiating consent. These results, although preliminary, challenge some of the dominant discourses around consent procedures. 
CONSTITUTIONAL MOSAICISM FOR MONOSOMY X AND TRISOMY 18: CLINICAL PRESENTATION AND EVIDENCE FOR THE ORIGIN OF THE ANEUPLOID CELL LINES

T. Shipton', E. Blake', A. Nandini', D. Young' ${ }^{2}$, H. Heussler ${ }^{3}$

${ }^{1}$ Cytogenetics, Pathology Queensland, RBWH, Brisbane, Australia

${ }^{2}$ Molecular Genetics Department, Pathology Queensland, RBWH, Brisbane, Australia

${ }^{3}$ Mater Health Services, Mater Children's Hospital, Brisbane, Australia

Constitutional mosaicism for two distinct chromosome aneuploidies is a rare cytogenetic finding. Only five cases with mosaic aneuploidy of monosomy $\mathrm{X}$ and trisomy 18 are reported. We report a 7-year-old girl with an apparent complete trisomy 18 karyotype in all 15 cells initially analyzed from a peripheral blood lymphocyte culture. However, further FISH investigations performed using the CEP18/X/Y probe (Aneuvysion ${ }^{\circledR}$ kit, Vysis) on interphase nuclei revealed mosaicism with three cell lines: (DXZ1x1,D18Z1x2)[26\%]/(DXZ1x2,D18Z1x3) [48\%]/(DXZ1,D18Z1)x2[26\%]. Therefore, the FISH result contrasted with the non-mosaic cytogenetic analysis and showed that she was mosaic for trisomy 18 and had another cell line with monosomy X. This was an unexpected finding in regard to the patient's clinical presentation. She presented with apparent normal intelligence, severe iron deficiency anaemia, congenital heart defects and short stature in the context of her family. There was a striking absence of any features characteristic of trisomy 18 and the presence of some features of monosomy X. Further investigations to determine the parental origin of the aneuploidies were conducted using PCR analysis of microsatellite markers. This confirmed the supernumerary 18 in the trisomy 18 cell line to be of paternal origin and the single $\mathrm{X}$ chromosome in the monosomy X cell line to be of maternal origin. Further cytogenetic investigations are to be performed on skin biopsies from the child to further characterise the extent of chromosomal mosaicism for these cell lines and the possible mechanisms of formation will be discussed.

\section{MAKING CHOICES: DECIDING TO TEST FOR FETAL} ABNORMALITIES IN PREGNANCY

S. Simpson

Sydney Ultrasound for Women, Sydney, Australia

Sydney Ultrasound for Women has screened over 116,000 women using the Nuchal Translucency and First trimester biochemistry program NT-plus. We have a 91.4\% detection rate for Trisomy 21 and a $90 \%$ detection rate for other aneuploidy. From this population, $30 \%$ of women were over the age of 35 years. It is our practice to discuss diagnostic testing with all women who are flagged as increased risk on the NT-plus assessment $(>1: 300)$ or who are considered to be advanced maternal age (35yrs+). As the decision to proceed to diagnostic testing is not always straightforward, at their consultation we provide information and explore with the couple relevant personal issues to aid their decision-making. However, we find a lot of couples need more time to work through their options before deciding what is the most appropriate path for them. An Australian study found that prenatal genetic counseling sessions were predominantly based on information-giving, with a definite lack of discussion on sensitive topics such as disability and abortion (Hogson et al, 2009). Our team developed a decision aid called Making Choices for women considering prenatal diagnosis. It provides our patients with a tool to take home that covers the information provided in the counseling session, as well as prompting consideration of the more personal issues involved with prenatal diagnosis. Although there have been decision aids published for this exact purpose, we aimed to develop a concise document which targets the specific groups we find struggle with this decision. Our first draft was implemented June 2010, with a questionnaire attached. Permission was obtained from the patients for a follow up phone call. We will present our findings on a data set of $n=100$, to identify if this decision aid is of benefit addition to our genetic counseling with future patients.
THE UTILITY OF CYTOGENETIC SNP ARRAYS FOR THE DETECTION OF CLONAL CHANGES IN MYELOPROLIFERATIVE DISEASE

N. R. Singh ${ }^{1,2}$, W. Stevenson ${ }^{1,2}$, C.Ward ${ }^{2}$

${ }^{I}$ Genetics, Pathology North-RNSH, Sydney, NSW, Australia

${ }^{2}$ Northern Blood Research Center, University of Sydney, Sydney, NSW, Australia

Introduction: The utility of micro array technology has to date provided unprecedented resolution and extended information for copy number changes in constitutional diseases. Limited data is available on the utility of this technology in clonal disease. Materials and Methods: DNA extracted from peripheral blood granulocytes on 11 cases with a diagnosis of myelofibrosis were investigated using the 2.7M Affymetrix Cytogenetic array. Standard Cytogenetic cultures were also established on each patient. Array data was analyzed using the Affymetrix ChAS software. Copy number changes were filtered down to $20 \mathrm{~Kb}$ for losses and $100 \mathrm{~Kb}$ for gains. The UPD Threshold was set above $3 \mathrm{MB}$ at $85 \%$ confidence. Results: 9 of 11 karyotypes correlated with the copy number changes (CNC) detected by the 2.7M Cytogenetic array. Two abnormal karyotypes were not detected by array. Additional CNCs were detected on the array in regions of known significance in hematological disease. In addition, mosaicism shown on software analysis that was associated with copy number changes was helpful in providing further evidence of a somatic rather than a germ line change in some instances. Chromosome $9 \mathrm{p}$ and $1 \mathrm{p}$ showed copy neutral changes with long stretches of homozygosity (LCSH) in 6 and 5 patients respectively. Conclusion: SNP Cytogenetic arrays provide an increased resolution of detection and can be helpful in identifying clonal changes and copy neutral loss of heterozygosity.

\section{CHROMOSOME MOSAICISM DETECTED BY MOLECULAR KARYOTYPING WITH SNP MICROARRAYS}

D. L. Bruno ${ }^{1,2}$, S. M.White ${ }^{2,3,4}$, H. R. Slater ${ }^{1,2,3}$

${ }^{\text {I} V i c t o r i a n ~ C l i n i c a l ~ G e n e t i c s ~ S e r v i c e s, ~ R o y a l ~ C h i l d r e n ' s ~ H o s p i t a l, ~ M e l b o u r n e, ~ A u s t r a l i a ~}$ ${ }^{2}$ Murdoch Childrens Research Institute, Royal Children's Hospital, Melbourne, Australia ${ }^{3}$ Department of Paediatrics, University of Melbourne, Melbourne, Australia ${ }^{4}$ Genetic Health Services Victoria, Royal Children's Hospital, Melbourne, Australia

Genome-wide microarray analysis has an established diagnostic role in detecting pathogenic copy number changes in patients with intellectual disability. This study investigates the utility of the Illumina HumanCytoSNP-12 array for detection of chromosomal mosaicism, and analyses the clinical characteristics of individuals in whom mosaicism was diagnosed. Chromosomal mosaicism was detected in 19 individuals referred for investigation of intellectual disability, developmental delay, abnormal growth, autism or congenital abnormalities. A wide variety of mosaic chromosomal abnormalities were indicated including whole-chromosome trisomies (3 cases), interstitial deletions and duplications (9 cases), terminal deletions and duplications ( 4 cases), complex rearrangements ( 2 cases) and isodisomy ( 2 cases). Notably, seven of the individuals with a previous G-band karyotype analysis on stimulated peripheral blood lymphocytes showed no abnormality. Follow-up investigations on 15 of the cases confirmed the mosaicism and delineated the chromosome abnormalities in detail. A 'spiking' experiment using trisomy-22 and euploid cell lines from a single individual was performed to empirically determine the sensitivity of SNP microarray for detection of chromosomal mosaicism. A minimum sensitivity of $10 \%$ was demonstrated using this approach, an improvement over that provided by array CGH. Analysis of the clinical findings in this patient group showed that two patients had significant limb asymmetry, but otherwise no individuals showed what are regarded as typical clinical manifestations of mosaicism, such as pigmentary disturbance, asymmetry or contractures. No individuals were clinically suspected of mosaicism prior to the array result. None had a clinical diagnosis of hypomelanosis of Ito. In this cohort of 3230 individuals, mosaicism for chromosome abnormalities accounted for $0.6 \%$ of cases. This study shows that the typical phenotype of hypomelanosis of Ito is not a reliable indicator of chromosome mosaicism, and that 
molecular karyotyping with SNP-microarrays is superior to array CGH in the detection of unsuspected mosaicism in children with intellectual disability/ developmental delay.

\section{SECONDARY ACUTE MYELOID LEUKEMIA WITH A NOVEL REARRANGEMENT OF CHROMOSOME 110, ACCOMPANIED BY LOSS OF HETEROZYGOSITY, IN A PATIENT WITH PRECURSOR B CELL ALL AT DIAGNOSIS}

S. Diaz', D. R. Catchpoole², T. Kilo , D. Guo ${ }^{2}$, S. Kellie ${ }^{4}$, E. Smith'

${ }^{\prime}$ Cytogenetics, Children's Hospital at Westmead, Sydney, Australia

${ }^{2}$ Children's Hospital at Westmead, Tumour Bank, Oncology Research Unit, Sydney, Australia

${ }^{3}$ Haematology, Children's Hospital at Westmead, Sydney, Australia

${ }^{4}$ Oncology, Children's Hospital at Westmead, Sydney, Australia

Abnormalities of the mixed lineage leukemia (MLL) gene, located at $11 \mathrm{q} 23$, are ubiquitous, seen in both adult and paediatric ALL, de novo and secondary AML and as a therapy related abnormality. We describe a 14-year-old girl with precursor B ALL, managed by standard protocols of treatment. The diagnostic bone marrow cytogenetics was normal, there was no fusion seen on FISH for ETV 6/RUNX1, although there was deletion of one ETV 6 signal, and there was no fusion by molecular studies for $t(12 ; 21), \mathrm{t}(4 ; 11)$, $\mathrm{t}(9 ; 22), \mathrm{t}(1 ; 19)$. Initially, there was a good response, but at the end of maintenance therapy, she unexpectedly showed signs of myelodysplasia and developed acute myeloid leukemia. At this time, bone marrow cytogenetics showed an acquired paracentric inversion within 11q, which was present in two copies without a normal chromosome 11 [inv(11)(q21q23)x2]. Loss of heterozygosity (LOH) for the MLL gene on 11q was confirmed by FISH, and genome wide SNP studies identified LOH for nearly the whole of the 11q, a region of $\sim 400 \mathrm{Mb}$. This unique rearrangement, present $\sim 2$ months before overt leukemia, responded well to therapy prior to bone marrow transplantation. The questions posed by the case relate to the significance of deletion of ETV 6 without fusion at diagnosis of ALL (barely touched in the literature) and to what extent the (novel) $\mathrm{LOH}$ of MLL was therapy related or harbinger of a new acute leukemia.

\section{NOVEL KRAS MUTATION IN CARDIO-FACIO-CUTANEOUS SYNDROME: TRANSMISSION THROUGH TWO GENERATIONS OF A FAMILY}

\section{Z. Stark', M. Zenker ${ }^{3}$, R. Savarirayan ${ }^{1,2}$ \\ ${ }^{\prime}$ Clinical Genetics, Genetic Health Services Victoria, Melbourne, Australia \\ ${ }^{2}$ Department of Paediatrics, University of Melbourne, Australia \\ ${ }^{3}$ Institute of Human Genetics, Otto-von-Guericke University, Germany}

Cardio-facio-cutaneous (CFC) syndrome is characterized by typical facial features, cardiac defects, ectodermal anomalies, and intellectual disability. CFC is a Ras-MAPK pathway disorder and clinical features overlap other related conditions including Noonan and Costello syndromes. KRAS mutations account for $<5 \%$ of reported CFC cases, the remainder caused by mutations in BRAF, MEKI and $M E K 2$. We describe a mother and son with a clinical diagnosis of CFC syndrome, based on the typical phenotype. In addition, the mother had significant peripheral neuropathy complicated by Charcot arthropathy. Sequence analysis of the KRAS gene revealed a novel missense mutation c.211T C (p.Y71H), which results in substitution of a highly conserved tyrosine residue by histidine. CFC is usually considered a sporadic disorder with only one previous report of a vertically transmitted mutation in MEK2. The Ras/MAPK pathway is critical for both male and female reproductive function, but it is unclear whether the paucity of vertical transmission in CFC is due to direct or indirect impacts on reproductive fitness. This case report further expands our understanding of KRAS germline mutations to include peripheral neuropathy and has important implications for reproductive risk counseling in this condition.
REVIEW OF THE USE OF BOADICEA IN DETERMINING ELIGIBILITY FOR BRCA1 AND BRCA2 MUTATION SCREENING UNDER THE PUBLIC HEALTH SYSTEM

S.J. Steele ${ }^{1,2}$, L.Warwick ${ }^{3}$, K. Tucker ${ }^{4}$

${ }^{1}$ Canberra Hospital, ACT Genetics Service, Canberra, Australia

${ }^{3}$ Canberra Hospital, ACT Genetics Service, Canberra, Australia

${ }^{4}$ Prince of Wales Hospital, Hereditary Cancer Clinic, Sydney, Australia

Background: The computer algorithm BOADICEA is used to calculate the carrier probabilities of BRCA1 and BRCA2 mutations in patients with a personal or family history of breast and/or ovarian cancer. The use of BOADICEA allows those patients at high risk of carrying a mutation to be identified and offered mutation screening through public health system funding. Previously, the eligibility criteria for ACT patients to be offered mutation screening was based on NBOCC criteria and clinical judgement. In the 12 month period between July 2009 and June 2010, the use of BOADICEA was integrated into this criteria. Eligible patients have a $10 \%$ or greater risk of carrying a BRCA1 or BRCA2 mutation as predicted by BOADICEA. Purpose: This report compares BOADICEA predictions with the mutation status of patients ascertained by MLPA and DNA sequencing to assess the beneficence and cost-effectiveness of the revised eligibility criteria. Method: Patients attending the ACT Genetics Service between July 2009 and June 2010 for discussion of personal or family history of breast and/or ovarian cancer were reviewed. Family history, BOADICEA predictions, undertaking of genetic testing and mutation status were noted. Results: Of the 118 patients attending the ACT Genetics Service, 36 patients underwent BRCA1 and BRCA2 mutation screening. Fifteen patients were above the $10 \%$ threshold, in which two mutations and two variants of unknown clinical significance were identified. Of the 21 patients offered mutation screening below the $10 \%$ threshold, three variants and one mutation were identified. The latter mutation was identified in a patient carrying a $6.81 \%$ mutation risk. Conclusion: This report reviews the beneficence and cost-effectiveness of the use of BOADICEA in determining eligibility for BRCA1 and BRCA2 mutation screening, in which screening is restricted to patients above the $10 \%$ threshold. The importance of clinical judgement in offering mutation screening is emphasized, as it takes into account additional risk factors not considered by computer algorithms.

\section{OUTREACH RURAL GENETIC COUNSELING - DEVELOPING A NEW MODEL OF CARE \\ J. Stofmeel', G. Turner ${ }^{2}$, K. Frumar ${ }^{3}$, B. Hopper ${ }^{4}$, C. Sorensen ${ }^{3}$ \\ ${ }^{\prime}$ Coffs/Clarence Network, North Coast Area Health Service, Coffs Harbour, Australia \\ ${ }^{2}$ Mid North Coast Genetic Service, NSW, Australia \\ ${ }^{3}$ Hastings/Macleay Network, North Coast Area Health Service, Port Macquarie, Australia \\ ${ }^{4}$ Manning Cluster, Hunter New England Area Health Service, Taree, Australia}

Outreach clinical genetic services are provided in rural centres in most states throughout Australasia. These genetic counsellors work alongside visiting specialists from the major genetic centres. The role of rural outreach genetic counsellors has evolved since they were first established in the late 1980s, with multiple challenges and opportunities faced. Resource limitations and increased demand means that new ways to give rural clients and families' timely and appropriate advice and counseling are constantly being sought. Information was received through 52 questionnaires from genetic counsellors throughout Australasia and the review of work practice in three outreach genetic settings on the mid-north coast NSW. This revealed that $70 \%$ of outreach genetic counsellors were board certified, $70 \%$ had over 7 years of experience, compared with $50 \%$ and $58 \%$ respectively for genetic counsellors in metro units. Outreach genetic counsellors felt more confident in their ability to work independently than metro-based genetic counsellors and were all ordering some type of genetic test. Given the increasing demand for genetic services and the absence of an on-site clinical geneticist, the ability to order certain genetic tests increases efficiency and client satisfaction. A review of all general clients seen at three outreach 
clinics over a two-year period by the visiting clinical geneticist and the genetic counsellors, showed that almost half of clients referred to the service could be seen independently by the genetic counsellor or with only minimal advice by phone from the clinical geneticist. The continued professional growth of genetic counsellors coupled with present economic restraints is changing models of access and service delivery in creative ways. Outreach genetic counsellors function with a degree of isolation not encountered in the metropolitan units and there is a need to have access to ongoing education and a good working relationship with their peers.

DIETARY MANAGEMENT OF AN INFANT WITH 2-METHYL3-HYDROXYBUTYRYL-COA DEHYDROGENASE (MHBD) DEFICIENCY IN SOUTH AUSTRALIA

A. L. Sweeney', D. Bratkovic' ${ }^{2}$, J. R. Harrison ${ }^{2}$, E. Ranieri' ${ }^{2}$

${ }^{\prime}$ Nutrition Department, CYWHS, Adelaide, Australia

${ }^{2} S A$ Pathology, WCH, Adelaide, Australia

Aim: To describe the dietary management of a female infant diagnosed on newborn screening with MHBD. The acylcarnitine profile showed the elevation of the acylcarnitines; $\mathrm{C} 4 \mathrm{OH}, \mathrm{C} 5: 1 \& \mathrm{C} 5 \mathrm{OH}$ and the related ratios. MHBD is an x-linked recessive disorder of isoleucine (isoleu) and alpha-methyl-branched-chain-fatty acid metabolism resulting in increased urinary excretion of two metabolites-tiglylglycine (C5:1) and 2-methyl-3-hydroxybutyric acid $(\mathrm{C} 4 \mathrm{OH})$. MHBD is associated with poor neurological development and it has been reported in older children a diet low in isoleu has resulted in a decrease of the raised urinary metabolites and stabilization of neurological symptoms. Case report: Breastfed infant diagnosed on day 3 newborn screening with elevated acylcarnitines. Diagnosis of MHBD was confirmed on urine organic acids at 2 weeks of age which showed raised tiglylglycine and 2-methyl-3hydroxybutyric acid, $83(\mathrm{RR}<1)$ and 242 units $(\mathrm{RR}<20)$ respectively. Method: A low isoleu diet was commenced at 24 days of age by substituting every second breastfeed with a MSUD infant formula with added valine and leucine. The mother chose to cease breastfeeding 2 weeks later and an infant formula was added to the dietary plan. The aim is to reduce the raised urinary metabolites, while still providing adequate amounts of isoleu, for normal growth and development. Plasma amino acids, urinary metabolites, growth and development are measured monthly and diet adjusted as appropriate. Results: At 4 months of age she was receiving $31 \mathrm{mg}$ isoleu/ $\mathrm{kg} /$ day from her formula. Urinary metabolites tiglylglycine and 2-methyl-3hydroxybutyric acid had decreased to 10 and 52 units respectively. Plasma isoleu at 3 weeks of age was $70 \mu \mathrm{mol} / \mathrm{l}$ (range 25-55) and at 4 months of age $36 \mu \mathrm{mol} / \mathrm{l}$. Conclusion: We have been able to achieve a reduction in urinary metabolites, whilst achieving normal growth and development in a 4-month-old infant. Further information will be available at the conference.

\section{A MODERN APPROACH TO THE SEARCH FOR MODIFYING GENETIC LOCI INFLUENCING THE HIGH BREAST CANCER INCIDENCE SEEN IN AN AUSTRALIAN HNPCC/LYNCH SYNDROME COHORT}

B.A.Talseth-Palmer ${ }^{1,2}$, E. Holliday ${ }^{1,2}$, T. Evans ${ }^{1,2}$, M. McPhillips ${ }^{3}$, M. McEvoy, , J.Attia ${ }^{4}$,

R.J. Scott $1.2,3$

${ }^{\text {I} S c h o o l ~ o f ~ B i o m e d i c a l ~ S c i e n c e s ~ a n d ~ P h a r m a c y, ~ U n i v e r s i t y ~ o f ~ N e w c a s t l e, ~ A u s t r a l i a ~}$ ${ }^{2}$ Huter Medical Research Institute, Newcastle, Australia

${ }^{3}$ Division of Genetics, Hunter Area Pathology Service, Newcastle, Australia ${ }^{4}$ School of Medicine and Public Health, University of Newcastle, Australia

Hereditary non-polyposis colorectal cancer (HNPCC)/Lynch syndrome (LS) is an autosomal dominantly inherited cancer syndrome characterized by early-onset epithelial cancers. Modifier genes may contribute to variation in disease expression, and reports have suggested that breast cancer belongs within the context of HNPCC/Lynch syndrome. The aim of the current study was to identify genomic aberrations including copy number $(\mathrm{CN})$ gains and $\mathrm{CN}$ losses which differentiate: a) breast cancer positive families within the HNPCC group and; b) HNPCC-positive individuals from healthy controls. A total of 100 individuals diagnosed with HNPCC and 384 healthy controls were included in the study. All individuals were genotyped using the Illumina Human610-Quad SNP array. Initially the samples were processed in GenomeStudio (Illumina Inc.) before analyzed utilizing Nexus software, Version 5 (BioDiscovery). SNPRank segmentation algorithms was used in Nexus with a significance threshold of 0.01 optimized by NSR (proportion of unique CNV SNPs found in only 1 sample divided by the average number of CNV SNPs called per sample) and a minimum of 5 probes per segment. We detected a CN gain on chromosome 19q12 that was observed in $40 \%$ of the 10 HNPCC Caner+/Breast Cancer+ cases but in none of the controls ( $p$ value of 1.05E-06; Q-value of 6.33E-04), which is also significant if the 38 families with breast in a family member are compared to controls. Two regions shows $\mathrm{CN}$ gain, chromosome $7 \mathrm{q} 11.21$ and $16 \mathrm{p} 11.2$, that are significantly different between 96 HNPCC probands (40\%) compared to controls (1-4\%). Comparison of total and average CNV length (CNV burden) between groups revealed a greater total genomic CNV length $(p=$ $.0006)$ and average CNV length $(p=.0044)$ in the 96 HNPCC compared to the 384 controls. In conclusion, preliminary CNV analysis identified a locus associated with breast cancer in HNPCC/LS patients and 2 loci differentiating HNPCC/LS patients from healthy controls. The CNV burden and $4 / 5 \mathrm{CN}$ gain results have been confirmed with Quanti-SNP.

YPEL1 IN CRANIOFACIAL DEVELOPMENT AND THE DISTAL 22011.2 SYNDROMES

T.Y.Tan ${ }^{1,2,3}$, A. Collins ${ }^{5}$, P.A. James', G. McGillivray', Z. Stark', C.T. Gordon',

R. J. Leventer ${ }^{3,4}$, K. Pope', R. Forbes', J.A. Crolla', D. Ganesamoorthy',

T. Burgess', D. L. Bruno', H. R. Slater', D. J.Amor ${ }^{1,3}$, P. G. Farlie ${ }^{2,3}$

${ }^{\prime}$ Genetic Health Services Victoria, Murdoch Children's Research Institute, Melbourne, Australia

${ }^{2}$ Craniofacial Development, Murdoch Children's Research Institute, Melbourne,

${ }^{2}$ Craniofacial

${ }^{3}$ Department of Paediatrics, University of Melbourne, Australia

${ }^{4}$ Neurology, Royal Children's Hospital, Melbourne, Australia

${ }^{5}$ Wessex Clinical Genetics Service, Princess Anne Hospital, Southampton,

United Kingdom

${ }^{6}$ Wessex Regional Genetics Laboratory, Salisbury District Hospital, Salisbury, United Kingdom

Microarrays have facilitated the recognition of copy number abnormalities distal to the typically deleted proximal $22 \mathrm{q} 11.2$ region. The distal 22q11.2 phenotypes are variable and clinically distinct to those of the proximal region. We present clinical and molecular characterisation of five families with copy number abnormalities affecting distal 22q11.2. Novel clinical features expand the phenotypic spectrum, including diaphragmatic hernia and Mullerian fusion anomalies associated with the distal microdeletion syndrome, and frontomedial polymicrogyria and callosal agenesis associated with the distal microduplication syndrome. Additionally, the diagnosis of a distal 22q11.2 microdeletion in a patient with Goldenhar syndrome confirms that this region is a potential locus for this causally heterogeneous disorder. Microarray analysis of 12 patients with Goldenhar syndrome and related phenotypes will be presented. We also present functional characterisation of a distal 22q11.2 gene, Ypell, in avian craniofacial development. Analysis of endogenous Ypell supports the notion that it plays a role in the development of structures derived from the pharyngeal arches and that it localises to the nucleus. Retroviral-mediated in vivo overexpression of Ypel1 causes abnormal mandibular and ocular morphogenesis associated with increased apoptosis, with several lines of evidence suggesting involvement of the Bmp/Msx pathway. Detailed characterisation of the Ypell-induced phenotypic abnormalities in chick include analyses of chondrocyte disorganisation, tissue proliferation and cell death and in vitro and in vivo expression of genes involved in craniofacial development. We examine the relationship between Ypell in avian craniofacial development and the dysmorphology observed in the human distal 22q11.2 syndromes, Goldenhar syndrome and related phenotypes. 


\section{THREE ADULT SIBLINGS WITH \\ MUCOPOLYSACCHARIDOSIS TYPE II (HUNTER SYNDROME): A REPORT ON CLINICAL HETEROGENEITY AND 12 MONTHS OF THERAPY WITH IDURSULFASE}

\section{C.Tchan, K.T. Devine, D. O. Sillence}

Department of Genetic Medicine, Westmead Hospital, Sydney, Australia

Mucopolysaccharidosis type II (MPS II - Hunter syndrome) is a rare $\mathrm{X}$-linked recessive disease of lysosomal glycosaminoglycan metabolism leading to a systemic storage disorder. We report on three adult brothers (aged 46 to 52 years) with attenuated Hunter syndrome after 12 months of enzyme replacement therapy with idursulfase. Prior to enzyme replacement therapy each had serious complications of their disease: in addition to all requiring urgent cervical spinal canal decompressions in middle age, one required emergency aortic and mitral valve surgery, another had stage IV airways disease and the third had acute glaucoma resulting in unilateral blindness. One brother discontinued therapy after 12 months. The other two brothers reported subjective improvements in energy and exercise tolerance.

\section{A FAMILIAL PTEN MUTATION CAUSING MACROCEPHALY AND ASPERGER SYNDROME AND THE UNUSUAL FEATURES OF PHEOCHROMOCYTOMA AND POLYDACTYLY}

E.Thompson', N. Spurrier ${ }^{2}$, G. Suthers ${ }^{3}$, L. Rawlings ${ }^{3}$

'SA Clinical Genetics Service, SA Pathology, Women's and Children's Hospital Site, Adelaide, Australia

${ }^{2}$ Paediatrics, Flinders Medical Centre, Adelaide, Australia

${ }^{3}$ Familial Cancer Unit, SA Clinical Genetics Service, SA Pathology, Women's and Children's Hospital Site, Adelaide, Australia

${ }^{4}$ SA Pathology, IMVS, Familial Cancer Laboratory, Adelaide, Australia

The proband, a female aged 9 years, was referred at the age of $4 \frac{1}{2}$ years because of dysmorphic features and macrocephaly. Her intellectual development was advanced but motor development was delayed. She had previously had a hamartoma removed from her right eyebrow. All growth parameters were over the 97th centile. Her head circumference was $6 \mathrm{SD}$ above the mean. She had a nubbin of tissue on the lateral border of the left 5th finger. Her father had a pheochromocytoma at age 41 years and had recently been diagnosed with Hashimoto's thyroiditis. He was of normal intelligence and was overweight. His head circumference was $64 \mathrm{cms}$ ( $8 \mathrm{SD}$ above the mean). Routine lymphocyte chromosomes, fragile X DNA test and MRI brain scan were normal. Recently, the family was reviewed. The proband had been diagnosed with Asperger syndrome and had an intention tremor. Her MRI brain scan was again normal. The father had been diagnosed with a renal cancer. A chromosome microarray was normal. A heterozygous missense variant was identified in the proband in exon 6 of the PTEN gene (c.158G>A, p.Arg173His) which is likely to be pathogenic based on previous studies. Her father is being tested and is assumed to carry the same mutation. Various phenotypes are associated with PTEN mutations including Cowden syndrome, Bannayan-Riley-Ruvalcaba syndrome, Proteus syndrome and Lhermitte-Duclos disease. In addition, a few patients with macrocephaly and autism spectrum disorder have PTEN mutations. Thyroiditis and renal cancer are well known associated abnormalities. Our proband will require surveillance as an adult for breast, colon, renal and thyroid cancers. Pheochromocytoma and polydactyly are unusual in PTEN disorders but have been described.

\section{UPDATING THE ASIEM GALACTOSEMIA HANDBOOK - CHALLENGES AND CHANGES}

\section{S.Thompson, M. Netting, C. Bonifant, R.Akroyd}

Australasian Society for Inborn Errors of Metabolism (ASIEM), Galactosaemia Handbook Working Party, Australia

The Australasian Society for Inborn Errors (ASIEM) Galactosemia Handbook 1998 standardized dietary restrictions for galactosaemia in Australia and New Zealand. Since that time there have been changes to food labeling laws, increased availability of modified foods, public concern about prolonged soy intake by children and ongoing research into management of galactosaemia. Unfortunately best practice dietary management does not necessarily result in a good outcome for the child with galactosaemia, except in the acute toxicity phase, and the mechanisms of poor outcome are not yet understood. Diet management practices differ throughout the world with some clinics restricting as much galactose as possible for life and others (such as the ASIEM) restricting major food sources. The challenge in updating the Galactosemia Handbook in 2010 has been to provide clear practical guidance on dietary management of galactosemia for parents and patients, based on limited Australasian data on galactose content of foods, ongoing use of lactose as a flavour carrier in processed foods and uncertainty on the extent of galactose restriction needed. The project undertaken by a Working Party of Dietitians has reviewed the literature, consulted food industry and experts and circulated an updated handbook draft and background paper to ASIEM members and families of patients with galactosaemia for comment. The handbook will be available as a pdf file in the ASIEM resources section of the HGSA website for printing by members as needed. This will allow updates as best practice changes. The major changes in the 2010 handbook include:

- information on managing cessation of breastfeeding

- new recommendations on suitable infant formula, milk substitutes, cheese and fermented soy products

- updated recommendations on calcium intake and bone health

- background information for parents on normal speech development

- updated information on food label reading and use of food allergen warnings on pack.

\section{A SEVERE NEURODEVELOPMENTAL DISORDER ('CONGENITAL VARIANT OF RETT SYNDROME') IN A BOY WITH A DE NOVO 14012 DELETION DETECTED ON MICROARRAY}

\section{E.Thompson', J. Nicholl ${ }^{2}$,W.Waters ${ }^{2}$, M. Harbord ${ }^{3}$}

SA Clinical Genetics Service, SA Pathology, Women's and Children's Hospital Site, Adelaide, Australia

${ }^{2}$ Genetics and Molecular Pathology, SA Pathology, Women's and Children's Hospital Site, Adelaide, Australia

${ }^{3}$ Neurology, Flinders Medical Centre, Adelaide, Australia

This 6-year-old boy has severe microcephaly, severe intellectual disability, spastic quadriplegia, choreoathetosis and epilepsy. Birth weight and length at term were on the 50th centile and head circumference was on the 10th $(33.5 \mathrm{cms})$. By 4 months of age, head circumference was on the 2 nd centile and he had delayed development. Seizures and choreoathetosis began during the first year. Now, he cannot stand or walk unaided and has no speech development. He is not dysmorphic facially. Brain MRI scans showed a normal body of the corpus callosum but the splenium and genu were underdeveloped. An Agilent Technologies platform, 8plex 60K oligonucleotide array with dye swap was performed which showed an interstitial $14 \mathrm{q} 12$ deletion of $4.12 \mathrm{Mb}$. The deletion was confirmed in metaphase cells of the patient using two BAC probes, RP11-805P02 and RP11-113E19 which both map within the deleted region. Neither parent carried the deletion using the two BAC probes. The deleted region contains at least 3 known genes: NOVA1, FOXG1 and PRKD1. Rett syndrome is a severe neurodevelopmental disorder with normal development for the first 6-18 months, followed by a period of developmental stagnation, then regression and later stability. Loss of purposeful hand movement, seizures and acquired microcephaly are among the typical features. Most cases are female and caused by mutations in the X-linked MECP2 gene. Several variants have been described including the congenital variant with onset in the first few months of life due to deletion of or mutations in the FOXG1 gene. This gene encodes the fork-head box protein G1, a winged-helix transcriptional repressor which is critical for forebrain development. Other patients with FOXG1-associated congenital Rett syndrome have also had early-onset choreoathetosis and a hypoplastic corpus callosum which may be useful pointers to the diagnosis. 
PRE-IMPLANTATION GENETIC DIAGNOSIS FOR THE MITOCHONDRIAL DNA M. 8993T>G MUTATION YIELDS A HEALTHY CHILD AND EVIDENCE OF SELECTION FOR GERM CELLS WITH HIGH MUTANT LOADS

\section{R. Thorburn ${ }^{1,2}$, L.Wilton ${ }^{3}$, S. Stock-Myer ${ }^{3}$, D. C. Samuels ${ }^{4}$}

Mitochondrial Research \& VCGS Pathology, Murdoch Childrens Research Institute Melbourne, Australia

Department of Paediatrics, University of Melbourne, Australia

Melbourne IVF, Melbourne, Australia

Center for Human Genetics Research, Vanderbilt University Medical Center, Nashville, United States

We describe pre-implantation genetic diagnosis (PGD) for prevention of mitochondrial disease caused by the m.8993T $>\mathrm{G}$ mutation. The proband died at 2 years of age with a m.8993T $>\mathrm{G}$ mutant load of $>95 \%$ in various tissues. The healthy mother had a mutant load of $35 \%$ in blood and buccal cells. Prenatal diagnosis in two subsequent pregnancies identified mutant loads $>95 \%$ on both occasions and the pregnancies were terminated. A quantitative fluorescent PCR-RFLP test was used over three cycles of IVF and PGD to measure mutant loads. 13 of 17 embryos had $>95 \%$ mutant load and were predicted to be affected. Three embryos had moderate to low mutant loads (30$40 \%$ ). The final embryo had a low mutant load of $2.5 \%$ and was transferred, resulting in a pregnancy and a healthy baby girl with a mutant load of approximately $4 \%$ in cord blood and placenta. We determined the mutant load in an additional 17 oocytes (16 had mutant loads $>80 \%$ ) as well as individual blastomeres from the affected embryos. Mutant load was consistent between all sister blastomeres tested (49 in all), strongly supporting the concept that the mutant load measurement from a single blastomere is representative of the entire embryo. Given the large numbers of oocytes and embryos with high mutant load, we conclude that PGD can be an extremely useful reproductive option for carriers of mitochondrial DNA mutations. Cumulative probability distribution of the oocyte and embryo data was not compatible with neutral drift theory. Recent data from zebrafish and flies suggest that mitochondria with high membrane potential may be preferentially transmitted to germ cells. The m.8993T $>\mathrm{G}$ mutation is unusual in causing an increase rather than a decrease in mitochondrial membrane potential at high mutant loads. We propose that this may explain why this mutation shows such marked skewing toward high mutant loads.

\section{A CASE STUDY IN PYRUVATE DEHYDROGENASE DEFICIENCY AND THE IMPORTANCE OF CLINICAL INFORMATION FOR DIAGNOSIS}

S.Tregoning',W. Salter', E. Oldaker', A. Boneh', S.Thompson², I.Alexander²,

K. Bhattacharya', R. M. Brown', G. K. Brown', D. R.Thorburn'

${ }^{\prime}$ Mitochondrial Research, Murdoch Childrens Research Institute, Melbourne, Australia

The Children's Hospital at Westmead, Sydney, Australia

Biochemistry Department, University of Oxford, United Kingdom

Pyruvate dehydrogenase complex (PDHC) deficiency is one of the more common biochemical diagnoses caused by abnormal mitochondrial metabolism. PDHC is a mitochondrial matrix enzyme that links glycolysis to the Tricarboxylic acid cycle by converting pyruvate into acetyl CoA. PDHC deficiency causes symptoms that overlap those of other mitochondrial diseases, including fatal infantile lactic acidosis, childhood neurodegenerative diseases such as Leigh syndrome and milder later-onset disorders such as episodic ataxia. PDHC deficiency can have X-linked or recessive inheritance, with 40 of the 59 patients diagnosed by our MCRI laboratory having had causative mutations identified in the chromosome X-encoded PDHAl gene. There are a number of difficulties associated with diagnosing PDHC deficiency. Fibroblast PDHC activity can be normal in patients with bona fide PDHC defects due to random X-inactivation in girls and thiamineresponsive defects in boys. Subtle differences in cell culture conditions and assay protocols between different centres can also lead to inconsistency in results of enzymatic testing within and between centres. Enzyme diagnosis is often a prerequisite for molecular investigations, so it is critical to consider factors such as clinical presentation, plasma and CSF lactate/pyruvate ratios and response to dietary modification in evaluating patients with normal or equivocal PDHC activity. The importance of the clinical information and follow up can be seen with our case study, where PDHC deficiency was initially diagnosed via fibroblast enzyme assay $(40 \%$ and $27 \%$ residual activity relative to citrate synthase, in two subcultures) in a boy with developmental delay, hypotonia, neuropathy, hyperlacticacidemia and dysmorphism. However, testing in the UK and repeat testing at MCRI gave equivocal results of $63 \%$ to $77 \%$ residual activity. Strong clinical suspicion based upon response to ketogenic diet, including hypoketotic, hypotonic episodes during temporary dietary lapses, with prompt recovery upon resumption of diet led to identification of a pathogenic PDHAI mutation.

\section{NEWBORN SCREENING FOR CARNITINE UPTAKE DEFECT IN VICTORIA}

L.Trieu', J.Yaplito-Lee', N.Tzanakos', I. Francis', H. Peters', A. Boneh',

K. Carpenter ${ }^{2}$, N. Longo ${ }^{3}$, J. Pitt ${ }^{1}$

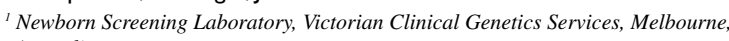
Australia

${ }^{2}$ NSW Biochemical Genetics, Westmead Children's Hospital, Sydney, Australia

${ }^{3}$ University of Utah, United States

Background: Carnitine Uptake deficiency (CUD) is an autosomal recessive disorder which leads to defective plasma membrane carnitine transport in the kidney and muscle. CUD patients may present at birth and later with hypoketotic hypoglycemia, hepatic encephalopathy or progressive cardiomyopathy and muscle weakness. They typically present with low plasma free carnitine levels. Methods: Since the introduction of expanded Newborn screening in April 2002 until June 2010, the Victorian Newborn Screening laboratory has screened approximately 613,000 newborns. In this study, we reviewed babies with low free carnitine $(\mathrm{C} 0)$ detected by newborn screening. Results: 383 babies were detected with $\mathrm{C} 0<0.45 \mathrm{MoM}$ ( $c$ a $8.0 \mu \mathrm{mol} / \mathrm{L}$ ) on the first sample. 26 had low carnitine confirmed on a repeat sample. Of these, 7 newborns had CUD and 5 had presumptive CUD pending further testing. We also identified 3 maternal CUD cases ( 1 confirmed and 2 presumptive) and 1 confirmed CUD paternal case. Overall, low free carnitine had a positive predictive value of at least $1.8 \%$ for CUD with a false positive rate of $\mathrm{ca}$ $0.06 \%$. Our cohort of CUD cases are asymptomatic. They are on carnitine supplementation at $100 \mathrm{mg} / \mathrm{kg} /$ day. They have been reviewed regularly for 42 months (range: 2 months-60 months). Adult untreated CUD cases reported tiredness, low energy and muscle pains. We have also collated data (age at collection, feeds and TPN) relative to $\mathrm{C} 0$ levels at different gestations. Most premature infants have typically normal $\mathrm{C} 0$ levels on the first screen but significantly lower levels on later samples. Conclusion: CUD has an incidence of at least 1:88,000 in the Victorian population. Symptoms appear uncommon in infancy but further studies are required to determine possible long-term consequences. Affected parents are identified through cascade screening and may be symptomatic.

\section{WHAT DO WE SEE AND WHAT DO WE DO IN OUTREACH GENETICS CLINICS}

G.Turner, J. Stofmeel, B. Hopper, K. Frumar, C. Sorensen

Mid North Coast Genetic Service, NSW, Australia

253 families were seen in the monthly and bimonthly clinics in $1 / 1 / 2008-31 / 12 / 2009$ in three settings in the Mid North Coast of NSW( cancer referrals excluded). In 166 the proband was under the age of $18 y$ rs of whom in 122 the presenting problem was autism or developmental disability. In this group 9 had a known cytogenetic abnormality and 20 had a diagnosis revealed by molecular array. The geneticist confirmed or established a probable diagnosis in 38 (30\%) but no diagnosis was achieved in 50\%. Three 3 generational array families will be described demonstrating variability in clinical expression. In $34 \%$ the proband was adult where in $60 \%$ the questions were related to dominant, recessive or $\mathrm{X}$ linked conditions with a known clinical diagnosis or gene mutation wishing for presymptomatic or symptomatic gene testing. The remainder were 
related to translocations, abnormal pregnancies and miscellaneous other conditions. In conclusion we suggest that in the younger age group almost all should have had an array rather than a karyotype ordered by the pediatrician before referral and this is now happening. Also, prior to the clinic visit it is wise, to avoid dissatisfaction, for the genetic counsellor to ensure that parents are aware that a diagnosis in developmental disability is still achieved in only $50 \%$. In most adult referrals we suggest that the genetic counsellor could act independently of the medical geneticist with backup by phone and email.

\section{PREGNANCY FOLLOWING PGD FOR GENERALIZED ARTERIAL CALCIFICATION OF INFANCY}

A. P.Twomey, L. J.Wilton, S. Stock-Myer

Preimplantation Genetics, Melbourne IVF, Melbourne, Australia

Generalized arterial calcification of infancy (GACI) is a rare autosomal recessive disorder involving widespread calcification of arteries and soft tissues. Most affected patients die from myocardial infarction in the first 6 months of life. Mutations which inactivate ecto-nucleotide pyrophosphatase/phosphodiesterase 1 (ENPP1) are the main cause of GACI. A couple receiving fertility treatment through Melbourne IVF conceived a child who was relatively well for the first three months of life. The child sadly died at about four months of age following cardiac failure. DNA diagnosis demonstrated that the couple were both heterozygous for mutations in the ENPP1 gene [c.1412A >G (p.Y471C) \& c.2311+1 G>A] and were thus carriers of GACI. The couple chose to have preimplantation genetic diagnosis (PGD) for subsequent IVF cycles. A novel single cell multiplex PCR test was established to diagnose GACI from individual cells removed from 3-day-old human preimplantation embryos. This was achieved using a combination of allele specific primers to detect the two mutations as well as primers to amplify four STR markers linked to the ENPP1 gene. The couple has had two cycles of IVF with PGD. In the first cycle three embryos were tested and all were found to carry both of the parent's mutations. In the second cycle eight embryos were tested. Four were shown to be carrying both of the mutations (affected embryos), two were diagnosed as carriers of the disease and one embryo did not carry either mutation (unaffected embryo). One result was unclear. An embryo carrying the paternal mutation was transferred to the patient on Day 4 resulting in an ongoing singleton pregnancy. This result has been confirmed by prenatal diagnosis. To our knowledge, this is the first worldwide report of PGD for GACI.

\section{HYPERAMMONEMIA VS HYPOGLYCEMIA \\ A TALE OF TWO UREA CYCLE DEFECTS}

\section{E.Vandervliet}

Dept Nutrition and Dietetics, Sydney Children's Hospital, Sydney, Australia

Neonates with urea cycle disorders (UCD) require aggressive management. Nutritional management includes protein adequate calories and protein restriction. We present two neonates where the goals of management were to simultaneously manage hyperammonemia and hypoglycaemia. Case 1 presented on day 2 with seizures, hypoglycaemia and hyperammonemia $(2975 \mu \mathrm{mol} / \mathrm{L})$ and was diagnosed with citrullinemia. Management included hemofiltration, intravenous nutrition and ammonia-scavenging medications. Glucose intake peaked on days $1-2(29 \mathrm{mg} / \mathrm{kg} / \mathrm{min})$, requiring insulin. Ammonia decreased by day 3 to $20 \mu \mathrm{mol} / \mathrm{L}$ and it remained normal. Enteral feeding began with protein-free formula then expressed breastmilk $(\mathrm{EBM})$ at $0.5 \mathrm{~g} / \mathrm{kg}$ protein. Increasing enteral protein intake was complicated by rotavirus infection, fluid restriction and hypoglycaemia. Parenteral amino acids were added to provide $0.5 \mathrm{~g} / \mathrm{kg} / \mathrm{day}$ protein. Glucose was weaned by $1 \mathrm{mg} / \mathrm{kg} /$ day by titrating enteral feeds and IV dextrose. This increased protein by $0.1 \mathrm{~g} / \mathrm{kg} /$ day and maintained calories at $130 \mathrm{kcals} / \mathrm{kg}$. The baby was discharged growing well on oral feeds at $120 \mathrm{kcals} / \mathrm{kg}$ and $1.5 \mathrm{~g} / \mathrm{kg}$ protein. Case 2 was diagnosed with OTC deficiency after presenting with collapse and hyperammonemia $(3100 \mathrm{~mol} / \mathrm{L})$. Management was identical to that in the case above.
Protein was introduced at day 4 at $0.25 \mathrm{~g} / \mathrm{kg} /$ day from EBM in combination with protein free formula. Fluid restriction and hypoglycemia necessitated the use of parenteral amino acids when enteral feeds could not be increased. Feeds were supplemented with essential amino acids $(0.5 \mathrm{~g} / \mathrm{kg} /$ day protein) and glucose polymer. When ammonia was stable IV glucose intake was reduced by $0.5-$ $1 \mathrm{mg} / \mathrm{kg} /$ day and enteral protein intake was increased by $0.05 \mathrm{~g} / \mathrm{kg} / \mathrm{day}$ to $1.3 \mathrm{~g} / \mathrm{kg} / \mathrm{day}$. These infants presented the same challenge: to increase protein intake to prevent hyperammonemia and encourage growth, but at the same time prevent hypoglycaemia. High glucose intake in the early period likely resulted in hyperinsulinemia which was managed by weaning glucose intake.

\section{NEWBORN BABY SCREENING FOR CAH IN NEW ZEALAND}

D.Webster', P. Hofman ${ }^{2}$

LabPlus, Auckland City Hospital, Auckland, New Zealand

${ }^{2}$ Liggins Institute, University of Auckland, New Zealand

Congenital adrenal hyperplasia (CAH) is a group of disorders of cortisol biosynthesis, the most common being due to a deficiency of the 21-hydroxylase (CYP21A2) enzyme. In its more severe forms this condition easily meets common criteria for screening as it presents in the early neonatal period with adrenal insufficiency with or without salt wasting and virilisation; may not be easily clinically detected as shown by gender ratios of diagnosed patients and is easily and cheaply treated with hydrocortisone and fludrocortisone. Early detection and treatment can prevent the sudden death and damage from adrenal crises. All US states; most of Canada and 42 other countries screen newborns for CAH usually by immunoassay of 17-hydroxyprogesterone on dried blood spots. A variety of cutoffs and second tier test protocols are in use. New Zealand has been screening newborns since 1986. 1,388,409 infants have been screened for classical $\mathrm{CAH}, 61$ cases have been detected and one missed, for an incidence of 1:22,800. Screening sensitivity (20002008 ) is $100 \%$, specificity $99.7 \%$ and positive predictive value about $2.8 \%$. Followup of abnormal test results was not achieved in 6 of 530,451 infants screened 2000-2008. 25 infants were diagnosed with $\mathrm{CAH}$ in that time period; in 12 there was clinical suspicion of the condition at the time the screening test result was communicated. Those in whom there was not clinical suspicion were notified at a median of $8 \mathrm{~d}$ of age (3-16d). In addition the dried blood spot measurement of $17 \mathrm{OHP}$ is used to monitor treatment of these patients and this is especially useful for performance of diurnal studies in the home setting.

\section{COMMUNITY ATTITUDES TO GENETIC SUSCEPTIBILITY- BASED MENTAL HEALTH INTERVENTIONS FOR HEALTHY PEOPLE IN A LARGE NATIONAL SAMPLE}

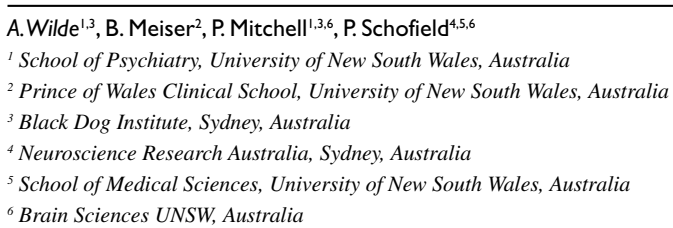

Background: Despite an apparent high interest in predictive genetic testing for common multifactorial disorders, few data describe anticipated health behavior as a consequence of such testing. T his study aimed to evaluate anticipated health behavioral responses to predictive genetic testing, using a hypothetical genetic test for risk of major depressive disorder as an example. Method: A quantitative survey of a large nationally representative population sample of 1046 Australian participants was ascertained through random digit dialing. Attitudes were assessed via structured interviews. Results: The study has identified strong community receptiveness to the use of behavioral mental health interventions in association with predictive genetic testing for major depressive disorder risk. Intention to start therapies (78\%) was significantly and positively associated with higher self-estimation of risk for depression $(p=.001)$; endorsement 
of gene-environment interaction as a causal mechanism of mental illness $(p=.017)$; and endorsing 'stressful life events' as a causal attribution for mental illness $(p<.001)$. Intention to modify potential life stressors $(84 \%)$ was significantly and positively associated with personal history of mental illness $(p=.032)$; endorsement of geneenvironment interaction as a causal mechanism of mental illness ( $p=$ $.005)$; and endorsing 'abuse' as a causal attribution for mental illness $(p<.001)$. Target groups most likely to engage in such interventions were those with a high self-reported risk of major depressive disorder and those who endorse the view that mental illness may develop from both genetic and modifiable environmental risk factors. Conclusions: Our results suggest that genetic risk information has a potential value as an early intervention and preventive tool.

\section{RISKS OF CANCERS FOR CARRIERS OF MONOALLELIC MUTYH MUTATION WITH A FAMILY HISTORY OF COLORECTAL CANCER}

A. K.Win', S. P. Cleary ${ }^{2,3}$, J. G. Dowty', N. M. Lindor', P.A. Newcomb ${ }^{5}$, R.W. Haile ${ }^{6}$, J. P.Young ${ }^{7}$, D. D. Buchanan ${ }^{7}$, L. Le Marchand ${ }^{8}$, R. Green', J. L. Hopper', S. Gallinger ${ }^{2,3}$, M.A. Jenkins'

${ }^{\prime}$ Centre for Molecular, Environmental, Genetic and Analytic Epidemiology, The University of Melbourne, Australia

${ }^{2}$ Samuel Lunenfeld Research Institute, Mount Sinai Hospital, Toronto, Canada ${ }^{3}$ Cancer Care Ontario, Toronto, Canada

${ }^{4}$ Department of Medical Genetics, Mayo Clinic, Rochester, United States ${ }^{5}$ Cancer Prevention Program, Fred Hutchinson Cancer Research Center, Seattle, United States

${ }^{6}$ Department of Preventive Medicine, University of Southern California, United States ${ }^{7}$ Familial Cancer Laboratory, Queensland Institute of Medical Research, Brisbane, Australia

${ }^{8}$ Cancer Research Center, University of Hawaii, United States

${ }^{9}$ Memorial University of Newfoundland, Canada

Background: Several studies have shown an increased risk of colorectal and extracolonic cancers for carriers of germline $M U T Y H$ mutations inherited from both parents (biallelic mutations). Extracolonic cancer risks for carriers of a MUTYH mutation inherited from only one parent (monoallelic mutation) have not previously been estimated. Materials and Methods: We identified 144 families of MUTYH mutation carriers from three countries that we ascertained through population-based sources of the multi-site, international Colon Cancer Family Registry. Mutation status, sex, age, and histories of cancer, polypectomy, and hysterectomy were sought from 2,179 of their relatives. Using Cox regression weighted to adjust for the method of ascertainment, we estimated the country-, age- and sex-specific standardized incidence ratios (SIRs) of colorectal and extracolonic cancers for monoallelic mutation carriers, compared with the general population, and corresponding age-specific cumulative risks. Results: Monoallelic mutation carriers with a family history of CRC had a significantly increased incidence of CRC (SIR 2.04; 95\% confidence interval, CI 1.56-2.70; $P<.001$ ), gastric cancer (SIR 3.24; 95\%CI 2.18-4.98; $P<.001$ ), and endometrial cancer (SIR 2.33; 95\%CI 1.18-5.08; $P$ 0.02) and a marginal increased incidence of liver cancer (SIR 3.09; 95\%CI 1.07-12.25; $P$ 0.07) compared to the general population. The estimated cumulative risks to age 70 years based on the population cancer incidence of the United States were as follows: for CRC, 6\% (95\% I 5-8\%) for men and $4 \%(95 \% \mathrm{CI} 3-6 \%)$ for women; for gastric cancer, $2 \%(95 \% \mathrm{CI}$ $1-3 \%$ ) for men and $0.7 \%$ (95\% CI $0.5-1 \%$ ) for women; for liver cancer, $1 \%(95 \%$ CI $0.3-3 \%)$ for men and $0.3 \%(95 \% \mathrm{CI} 0.1-1 \%)$ for women; and for endometrial cancer, $4 \%$ (95\%CI 2-8\%). There was no evidence of increased risks for cancer of the brain, pancreas, kidney, lung, breast or prostate. Conclusion: Monoallelic MUTYH mutation carriers with a family history of CRC are at increased risk of colorectal, gastric, endometrial and, possibly, liver cancers.

\section{LENTIVIRAL-MEDIATED CORRECTION} OF METHYLMALONIC ACIDURIA IN VIVO

E.Wong ${ }^{1,2,3}$, C. Mclntyre ${ }^{1,2,3}$, H. Peters ${ }^{4}$, E. Ranieri', ${ }^{1,2}$ D. S.Anson ${ }^{1,2,3}$, J. M. Fletcher ${ }^{1,2,3}$

${ }^{I}$ Molecular Genetics and Pathology, SA Pathology, Adelaide, Australia

${ }^{2}$ Women's and Children's Hospital, Adelaide, Australia

${ }^{3}$ Department of Paediatrics, University of Adelaide, Australia

${ }^{4}$ Murdoch Institute Royal Children's Hospital, Australia

Introduction: Methylmalonic aciduria (MMA) is a disorder of organic acid metabolism most commonly resulting from a deficiency of methylmalonyl CoA mutase. The outcome of conventional treatments MMA remains unsatisfactory and research on novel therapies remains a high priority. Positive results from liver and combined liver/kidney transplantation suggest that a metabolic sink approach to therapy may be efficacious. We have developed a lentiviral (LV) vector-mediated gene transfer treatment for an in vivo model of MMA. Aim: To examine the therapeutic effect of lentiviral vectormediated transfer of the methylmalonyl CoA mutase gene into a methylmalonyl CoA mutase knockout mouse. Methods: A self-inactivating lentiviral vector that expresses methylmalonyl CoA mutase under the transcriptional control of the elongation factor $1 \alpha$ gene promoter was constructed and injected into the tail vein of MMA mice $(n=2)$. Untreated MMA $(n=2)$ and normal mice $(n=10)$ were used as controls. Mice weight was measured weekly after treatment. Blood and urine were collected periodically to measure the level of methylmalonic acid by tandem mass spectrometry and GC/mass spectrometry, respectively. Results: Preliminary data shows that the LV-MMA treated mice began gaining weight one week after treatment and achieved a near normal weight one month later. Furthermore, the results show an approximately 5-fold and 9-fold reduction in urine and bloodspot MMA levels, respectively, in the LV-MMA treated group. Conclusion: These results show that lentiviral mediated transfer of the methylmalonyl CoA mutase gene is able to significantly reduce the metabolic abnormalities in a MMA mouse model, suggesting that the use of lentiviral vectors expressing MMA CoA mutase may be a viable approach for the treatment of this form of MMA.

\section{THE RELIABILITY AND FEASIBILITY OF NEWBORN SCREENING FOR FRAGILE X SYNDROME USING A DNA BASED FIRST TIER ASSAY}

T.Wotton',V.Wiley', B. Bennetts ${ }^{2}$, M. Field ${ }^{3}$, L. Christie ${ }^{3}$, C. Rogers ${ }^{3}$

${ }^{I}$ NSW Newborn Screening Programme, The Children's Hospital at Westmead, Australia ${ }^{2}$ Department of Molecular Genetics, The Childrens Hospital at Westmead, Australia ${ }^{3}$ The GOLD Service, Hunter Genetics, Waratah, NSW, Australia

Introduction: Each year over 100000 dried blood spot samples are analyzed by the NSW Newborn Screening Programme to provide early detection of inborn errors of metabolism, reducing morbidity and mortality. Currently DNA based testing is used as a second tier assay to screen for selected disorders including cystic fibrosis and in the future its role may expand to a first tier assay and include disorders such as fragile $\mathrm{X}$ syndrome. Aim: A pilot study has commenced in the Hunter region to assess the acceptance, feasibility and reliability of a large-scale population screening for fragile X syndrome. Samples from 2000 newborns (both males and females) are to be collected for screening after informed consent has been given. Method: A method for the detection of fragile $\mathrm{X}$ alleles in both males and females in the normal, premutation, and full mutation ranges was optimized using a PCR assay with a chimeric CGG-targeted primer (1). DNA extraction from newborn screening cards and PCR mastermix aliquoting was performed using an automated liquid handler and non-denaturing capillary electrophoresis was used for the visualisation of PCR fragments. A standard PCR -based fragile $\mathrm{X}$ assay was run in parallel to correlate with the chimeric primer assay. Results: Of the 1471 samples collected 1352 have been analyzed. Three premutations ( $>58$ CGG repeats) but no full mutations ( $>200$ CGG repeats) were found through the fragile X study. Whilst to date there have been no samples with outright PCR amplification failures, however, $4 \%$ of samples have required repeat testing. There 
has been complete concordance between the standard fragile $\mathrm{X}$ assay and the chimeric assay. Conclusion: Fragile $\mathrm{X}$ status is identifiable from the dried blood samples using the chimeric primer assay. However, the cost and time required for this assay in its current format is not suitable for routine newborn screening.

\section{DENTATORUBRAL-PALLIDOLUYSIAN ATROPHY (DRPLA) IN AN AUSTRALIAN FAMILY OF CHINESE DESCENT}

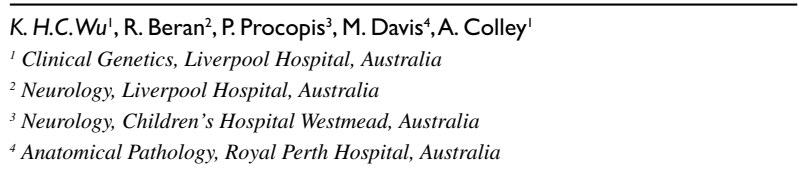

Purpose: Dentatorubral-pallidoluysian atrophy (DRPLA) is an autosomal dominant neurodegenerative disorder caused by CAG repeats in the Atrophin-1 gene. To date, three Chinese kindreds, one in Taiwan and two in Hong Kong, have been reported. We report three affected members over two generations in an Australian family of Chinese descent with DRPLA. One member was diagnosed retrospectively from stored sample. Methods: Case report on clinical data, electroencephalographic (EEG) and cerebral magnetic resonant imaging (MRI) features, and molecular genetic studies; and compare these with previously published reports. Results: The proband had migrated from China when young. He presented at age 49 yrs with progressive ataxia and cognitive decline, and became wheelchair bound in six years. His EEG showed no epileptic activity. His MRI showed generalized cerebral atrophy. His eldest son, at age $2 \mathrm{y}$ (12 years prior to proband's presentation), presented with severe mental retardation and myoclonic epilepsy, was wheelchair-bound in childhood and died at age 12 yrs. The proband's youngest daughter presented at age 7 yrs (4 years prior to the index presentation) with learning difficulties, and epilepsy onset at age 12 yrs. Her EEG showed generalized epileptiform activities. Molecular genetic studies showed CAG repeats of 58, 70, and 66 in the proband, his son, and daughter, respectively. The proband's mother is in her $80 \mathrm{~s}$ and is well. The proband's father died in South American as a young adult. Conclusion: Our family demonstrates genetic anticipation; and correlation between disease severity, age of onset and the number of triplet repeats, consistent with the literature. It however does not support the 'birth-order effect' proposed to be due to the effect of advancing paternal age on meiotic instability, as demonstrated in two other Chinese families. Further, it may be speculated that the proband's father either had a 'high-end' normal allele that subsequently expanded into pathogenic size in the proband, or had a 'low-end' pathogenic expansion and therefore did not manifest the disease prior to his death.

\section{PROBLEM FINDINGS AND USE OF OF-PCR}

\section{IN PRENATAL TESTING}

T. Saville, G. Mullan, C. Lucas, M. Caramins, M.Yip

Molecular \& Cytogenetics Unit, SEALS, Prince of Wales Hospital, Randwick (Sydney), Australia

The application of rapid aneuploidy screen as a stand-alone approach in prenatal diagnosis is much debated. Of major concern is the inability of QF-PCR testing using selected markers on only 5 chromosomes, to pick up other chromosomal abnormalities detectable on full karyotyping. This study in reviewing our cytogenetic database, seeks to evaluate the possible detection rate of abnormalities using QF-PCR testing only. A total of 5928 samples have been received for prenatal testing from 2007 to July 2010. There were 3878 amniotic fluid and 2050 chorionic villus samples. The $8.49 \%$ abnormal karyotypes were assessed in the light of a potential stand-alone QF-PCR test involving markers for chromosomes 13, 18, $21, \mathrm{X}$ and $\mathrm{Y}$. A possible $68.2 \%$ pick-up rate of abnormals was evident. The advantages of such rapid testing and also what could be missed will be highlighted. Expected concerns involved mainly cases of mosaicism, structural rearrangements and marker chromosomes. The data was also investigated for two targeted indications for referral 'advanced maternal age' and 'ultrasound abnormalities' to assess the clinical significance of chromosomal abnormalities not detectable by QF-PCR. Our laboratory commenced QF-PCR aneuploidy screen for prenatal samples in July 2008. Representative cases from the $<2.5 \%$ of inconclusive results and those discordant with karyotyping where mosaicism is an important issue will be presented.

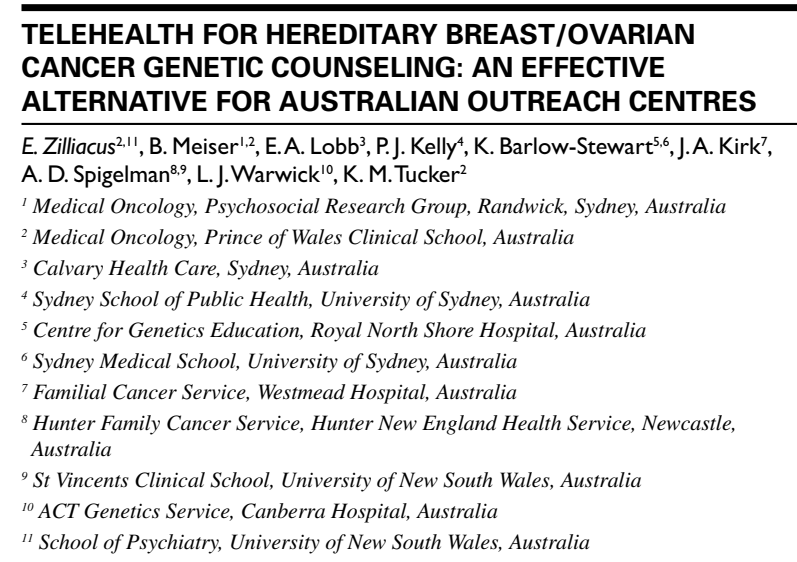

Background: Videoconferencing is increasingly used to deliver family cancer services for hereditary breast/ovarian cancer (HBOC) to outreach areas. This study used a non-inferiority design to evaluate the effectiveness of genetic counseling for HBOC through videoconferencing (hereafter referred to as 'telegenetics') compared to traditional face-to-face counseling. Methods: 106 women seen by telegenetics and 89 women seen face-to-face completed self-administered questionnaires prior to genetic counseling and one month post-consultation, measuring knowledge of HBOC, expectations, satisfaction with the genetic counseling service, perceived personal control, perceived clinician empathy, cancer-specific anxiety, generalized anxiety and depression. Results: No significant differences were found between telegenetics and face-to-face genetic counseling in terms knowledge gained $(p=.18)$, satisfaction with the genetic counseling service $(p=.48)$, cancer-specific anxiety $(p=.12)$, generalized anxiety $(p=.79)$, depression $(p=.15)$, perceived empathy of the genetic clinician $(p=.97)$ and perceived empathy of the genetic counselor $(p=.13)$. Telegenetics performed significantly better than face-to-face counseling in meeting patients' expectations $(p=.02)$, and promoting perceived personal control $(p=.02)$. Conclusion: Telegenetics performed as well as, if not better than, traditional faceto-face genetic counseling in improving patient outcomes. It appears to be an acceptable and effective method of delivering genetic counseling services for HBOC to underserved areas.

\section{'DOUBLE HIT' LYMPHOMA WITH 130 AMPLIFICATION}

A. C. Zordan, L. N. Ta, M. Curtis, A. Tejomurtula, L. J. Campbell

Cytogenetics Dept, St Vincent's Hospital Melbourne, Australia

Non-Hodgkin's lymphomas (NHLs) comprise a heterogeneous collection of malignancies and their classification has evolved steadily over the last few decades. In July 2010, a 52 year old male with newly diagnosed lymphoma had bone marrow (BM) chromosome analysis, revealing a complex karyotype including a $t(14 ; 18)$ and multiple other structural and numerical abnormalities. To clarify the rearrangements dual colour FISH, M-FISH and m-BAND were applied. Conventional FISH using a $M Y C$ break apart probe revealed an insertion of 3' $M Y C$ into proximal $2 p$ suggestive of a cryptic $\mathrm{t}(2 ; 8)(\mathrm{p} 12 ; \mathrm{q} 24)$. M-FISH confirmed the $\mathrm{t}(14 ; 18)$ and also revealed rearrangements and insertions of $13 q$, which with the aid of $m$ BAND proved to be amplification of the 13q31-q32 region. Although immunoglobulin gene/MYC translocations are the hallmark of Burkitt lymphoma, they are also found in a subset of DLBCL $(\sim 5-10 \%)$. Furthermore, the $\mathrm{t}(14 ; 18)$ translocation identified in up to $90 \%$ of Follicular Lymphomas has also been reported in $10 \%$ to $40 \%$ of DLBCL. It has been reported that approximately 
$24 \%$ of B-cell lymphomas with $M Y C$ rearrangements shown 13q31 amplification. In NHL, a $1.4 \mathrm{Mb}$ amplicon of 13q31-q32 has been reported in DLBCL of the germinal centre B-cell like (GCB) DLBCL subtype. The gene in this region that is most over expressed in DLBCL has been shown to be MIRI7HG which encodes the mir17-92 polycistronic cluster. The miRNAs encoded by the mir-17-92 polycistron are known to act as oncogenes with their expression promoting cell proliferation, suppressing apoptosis and inducing tumour angiogenesis. The findings of an $I G H / B C L 2$ and a possible $I G K / M Y C$ translocation in our patient indicate that this patient falls into the "double hit" category of DLBCL that has been reported previously to be associated with an extremely poor prognosis. Moreover, 13q31-q32 amplification has been observed predominantly in lymphomas containing $M Y C$ rearrangements, supporting the suggestion that $M Y C$ and $M I R I 7 H G$ may act synergistically in aggressive lymphomas.

\section{TELOMERIC LOSS AND BREAKAGE-FUSION-BRIDGE CYCLES: A MECHANISM FOR GENETIC AMPLIFICATION, RING FORMATION AND DICENTRIC CHROMOSOME DEVELOPMENT LEADING TO INHERENT GENOMIC INSTABILITY.}

L. N. Ta, A. C. Zordan, R. MacKinnon, B. Mercer, L. J. Campbell

Cytogenetics Dept, St Vincent's Hospital Melbourne, Australia

Telomeres play an important role in chromosome stability by preventing degradation and fusion of chromosome ends. The loss of a single telomere may result in chromosomal instability through breakagefusion-bridge (BFB) cycles. In this process the chromosome with the lost telomere is replicated and the ends of the resultant sister chromatids fuse together. A bridge is therefore formed which often breaks near the centre during anaphase as the two chromatids are pulled to opposite poles. The chromosome is then replicated and once again the cycle begins. This mechanism produces gene amplification as well as ring and dicentric chromosome formation. The BFB cycle continues until a new telomere is acquired, frequently through translocation of the unstable chromosome to ends of other chromosomes. A 72-year-old female with CMML-2 referred for bone marrow chromosomal analysis provides an illustration of the BFB cycle leading to gene amplification. Conventional cytogenetics revealed a complex hypodiploid karyotype with abnormalities of chromosomes 5 and 7 and ring and dicentric chromosomes. Subsequent MFISH testing revealed that a $\operatorname{der}(11) \mathrm{r}(11: ?)$ was composed entirely of chromosome 11 material and that the $\operatorname{der}(12 ; 11 ; ?)$ was composed of chromosomes 11, 12 and 15. Further FISH testing using a dual colour MLL break-apart probe showed five copies of MLL on both the der(11)r(11:?) and der(12;11;?) chromosomes. The results of the investigation revealed clonal evolution involving gene amplification, and the formation of dicentric and ring chromosomes. The initial cell line contained two normal chromosomes 11 and a copy of the dic(12;15). As the disease progressed, a ring 11 was formed. Finally, in order to gain telomeres and thus stability, it appears that the ring 11 unwound and inserted itself into the dic $(12 ; 15)$ chromosome forming a tricentric chromosome which may yet lead to further instability and disease progression. This investigation shows that BFB cycles play an important role in chromosomal rearrangements and clonal evolution in malignant cells. 